UC-22

Issued: July 1982

$L \lambda--9420-T$

DEN2 021844

\title{
Rapid Vibrational and Rotational Energy-Transfer Rates in Heated Carbon Dioxide Collisions by Double-Resonance Laser Spectroscopy
}

Michael David Thomason

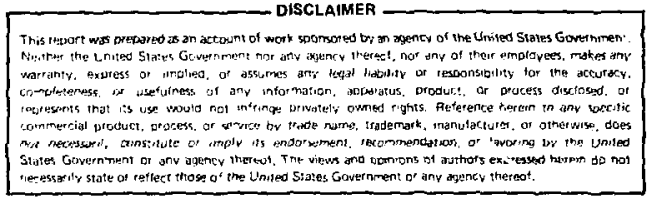

Los

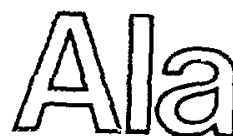

กS Los Alamos National Laboratory Los Alamos,New Mexico 87545 
CONTENTS

Figures. . . . . . . . . . . . . . . . vi Tables............................ vij Abstract..................... 1 Chapter I - Introduction ............ 2 Chapter II - Relevant Background Material . . . . 12

A. Molecular Structure of $\mathrm{CO}_{2}$. . . . . . . 12

B. Energy Transfer Cross Sections ...... 26

C. Rate Constants and Rate Equations .... 37

D. Double Resonance Spectroscopy.......44 Chapter III - Relaxation Model Studies... . . . 54

A. Rotation Transfer Models ........ 55

1. The Reservoir Model . . . . . . 57

2. The $\Delta J=2$ Model ......... 62

3. The Ground State V-V Model ..... . 64

4. The He+CO Model . . . . . . . 68

5. Discussion of the R-R Models.... . 69

B. Resonant $v_{3}$ Transfer Studies . . . . . 71

1. The Multilevel Computer Model . . . 71

2. The Three-Level Model ....... 75 
3. The $n_{002}$ Solution ....... 82

4. Sequence-Band Study ....... 84

Chapter IV - Experimental Apparatus and Procedure . 89

A. Experimental Apparatus ....... 89

B. Signar Detection ........... 95

C. The $\mathrm{CO}_{2}$ Lasers .......... 100

D. Experimental Procedures ......... 107

Chapter V - Analysis and Results .........121

A. Rotational Energy Transfer Results. .... 121

1. Pumped Level Relaxation . . . . . 122

2. J-Dependence of the Relaxation ....123

B. Rapid Vitrational Energy Transfer..... 129

1. The $v_{3}$ Transfer Results ...... 133

2. The $v_{2}$ Transfer Hypothesis ..... 142

Chapter VI - Discussion and Final Remarks . . . . 147

A. Rotational Energy Transfer ....... 147

B. Resonant Vibrational Energy Transfer ... 150

c. Final Remarks.......... 155

Acknowledgments. . . . . . . . . . . 159

Bibliography................... 160 


\section{FIGURES}

II-1. Vibrational Energy Levels of $\mathrm{CO}_{2} \cdot . \cdot . \cdot . \cdot 13$

II-2. Rotational 0ccupation Probability at $700^{\circ} \mathrm{K}$. 20

I]-3. Probe Transmission vs Time........ 51

III-2. The Pumped Level Response at 4 Torr . . . 60

III-3. The J-Dependence of the Relaxation at 4 Torr. 61

III-1. The $\Delta J=2$ Rate Constant Matrix ...... . 63

III-4. The Three-Level Model Response....... 76

III-5. Response of the Sequence Levels . . . . 86

III-6. The Sequence-Band Study ........ . 88

IV-1. The Doutle Resonance System . . . . . . 90

IV-2. Electrical Diagram of the Shutter . . . . 93

IV-3. Screen-Room Optics and Electronics..... 96

IV-4. Laser Timing and Firing Diagram . . . . . 106

IV-5. Observed Decay of the Pumped Level ......110

IV-6. Samples of j-Dependent Relaxation . . . . 113

IV-7. Hot-Band Probe Data ........... 114

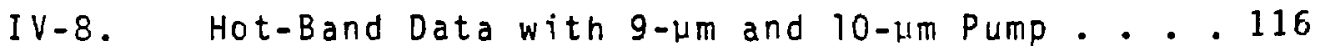

IV-9. Fast Sweep Sequence-Band Data at 10 Torr . . 118

IV-10. Sequence-Band Data with Slower Sweep ... 119

$V-1$. Sample Plot of $T^{-1}$ vs Pressure....... 124

V-2. Long-Term J-Dependence of Rotational

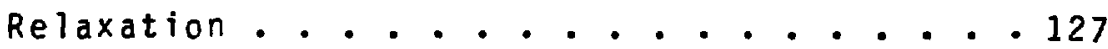


V-3. Long-term J-Dependence of Rotational Relaxation . . . . . . . . . . 128

V-4. Short-Term J-Dependence of Rotational

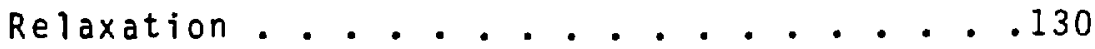

V-5. Short-Term J-Dependence of Rotational

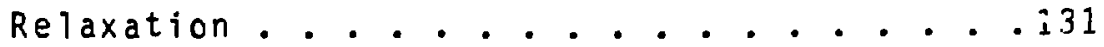

V-6. Risetimes of the $\Delta \mathrm{J}$ Responses . . . . . . 132

V-7. Hot-Band Data and Fit .......... 136

V-8. Slow-Sweep Sequence-Band Data and Fit . . . 137

$V-9$. Fast-Sweep Sequence-Band Data and Fit . . 138

V-10. Plot of the Final $n_{017^{-n}}$ 110 Model . . . . 146

TABLES

II- 1 CO2 Energy States and Populations at Four Torr .............. 21

Il-2 Selected $\mathrm{CO}_{2}$ Infrared Transitions . . . . 23

$V-1$ Resulis for Pumped Level Rotational

Relaxation ..................... 125

V-2 Least-Squares Fit Information for $v_{3}$

Transfer.............. 141

VI- $]$ Results of $v_{3}$ Transfer Measurements

and Comparison to Theory........ 152 
RAPID VIBRATIONAL AND ROTATIONAL ENERGY TRANSFER RATES IN HEATED CARBON DIOXIDE COLLISIONS

BY DOUBLE RESONANCE LASER SPECTROSCOPY

by

Michael David Thomason

ABSTRACT

Rates for resonant vibrational and rotational energy transfer from the 001 state by $\mathrm{CO}_{2}+\mathrm{CO}_{2}$ collisions have been measured. All data were obtained by double resonance spectroscopy with $\mathrm{CO}_{2}$ lasers in a 2.5 meter absorption cell at $700^{\circ} \mathrm{K}$. Results for rotation transfer include pumped-level relaxation and the response of other 001 levels with $\Delta J$ up to 18 . These data are compared to four relevant collision models via a $35-1$ evel rate equation analysis.

Sequence-band $(002+101)$ and hot-band $(011+110)$

lasing have been used to observe resonant $v_{3}$-transfer relaxation involving $001+001 \leftrightarrow 002+000,001+100 \leftrightarrow 101+000$, and $00 !+010 \leftrightarrow 011+000$. A multilevel rate analys is has been utilized to determine the rate coefficients for 001 going to the 002, the 101, and the 011 levels. Part of the hot-band data has been interpreted as due to $110+000+100+010$, and the associated rate constant has been estimated.

The results of the study are compared to the theory and to other experiments. 
CHAPTER I

INTRODUCTION

In the following chapters I have described my experimental investigation of collisional energy transfer from the 001 state of $\mathrm{CO}_{2}$, which is the upper laser level of the $\mathrm{CO}_{2}$ laser. The processes studied are rotational energy transfer and resonant viorational energy transfer. These reactions are responsible for the fastest population transfer between states that occurs when the 001 population changes. These respoases can readily be observed by transmission changes of laser frequencies involving the energy levels of interest. This is the basis of the technique of double resonance spectroscopy, which has been my primary investigative tool.

The motivation for many studies of $\mathrm{CO}_{2}$ in the recent past has been to improve the understanding of the $\mathrm{CO}_{2}$ laser. This is true in this work also. In addition, $\mathrm{CO}_{2}$ is an ideal candidate for detailed study of energy transfer in polyatomic molecules.

Polyatomics display two very interesting characteristics which cannot be studied in diatomic experiments. The first is that transition dipole moments can occur without the requirement that the molecule have a permanent dipole moment. Secondly, collisions can affect 
polyatomics in such a way that the different vibrational modes undergo energy relaxation at different rates. Both effects are observed in $\mathrm{CO}_{2}$. The first is believed to cause the very large $v_{3}$-vibration exchange cross sections. The second is a major factor in the high efficiencies which have been achieved in $\mathrm{CO}_{2}$ lasers.

Polyatomic collisions are generally difficult to understand on a state-to-state basis because even simple molecules have complicated level structure compared to diatomics. $\mathrm{CO}_{2}$ has only three atoms, is linear, and is symmetric. This results in a relatively simple and well understood system of energy levels. The spectroscopy of these levels is well studied also, and data needed for collision work and laser work are readily available in the literature.

The laser has become an important tool in molecular collision physics. The $\mathrm{CO}_{2}$ laser is an especially unique tool for study of $\mathrm{CO}_{2}$ collisions as it allows state-by-state observations in double resonance spectroscopy. Given this and the dozens of transitions available, it should eventually be possible to completely understand the energy pathways in $\mathrm{CO}_{2}$ and the energy transfer cross sections on a state-to-state basis.

Theoretical descriptions are not well developed for polyatomic collisions at this time. However, they are 
beginning to evolve. Experiments of this sort should be able to really test the details of collision theory as it is extended into the realm of collisions of simple polyatomics.

Besides the basic interest in polyatomic collision physics described above, my measurements should have practical use as data for $\mathrm{CO}_{2}$ laser modeling. The conventional multitemperature models of these lasers has required only crude rate coefficients to successfully predict output properties. However, there is increasing interest in laser medium repumping after pulsed energy extraction. Understanding this process seems to require more data than is presently available. It is in this area that my results may be valuable.

Much of the previous relevant work on rotation and vibration transfer, which I will now describe, has been directed to the needs of 1 aser modeling. These experiments have also made an important contribution to $\mathrm{CO}_{2}$ collision physics. In lany cases, however, I have found the depth of the investigations insufficient to completely understand the collision mechanisms which were involved.

There are three previous rotational relaxation experiments of interest (Carrol and Marcus, 68) :Cheo and Abrams, 69) (Jacobs et al., 74a, b). These experiments 
have many similarities. Each is a double resonance experiment. Also, the $\mathrm{CO}_{2}$ being investigated is contained in an electrical discharge which gives the sample some gain. In some instances $\mathrm{He}$ and $\mathrm{N}_{2}$ are present in the medium. Each experiment relies on an intense, short, external laser pulse to deplete the steady state 001 population. Population transients on a variety of rotation levels are monitored by changes in transmission observed on a second laser whose path overlaps the first in the amplifier cell.

If it is assumed that for short times rotational exchange is the dominant collision process, the 001 population should change in the following way. The J-level associated with the strong pulse is severely depleted. Subsequent collisions cause this level to regain population at the expense of the other 001 levels. All the levels attain rotational equilibrium again with some overall loss of 001 population. This is observed in all these experiments. The interesting features are the recovery time of the depleted level and the character of the response of the other $001 \mathrm{~J}$-levels. These are of course governed by the state-to-state rotational energytransfer cross sections.

The depleted level recovery time was measured in a direct manner by the Jacobs and the Cheo experiments. By 
probing the depleted level with an 9-um transition while

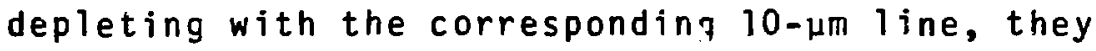
directly monitored the populatioli recoyery. By fitting a time constant to the exponential oehavior of the recovery, thes were able to derive an effective rotatiunal rate constant. Tr.e results for pure $\mathrm{CO}_{2}$ are $1.1 \pm 0.3 \times 10^{7}$ (Cheo and Abrams, 69) and $1.3 \pm 0.2 \times 10^{7}$ (Jacobs et a 1., $74 a)$ in units of $\sec ^{-1}$ torr $^{-1}$. These agree within the quoted errors.

The experiment of Carrol and Marcus has the distinction of being the first performed, but their result is not in agreement with the other two. Because they used an R-branch pulse and P-branch monitor, both of the 10-um band, it is somewhat difficult to tell whether their signals relate primarily to the 001 level or the 100 . Their detailed rate equation analysis yielded an effective $\mathrm{CO}_{2}$ rate of $2.0 \pm 0.3 \times 10^{-10} \mathrm{~cm}^{3} \mathrm{sec}^{-1}$ at $350^{\circ} \mathrm{K}$ or $5.5 \pm 0.8 \times 10^{6} \mathrm{sec}^{-1} \mathrm{torr}^{-1}$. However, their state-to-state rate coefficient matrix assumed only $\Delta \mathrm{J}=2$ elements were allowed. Their result, of course, reflects the use of this collisional selection rule.

Each of the three experiments also monitored the behavior of the neighboring J-levels. As stated before Carrol and Marcus reported evidence of $\Delta J=2$ selection rules. The preference tir small $\Delta J$ changes in 
collisions will be seen in data as non-monotonic equilibration, especially in the nearest neighbor levels. This behavior is evident in their published data. A monotonic return tu equilibrium for all levels would imply $\Delta \mathrm{J}$-independent rotation changes. The Cheo and Abrams work found all the neighboring level responses to be very similar and apparently monotonic. Thus, they concluded the rates must be $\Delta J$-independent. Jacobs et al. later found the observed responses, with their conditions, were not monotonic. However, when they analyzed their results with a $\Delta J=2$ rate model, they were not able to reproduce the leading edge behavior of their response data. They stated larger $\Delta J$ exchange would also be necessary.

Some $\Delta \mathrm{J}$ dependence seems to be indicated except in the Cheo and Abrams case. This happens to be the only one of the experiments in which the sample was pure $\mathrm{CO}_{2}$ during the neighboring line measurements.

An interesting interpretation (Sharma and Kern, 71), (Moore, 73) of the pure $\mathrm{CO}_{2}$ results involves the $\mathrm{v}_{3}$ resonant $V-V$ reaction between molecules $A$ and $B$.

$$
\left(001, J_{A}\right)_{A}+\left(000, J_{B}\right)_{B} \leftrightarrow\left(000, J_{A} \pm 1\right)_{A}+\left(001, J_{B} \pm 1\right)_{B}
$$

As will be discussed below this is a very fast process although probably not as rapid as the gas kinetic rate. 
Sharma and Kern state that, if this were the dominant mechanism for rotational relaxation of the 001 level, monotonic response would result. This process is mentioned in the Jacobs et a 1. paper but is not included in their analysis. Sharma and Kern also mention that the $\Delta J=2$ mechanism should be important, but they do not elaborate on its effects.

The Jacobs results have been explained very well (Preston and Pack, 78) in terms of He and $\mathrm{CO}_{2}$ collisions. Some adjustments of this model were necessary, but the non-monotonic features and risetimes were clearly reproduced.

It would seem that the above affords a reasonable explanation of the observations. That is, that rotationa? relaxation in pure $\mathrm{CO}_{2}$ is governed by rotational changes associated with $V-V$ transfer while relaxation in mixtures is due to pure rotational exchange as in the Pack model. My study will attempt to determine which mechanisms are important in pure $\mathrm{CO}_{2}$ rotational relaxation.

vibrational relaxation generally occurs on a much slower time scale than rotational relaxation. There are, however, some $V-V$ processes in $\mathrm{CO}_{2}$ that are rapid enough to compete with the rotation transfer. These are the so called resonant vibrational exchange collisions in which the total internal energy change is less than about 
$50 \mathrm{~cm}^{-1}$. Collisions of this type profoundly affect 001 behavior. My primary interest is in this type of $V-V$ mechanism in this study.

Soon after it was suggested (Mahan, 67) that vibrational transition dipole moments of colliding molecules could interact with a long range $R^{-3}$ potential, calculations were carried out for many cases (Sharma and Brau, 67, 69). The early work utilized impact parameter formulations. Recertiy OWBA (Distorted-Wave Born Approximation) has been applied to $v_{3}$ exchange in $\mathrm{CO}_{2}$ self collisions (Pack, 80).

The salient feature of this type of resonant interaction is a large cross section which varies as $T^{-1}$ (Moore, 73). Experiments are usually in good agreement with the theory in magnitude and temperature dependence (Stephenson and Moore, 72). It is true, however, that as collisions become less resonant (>50 $\mathrm{cm}^{-1}$ ) the theory underestimates the observed cross sections (Pack, 80).

Three experiments have been performed which measured fast $v_{3}$ exchange processes in $\mathrm{CO}_{2}$. Two of these (Burak et a 1., 73) (Doyennette et al., 75) interpreted their observations as due to

$$
(001)+(010) \rightarrow(011)+(000)
$$

The other (Finzi and Moore, 75) looked at the two reactions: -

$$
\begin{aligned}
& (001)+(100) \leftrightarrow(101)+(000) \\
& (001)+(020) \leftrightarrow(021)+(000)
\end{aligned}
$$


These are all laser induced fluorescence measurements. The agreement with theory is good (Pack, 80) although some discrepancies are apparent with the very good data of the Doyennette et al. work.

One objective of my experiment is the determination of the rate coefficient for

$$
(001)+(001) \leftrightarrow(002)+(000)
$$

This process is expected to have an extremely large cross section (Pack, 80). With this rate and those mentioned above, an accurate understanding of the role played by $v_{3}$ exchange in $\mathrm{CO}_{2}$ laser processes should be possible.

The state of knowledge of fast 001 relaxation, summarized above, indicates a need for further work. My work was initiated with the intent of producing an in-depth study which would significantly contribute to the elimination of this need.

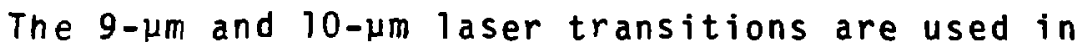
this work for rotational exchange, and measurements are performed in much the same manner as the cheo and the Jacobs experiments.

However, in my experimental setup the double resonance occurs in a heated absorption cell instead of an amplifier. In aodition to better control of pressure and impurities, this arrangement allows one to know exactly 
the population distribution of the various energy levels. This is imperative if a detailed analysis is to be accomplished. All of the measurements to be described have been performed at a temperature of $700^{\circ} \mathrm{K}$.

$\mathrm{CO}_{2}$ also lases, with a little additional effort, on the hot-band $(011+110)$ and the sequence-band $(002 \rightarrow 101)$ transitions. I have used these frequencies as probe beams to determine rate constants for the reactions

$$
\begin{aligned}
& (001)+(001) \leftrightarrow(002)+(000) \\
& (001)+(100) \leftrightarrow(101)+(000) \\
& (001)+(010) \leftrightarrow(011)+(000)
\end{aligned}
$$

Before the details of the experimental work are given, Chapters II and III, respectively, discuss background material and rate model studies. The model studies are immensely helpful in interpreting the rather complicated double-resonance data. The last three chapters, then, address the experiment and the results. 
CHAPTER II

\section{RELEVANT BACKGROUND MATERIAL}

A. Molecular Structure of $\mathrm{CO}_{2}$ (Herzberg, 45)

Carbon dioxide is a triatomic molecule which is linear and symmetric. The total wavefunction is approximately the product of the electronic, vibration, and rotation wavefunctions.

$$
\psi=\psi_{e} \psi_{v} \psi_{r}
$$

The energy level diagram of $\mathrm{Fig}$. II-l shows many important features of the vibrational structure. All the vibration levels through $2349 \mathrm{~cm}^{-1}$ are indicated. The number of levels increases rapidly at higher energies, so only those states of immediate interest are shown in that region. The fundamental vibration modes $\left(v_{1}, v_{2}\right.$, and $v_{3}$ ) are the symmetric stretch, the bending, and the asymmetric stretch vibrations. The classical analoque of each of these is illustrated below the corresponding mode symbol in the figures. The arrows indicate the restoring force directions. The symbol $v_{i}+v_{j}$ represents a combination mode.

The vibrations behave like normal-coordinate harmonic oscillators to good first approximation. The number of 


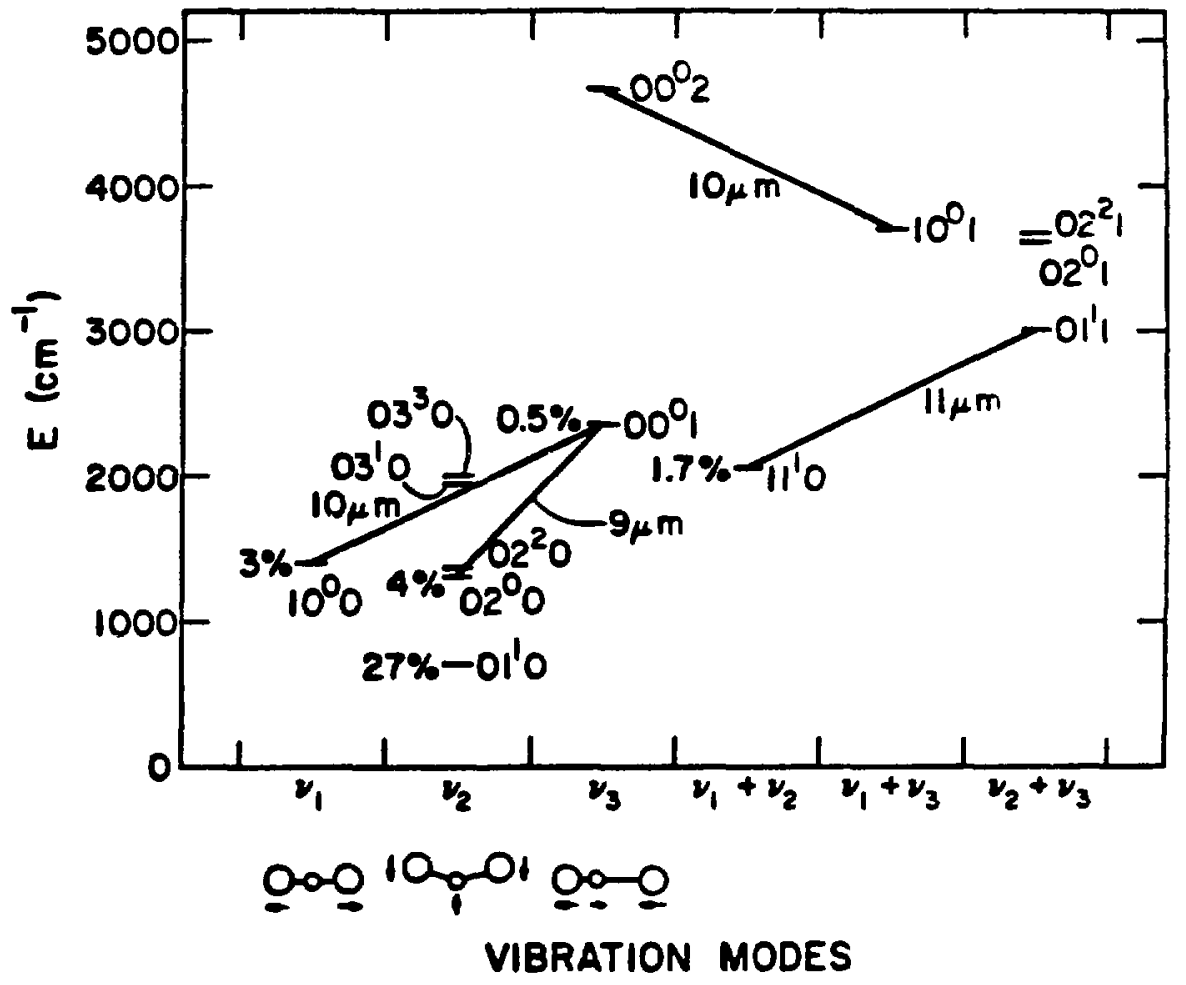

Figure II-1. Vibrational Energy Levels of $\mathrm{CO}_{2}$

All levels through 001 at $2349 \mathrm{~cm}^{-1}$ are shown along with the higher levels which are important to the study. Also shown are the relevant laser transitions and some occupation probabilities at $700^{\circ} \mathrm{K}$. 
quanta in each mode is signified by $v_{1}, v_{2}$, and $v_{3}$ for the respective modes. The $v_{2}$ mode is the most complicated; its behavior is like a two-dimensional oscillator. It can have angular momentum along the axis. The associated quantum number of this rotation is $\ell$, which can range up to the value of $v_{2}$ and must be even or odd as $v_{2}$ is, thus

$$
\begin{aligned}
& \ell=0,2, \ldots, v_{2}\left(v_{2} \text { even }\right) \\
& \ell=1,3, \ldots, v_{2}\left(v_{2} \text { odd }\right)
\end{aligned}
$$

The angular momentum vector can be in either axis direction and $l \neq 0$ levels consist of two degenerate states, one for each angular momentum.

The traditional level notation is

$$
\left(v_{1} v_{2}^{\ell} v_{3}\right)
$$

If two levels are close in energy and have the same $\ell$ value, anharmonic perturbations can cause strong coupling of the states with accompanying energy shifts. This phenomenon is called Fermi degeneracy, or mixing, after its discoverer. The levels $02^{\circ} \mathrm{O}$ and $10^{\circ} \mathrm{O}$ are good examples. They are almost eveniy mixed states of the $v_{1}$ and $v_{2}$ modes. 
The rotation with angular momentum vector normal to the axis is similar to diatomic rigid-rotor motion with wavefunctions being spherical harmonics. Superimposed on each vibrational energy level, then, is the rotational energy structure with

$$
E_{R O T}\left(\mathrm{~cm}^{-1}\right)=B J(J+1)-D J^{2}(J+1)^{2} \quad I I-2
$$

with $\mathrm{J}$ equal to the total angular momentum. For $\mathrm{CO}_{2}$ $B=.39 \mathrm{~cm}^{-1}$. The term involving $D$ is small and is usually not important in collision work.

The electronic angular momentum about the axis is denoted by quantum number $A=0,1,2, \ldots$, and the corresponding state is designated by $\Sigma, \pi, \Delta, \ldots$, respectively.

The symmetry properties of $\mathrm{CO}_{2}$ are complicated, but a few rules and definitions seem to determine many of the characteristics of the states and the spectroscopy.

The parity transformation, or inversion through the center of the molecule, has quantum number \pm 1 depending on whether or not the total eigenfunction changes sign when the coordinates of all nuclei and electrons change sign. The parity of the electrons is denoted by 9 (gerade) or $u$ (ungerade). The $g$ is a +1 , or even state, while the u implies -1 . For the vibration wavefunction, the parity is determined by summing the $v_{2}$ and $v_{3}$ quanta; the result is $(-1)^{v_{2}+v_{3}}$. The 
spherical harmonics $Y_{J M}$ are even or odd functions as determined by $(-1)^{3}$.

A state is designated by its species symbol. For example the ground state $00^{\circ} 0$ is $\Sigma_{g}^{+}$. Here, $\Lambda=0$, even electronic parity, and even total parity are indicated.

In $\mathrm{CO}_{2}$ the $0^{16}$ nuclei are $\mathrm{spin}=0$ bosons, so interchange of their coorainates must leave the sign of the total wavefunction unchanged. This is called exchange symmetry and each possible energy level is denoted by $s$ or a to show whether it is exchange symmetric or antisymmetric, respectively. An important consequence of this is alternate $J$ levels are missing in all $l=0$ states. This results because the behavior under exchange is determined by the symmetry of the product $\psi_{v} \psi_{r}$, thus $(-1)^{v_{2}+v_{3}+J}$ must be even. As a consequence, the $00^{\circ} 0,02^{\circ} 0,10^{\circ} 0,00^{\circ} 2$ states have only even $\mathrm{J}$ while $00^{\circ} 1,02^{\circ} 1,10^{\circ} 1$ have only odd. When $\ell \neq 0$ the exchange does not constrain the $J$ values because for each $J$ there is a positive and a negative level of slightly different energy due to the two $l$ states (Herzberg, 45, p. 372). So even though the symmetry does apply, all J levels are present, but one of the \pm levels for each J doesn't exist. 
It is also true that all electronic ground states of $\mathrm{CO}_{2}$ have positive total parity, as determined by $\psi_{e} \psi_{y}$. Thus for $(-1) v_{2}+v_{3}=+1, g$ is required while -1 implies $u$. Examples are $000\left(\Sigma_{g}^{+}\right)$ and $001\left(\Sigma_{u}^{+}\right)$. This rule also dictates that 011 is $I_{g}$ which is not 1 isted ir Herzberg because there is no ground state absorption. The state is important, however, as it is the upper level of the hot-band $(011+110)$ laser transition.

In a gas sample at laboratory temperatures $\left(700^{\circ} \mathrm{K}\right.$ in the present casel, many rotational levels are occupied, and significant population density can also appear in vibrational states above the ground state. Calculation of the populations requires the value of the partition function at temperature $T$,

$$
Q=\sum_{n} g_{n} e^{-E_{n} / k_{B} T}
$$

in which $E_{n}$ is the energy of state $n, g_{n}$ is the degeneracy and $k_{B}$ is Boltzmann's constant.

For rotational levels, the population density $\left(\mathrm{cm}^{-3}\right)$ is

$$
N_{J}=\frac{N_{v}}{Q_{r}} g_{J} e^{-E_{J} / k_{B} T}
$$

in which $Q_{r}$ is the rotation partition function and

$$
g_{J}=2 J+1
$$


In the above, $N_{v}$ is the total population of a given vibrational level,

$$
N_{v}=\frac{N_{G}}{Q_{V}} g_{v} e^{-E_{v} / k_{B} T}
$$

Here $Q_{V}$ is the vibrational partition function, $N_{G}$ is the gas density and

$$
\text { I for } \ell=0
$$

$$
g_{v}=
$$

$$
2 \text { for } \ell \neq 0
$$

There are approximate expressions derivable for $Q_{v}$ and $Q_{r}$. For rotation

$$
Q_{r}=\frac{k_{B} T}{\sigma h C B}
$$

with $\sigma=1$, for $\ell \neq 0$, or 2 for $\ell=0$. For vibration

$$
\begin{gathered}
Q_{v}=\left[1-\exp \left(-v_{1} h v_{1} / k_{B} T\right)\right]^{-1}\left[1-\exp \left(-v_{2} h v_{2} / k_{B} T\right)\right]^{-2} \\
\times\left[1-\exp \left(-v_{3} h v_{3} / k_{B} T\right)\right]^{-1}
\end{gathered}
$$

in which the vibrational mode quantities are implied. When detailed computer calculations are made, it is more convenient to perform the sums directly. Of course, one must include in the sum all levels which have nonnegligible population. 
Fig. II-2 shows the $700^{\circ} \mathrm{K}$ rotational occupation probability,

$$
P_{J}=\frac{2 J+1}{Q_{r}} e^{-E_{J} / k_{B} T}
$$

which is appropriate for a vibration with odd $J$ levels. Shown also are the level energies. Table II-l shows vibrational state energies, degeneracies, and probabilities through level 001 . Also given are the population densities calculated for 4 torr. A few percent-occupation values are also indicated in Fig. II-1.

The infrared spectroscopy of $\mathrm{CO}_{2}$ results from the rotation-vibration transitions and dipole radiation laws. Transition strengths are governed by the dipole matrix element, $|\langle f|\mu| i\rangle|$, which is called the transition dipole moment, $\mu$. The transitions obey the selection rules which govern rotational and vibrational transitions separately (Herzberg, 45, p. 379), that is,

$$
\Delta l=0, \pm 1 \quad \Sigma^{+}+/+\Sigma^{-} g+/ \rightarrow g u+/+u \text { (vibration) }
$$

$$
\Delta J=0, \pm 1 \quad(J=0+/+J=0)+\leftrightarrow-s+/+a \text { (rotation) }
$$

Harmonic oscillator selection rules $v+v \pm 1$ are not effective because oi state mixing and the nonlinearity of u. However, line strengths are influenced by them. 


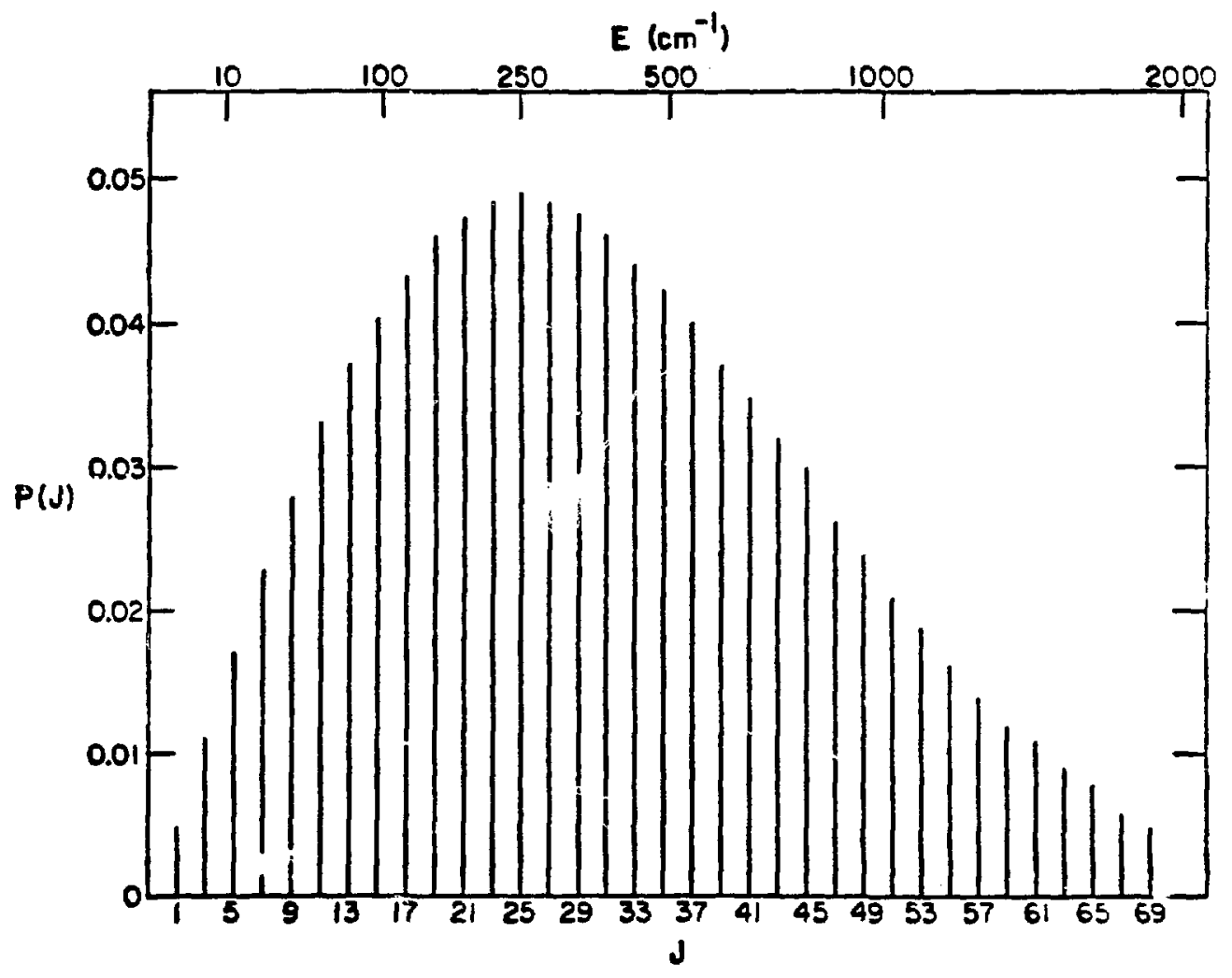

Figure II-2. Rotational Occupation Probability at $700^{\circ} \mathrm{K}$

The probabilities shown are those appropriate for a level with only odd $J$ values such as 001 . 
TABLE II-1

State $E_{v}\left(\mathrm{~cm}^{-1}\right) \quad g_{V} \quad P_{V} \quad N_{v}\left(\times 10^{16}\right)$

$\begin{array}{lrlll}00^{\circ} 0 & 0 & 1 & .516 & 2.750 \\ 01^{1} 0 & 667 & 2 & .275 & 1.464 \\ 02^{\circ} 0 & 1285 & 1 & .040 & .215 \\ 02^{2} 0 & 1335 & 2 & .073 & .389 \\ 10^{\circ} 0 & 1388 & 1 & .033 & .175 \\ 03^{1} 0 & 1932 & 2 & .022 & .119 \\ 03^{3} 0 & 2003 & 2 & .019 & .103 \\ 11^{1} 0 & 2077 & 2 & .017 & .089 \\ 00^{\circ} 1 & 2349 & 1 & .005 & .026\end{array}$


Table II-2 gives some pertinent information for selected $\mathrm{CO}_{2}$ transitions. The states involved and the associated species symbols are given in the first and second columns. The third column shows the radiation, or spontaneous emission, rate, $A\left(\sec ^{-1}\right)$. The values shown are the average of the $\Delta J=+1$ and $\Delta J=-1$ values previously calculated (Statz et al., 66). These lifetimes are very long compared to collisional relaxation times even at 1 torr, so radiation isn't usually an important relaxation mechanism. In the fourth column are representative wavelengths determined from subtraction of the vibrational levels. A wavelength is needed to calculate a transition moment from A (Schiff, 69, p. 414) by the relation,

$$
\mu^{2}=(3 / 4)(h / 2 \pi)(\lambda / 2 \pi)^{3} A
$$

which is shown in the last column. The unit given, $10^{-18}$ esu (statcoulomb-cm), is often called a Debye.

The transition dipole is a rather intuitive quantity that can be physically understood as a charge separation of atomic dimensions which is oscillating in time. The harmonic-oscillator relationship between transition dipoles,

$$
\mu(v \leftrightarrow v-1)=\mu(1 \leftrightarrow 0) \times(v)^{\frac{3}{2}}
$$

holds very well even though the selection rules don't, as can be seen, from the table, by comparing transitions 1) 
TABLE II -2

\begin{tabular}{|c|c|c|c|c|c|}
\hline \multicolumn{2}{|c|}{ Transition } & \multirow{2}{*}{$\frac{\text { Species }}{\Sigma_{u}^{+}-\Sigma_{g}^{+}}$} & \multirow{2}{*}{$\frac{A\left(\sec ^{-1}\right)}{205}$} & \multirow{2}{*}{$\frac{\lambda_{0}(\mu \mathrm{m})}{4.26}$} & \multirow{2}{*}{$\frac{\tau_{\mu\left(10^{-18} \text { esu }\right)}}{0.224}$} \\
\hline 1) & $00^{\circ} 1-00^{\circ} 0$ & & & & \\
\hline 2) & $00^{\circ} 2-00^{\circ} 1$ & $\Sigma_{g}^{+}-\Sigma_{u}^{+}$ & 400 & 4.30 & 0.318 \\
\hline 3) & $00^{\circ} 1-10^{\circ} 0$ & $\Sigma_{u}^{+}-\Sigma_{g}^{+}$ & .335 & 10.4 & 0.035 \\
\hline 4) & $00^{\circ} 1-02^{\circ} 0$ & $\Sigma_{u}^{+}-\Sigma_{g}^{+}$ & .195 & 9.40 & 0.023 \\
\hline 5) & $08^{1} 0-00^{\circ} 0$ & $\pi_{u}-\Sigma_{g}^{+}$ & .470 & 15.0 & 0.071 \\
\hline 6) & $02^{2} 0-01^{1} 0$ & $\Delta_{g}-\Pi u$ & .950 & 15.0 & 0.101 \\
\hline 7) & $02^{\circ} 0-07^{1} 0$ & $\Sigma_{g}^{+}-\Pi u$ & .240 & 16.2 & 0.057 \\
\hline 8) & $10^{\circ} 0-01^{1} 0$ & $\Sigma_{g}^{+}-\Pi_{u}$ & .225 & 13.9 & 0.044 \\
\hline 9) & $00^{\circ} 2-10^{\circ} 1$ & $\Sigma_{g}^{+}-\Sigma_{u}^{+}$ & & 10.4 & \\
\hline 10) & $01^{1} 1-11^{1} 0$ & $\Pi_{g}-\Pi_{u}$ & & 10.8 & \\
\hline
\end{tabular}


and 2) and also 5) and 6) for $v=2$. Transitions 1) and 5) also show that the $v_{3}$ moment is three times larger than the pure $v_{2}$. The mixed transitions are smaller yet.

The energies of these transitions are determined by the change in $E_{v i b}+E_{\text {rot }}$. An infrared spectrograph of $\mathrm{CO}_{2}$ emission appears as a band of lines with $\lambda_{0}$ the band center wavelength. The nearly evenly spaced lines on the long wavelength side comprise the $P$ branch, $\Delta \mathrm{J}=+1$. Those on the short wavelength side are in the R branch, $\Delta J=-1$. The rotational contributions to the emission energy are

$2 B(J+1)$ for $R$ branch

$$
\begin{aligned}
E_{J}-E_{J}= & -2 B J \text { for } P \text { branch }
\end{aligned}
$$

The spacing between the lines is $2 \mathrm{~B}\left(=0.8 \mathrm{~cm}^{-1}\right)$ when a) lines are present. When $Q$ transitions are allowed they fall right at band center with a slight spreading caused by small differences in $B(E q$. II-2) for the two transition levels.

There are three types of bands for $\mathrm{CO}_{2}$ (Herzberg, 45, p : 3801 .

1. $\Sigma-\Sigma$ transitions for which $l=0$ in both the upper and lower states. Only $\Delta J= \pm 1$ can occur so there is no Q branch. 


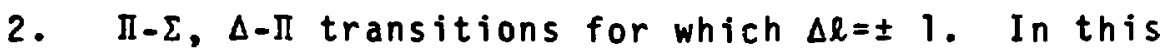
case $\Delta J=0, \pm 1$, so a $Q$ branch appears which has stronger transitions than the $P$ or $R$. For transitions 5) through 8) in Table II-2, the Q-transition $A$ values are about twice the $P$ and $R$ average (Statz et al,,66) but have not been shown in that table.

3. $\Pi-\pi, \Delta-\Delta$ transitions for which $\Delta l=0$, but $\ell \neq 0$. These bands also have $P, Q$ and $R$ branches $(\Delta J=0, \pm 1)$, but in this case the $Q$ branch is weak.

These bands describe the characteristics of gaseous absorption and emission and also laser transitions. The laser transitions relevant to the present work are shown in Fig. II-l as the 9- and 10-um normal transitions, the $11-\mu m$ hot-band $(011+110)$ transition, and the $10-\mu m$ sequence-band $(002+101)$ transition. A laser equipped with a grating in the cavity can lase on many $P$ and $R$ lines of these transitions. The individual lines are designated by $P(20)_{10}$ with 20 the $J$ of the lower state and 10 the band indicator. Because of the symmetries discussed above, the normal bands have only even lines while. the sequence band has only odd, thus there can be no confusion, between the two, in the use of the above designation. The 11-um band has both even and odd lines. 


\section{B. Energy Transfer Cross Sections}

Energy transfer collisions between molecules may be defined as those in which the internal energy states (vibration and rotation) after the encounter are different from those before. Types of energy transfer processes are traditionally designated by two of the three letters $V, R$, and $T$ to indicate vibration, rotation, and translation.

The choice of the two signifies which degree of freedom is losing energy and which is gaining. This notation is somewhat ambiguous, as collisions will in general involve changes in all three. In practice the two really indicate which degrees are of the most interest for the particular application at hand. With this in mind, then, this study has addressed $V-V$ and $R-R$ collisions in $\mathrm{CO}_{2}$.

For precise discussions a notation is required in which the specific state-to-state reaction is described. Toward this end, I will signify molecules by

$$
\left(v_{1} v_{2}^{l} v_{3}, j m\right)_{A}
$$

This specifies the vibrational and rotational quantum numbers of the molecule. The A signifies molecule $A$ as in $A+B \rightarrow A^{\prime}+B^{\prime}$ reaction notation. Any letters can be dropped for brevity if no ambiguity results. A general $\mathrm{CO}_{2}$ energy transfer collision is tien designated

$$
\begin{aligned}
\left(v_{1} v_{2}^{l} v_{3}, j\right)_{A} & +\left(v_{1} v_{2}^{L} v_{3}, J\right)_{B} \leftrightarrow\left(v_{j} v_{2}^{l^{\prime}} v_{3}^{\prime}, j^{\prime}\right)_{A} \\
& +\left(v_{j} v_{2}^{\prime} L^{\prime} v_{3}, J\right)_{B}+\Delta E
\end{aligned}
$$


The $\Delta E$ is the kinetic energy required to conserve the total energy and is the negative of $\Delta E_{V I B}+\Delta E_{R O T}$. The $A, B$ are useful for discussing the effects of identical particles. The magnetic quantum numbers are summed over in calculations so are dropped.

An energy transfer collision is a quantum mechanical event whose final state probability depends on the initial state and the interaction potential. For atoms and molecules this generally means there will be a shortrange, strongly repulsive force and a long-range, weakly attractive force. The short-range repulsion is due to electron distribution overlap. The attractive potential results from London dispersion forces (Van der Wals interaction) and static-electrical moments. $\mathrm{CO}_{2}$ has a static quadrupole moment but no static dipole moment.

Collision theory is difficult when nonspherical interactions are involved, when the state-changing matrix elements are large, and when both colliding particles have internal degrees of freedom. $\mathrm{CO}_{2}+\mathrm{CO}_{2} \mathrm{COllisions}$ require inclusion of all three effects. No theory exists at the present time which gives an adequate general description. However, there has been explosive growth in atom-molecule collision theory (Bernstein, 79) and some diatomic-diatomic work has appeared (Heil et al., 78). Can linear triatomics be far behind? 
The collision cross section, $\sigma$, is the fundamental result of experiments and theory. It represents the point at which calculations and lab results confront each other with hope of finding themselves in agreement or at odds. This is not always an easy decision because the state-tostate o's invariably require final-state sums and initial-state averages over some of the quantum numbers before a direct comparison to an experiment can be made. Often occupation probabilities of states are required in the sums (Pack, 80) which in turn means one must know the distribution function and the appropriate temperature. These requirements are sometimes difficult, especially in electrical discharge studies. It is hoped that the use of the uniform temperature hot cell in this work will make comparison of results a straightforward matter.

The theory of rotational relaxation is clearly in an Early stage of development (Lambert, 27, p. 115). The different calculational approaches described in that review do indicate, however, that the temperature dependence of the relaxation rates is an important parameter. Some a "roaches predict slightly positive temperature dependence for the relaxation while others predict a slightly negative dependence. The data compilation in the same work also shows interesting temperature dependencies. For hydrogen the energy 
transfer probability per collision increases with increasing temperature while for heavier molecules $\left(\mathrm{N}_{2}\right.$, $0_{2}, \mathrm{CO}$, and $\mathrm{CH}_{4}$ ) it decreases.

The rotation transfer investigated in this work involves 001 collisions of the type

$$
\begin{aligned}
(001, j)_{A} & +\left(v v_{2}^{\ell} v_{3}, J\right)_{B} \rightarrow\left(001, j^{\prime}\right)_{A} \quad I I-12 \\
& +\left(y_{i}^{\prime} v_{2}^{l} v^{\prime} v_{3}^{\prime}, J^{\prime}\right)_{B}+\Delta E
\end{aligned}
$$

Determining whether there are any restrictions on the quantum numbers involved is one goal of this experiment.

Some of the possible models for this reaction are discussed in the introduction and will be discussed in detail in Chapter III. The reservoir model imposes no constraint on $\sigma\left(j-j^{\prime}\right)$, while $\Delta j=2$ requires $j^{\prime}=j \pm 2$. In each of these the $B$-molecule state is unimportant. However, if the ground state $V-V$ process is important, the final 001 molecule is the B molecule. This reaction will be further discussed below as it is a resonant $V-V$ process.

In $V-V$ transfers, generally speaking, $\sigma$ temperature dependence is very important and $\Delta E$ is a key parameter. In the case of nonresonant collisions, $\Delta E>200 \mathrm{~cm}^{-1}$, SSH theory (Lambert, 77, p. 83) in many cases predicts the observed Landau-Teller positive temperature dependence of 
the collision probability, $P, 1 . e ., \operatorname{InP} \alpha T^{+1 / 3}$, so $\sigma$ is an increasing function of the temperature. The magnitude of the predictions, however, can show large errors. For the resonant case, $\Delta E<50 \mathrm{~cm}^{-1}$, SSH theory is capable of weakly positive or weakly negative temperature dependence. In general, $V-V$ theories can't reproduce the large body of experimental data that exists (Moore, 73 ). Certain resonant $V-V$ reactions, with $\Delta E \leq 25 \mathrm{~cm}^{-1}$, in which a dipole-allowed vibrational quantum is exchanged is an exception. In these circumtances simple theory gives very good results. Unadjusted calculations of $\sigma$ seem to agree with experiments in magnitude and $T^{-1}$ temperature dependence.

Mahan was the first to suggest that a long range potential could act between a vibrationally excited molecule and an unexcited one if the vibration was infrared active. He stated that the interaction potential would be the same as for two permanent dipoles except that the product of two vibrational transition dipole moments would be required instead of permanent moments (Mahan, 67). The dipole-dipole interaction (Jackson, 62, p. 102) is given by

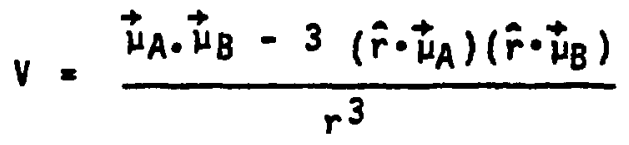


with the vector between the centers of molecules $A$ and $B$ with dipole moments $\overrightarrow{\vec{p}}_{A}$ and $\vec{\mu}_{B}$. The potential may be expressed in a form more convenient for calculations (Massey, 71, p. 1607) (Van Kranendonk, 63) as

$$
\begin{aligned}
& V=\frac{\mu_{A} \mu_{B}}{r^{3}} \sum-\frac{1}{3}(4 \pi)\left(\frac{6}{5}\right)(-1)^{m_{A}+m_{B}} C\left(1 ; 2 ; m_{A} m_{B}\right) \\
& x Y_{1 m_{A}}\left(\theta_{A}, \phi_{A}\right){ }^{r_{1 m_{B}}}\left(\theta_{B}, \phi_{B}\right) Y_{2},-m_{A}-m_{B}\left(\theta_{r}, \phi_{r}\right)
\end{aligned}
$$

The $C\left(J_{A} J_{B} J ; m_{A} m_{B}\right)$ is a $C l e b s c h-G o r d a n$ coefficient (Rose, 57) and $Y_{1 m}$ is a sphericai harmonic. The spherical angles represent the angles from $\vec{\mu}_{A}, \vec{\mu}_{B}$, and $\vec{r}$ to some arbitrary axis.

Pack's scattering amplitude (Pack, 80) is

$$
\left.\overrightarrow{f\left(K^{\prime}, \vec{k}\right)}=-(4 \pi)^{-1} 2 m_{R} /(h / 2 \pi)^{2}<\phi\left(\vec{K}^{\prime}\right) \psi_{A}{ }^{\prime} \Psi_{B} \cdot|V| \phi(\vec{K}) \psi_{A} \psi_{B}\right\rangle
$$

Here $\psi_{A}$ and $\psi_{B}$ are the internal wave functions. The $\phi, k$, and $m_{R}$ are the relative wave function, relative wave vector, and reduced mass, respectively. Pack calculates for the case of a general multipolemultipole interaction. He uses standard angular momentum and scattering theory techniques and a novel wave function cutoff radius, a, in his radial integral to obtain an expression for the differential cross section. 
For the case of transition dipoles this is

$$
\begin{aligned}
& \left.I\left(\vec{K}^{\prime}, \vec{K}\right)=\frac{K^{\prime}}{K}\left(2 m_{R} /(h / 2 \pi)^{2}\right)^{2} 2 \mu_{A}^{2} \mu_{B}^{2} c^{2}\left(j_{A}\right) j_{A^{\prime}} ; 00\right) \\
& \quad \times c^{2}\left(j_{B} 1 j_{B} ; 00\right) \frac{j \frac{j}{q^{2}}(q)}{}
\end{aligned}
$$

Here $j_{l}(q)$ is the spherical Bessel function and

$$
q^{2}=\left(k^{2}+k \cdot 2-2 K K^{\prime} \cos \theta\right) a^{2}
$$

is a dimensionless momentum exchange wave number. The angle $\theta$ is the center of mass scattering angle. The required integral over internal angles is performed in the standard way by letting $\vec{q}$ define the $z$ axis for spherical angles. The $\mu_{A}$ and $\mu_{B}$ represent the transition dipoles for the process under consideration.

The state-to-state cross section is obtained by integration over the scattering argle. Simpler procedures result if the integration variable is transformed from $\theta$ to $q$. The required dipole integral is then

$$
G=\int_{d}^{s} \frac{j_{T}^{2}(q)}{q} d q
$$

with $s=K^{\prime} a+K a$ and $d=\left|K^{\prime} a-K a\right|$. Pack shows this type of integral can be accomplished analytically and the 
dipole-dipole result is

$\left.\sigma=\frac{8 \pi}{(K a)^{2}} 2 m_{R} /(h / 2 \pi)^{2} \quad \mu_{A}^{2} \mu_{B}^{2} C^{2}\left(j_{A} 1 j_{A} ; 00\right) C^{2}\left(j_{B}\right) j_{B} ; 00\right) G \quad I I-i 6$ with*

$$
G=\frac{1}{4}\left[1-\frac{1}{q^{2}}+\frac{2 \sin q \cos q}{q^{3}}-\frac{\sin ^{2} q}{q^{4}}\right] \quad \mid s \quad \text { s } \quad \text { II }-17
$$

In all cases of interest $s \gg d$, so

$$
G=\frac{1}{4 d^{2}}\left[1-\frac{\sin (2 d)}{d}+\frac{\sin ^{2} d}{d^{2}}\right]
$$

This expression for $G$ goes to 0.25 at $d=0$, drops rapidly to zero for large $d$, and drops to 0.125 at $d \simeq 1.75$. This behavior is responsible for the resonant nature of the reactions. Pack shows that

$$
d=K a \frac{|\Delta E|}{2 E}
$$

when $E \gg \Delta E$.

$$
\text { At } 700^{\circ} \mathrm{K}, \mathrm{Ka}=117 \mathrm{~cm}^{-1} \text { while } E\left(\simeq .5 \mathrm{~m}_{R}\langle v\rangle^{2}\right)=641 \mathrm{~cm}^{-1} \text {, }
$$

so the cross section is down to $l / 2$ its $J E=0$ value when $\Delta E \simeq 19 \mathrm{~cm}^{-1}$. This strong dependence on $\Delta E$ only allows large cross sections when the rotational transitions are such that rotational and vibrational energy changes largely cancel to keep $\Delta E$ small. *Eq. (33) In Pack's paper, which should be equivalent to Eq. (II-17), has apparently been misprinted. The graph of $4 G_{f i}$ in his Fig. (2) agrees with EQ. (II-18) however. 
The Clebsch-Gordon coefficients in Eq. II-16 dictate the rotationally selective behavior of the reactions. They are (Rose, 57, p. 225)

$$
c^{2}\left(j_{A} 1 j^{\prime}{ }_{A} ; 00\right)=\frac{j_{A}+1}{2 j_{A}+1} \text { for } j_{A}{ }^{\prime}=j_{A}+1
$$

and

$$
c^{2}\left(j_{A} 1 j^{\prime} A ; 00\right)=\frac{j_{A}}{2 j_{A}+1} \text { for } j_{A}^{\prime}=j_{A}-1
$$

These are both $\simeq 1 / 2$ for large $j_{A}$. For $j_{A} \neq j_{A} \pm 1$ they are zero, thus each molecule may undergo a $P$ or an $R$ transition in this type of collision. Pack's wave functions do not allow for a bending mode so there can be no $\Delta J=0, Q$ transition. In real collisions one would think $Q$ transitions would be allowed if the collision involved molecules with bending vibrations.

Energy changes, as shown in Eq. II-11, in $P$ and $R$ transitions of a molecule are proportional to the initial $j$ and are approximately equal to $19 \mathrm{~cm}^{-1}$ at $j=23$ and 22 , respectively. It seems very likely that many combinations which sum to small $\Delta E$ would be possible for $\Delta E_{\text {vib }}<25$ $\mathrm{cm}^{-1}$.

In this experiment certain $v_{3}$-exchange $v-v$ reactions involving some highly excited vibrational states are examined. Each reaction is different in some aspect and requires a separate discussion. 
The collision process of foremost interest is

$$
\begin{aligned}
(001, j)_{A}+(001, J)_{B} & \leftrightarrow\left(002, j^{\prime}=j \pm 1\right)_{A}+\left(000, J^{\prime}=J \pm 1\right)_{B} \\
& \leftrightarrow\left(000, j^{\prime}=j \pm 1\right)_{A}+\left(002, J^{\prime}=j \pm 1\right)_{B}
\end{aligned}
$$

The 002 state lies at $4673 \mathrm{~cm}^{-1}$ (Rothman and Benedict, 78). In this case $\Delta E_{V}=-24 \mathrm{~cm}^{-1}$. The exchange process, shown on the lower line, is equivalent, so the cross section is doubled (Pack, 80). Also, one transition dipole is increased by $\sqrt{2}$ as shown in Table II-l and Eq. II-10. This should again double the cross section relative to collisions involving single $v_{3}$ quanta. This collision is considered important in laser energy storage but has not been examined experimentally.

The 002 level is the upper level in sequence-band lasing. The 101 ievel is the lower state of the $10 \mathrm{~mm}$ sequence band. This state also participates in a $v_{3}$ exchange process,

$$
\begin{aligned}
(001, j)_{A}+(100, j)_{B} & \leftrightarrow\left(000, j^{\prime}=j \pm 1\right)_{A}+\left(101, J^{\prime}=J \pm 1\right)_{B} \\
& \leftrightarrow\left(101, j^{\prime}=j \pm 1\right)_{A}+\left(000, J^{\prime}=J \pm 1\right)_{B}
\end{aligned}
$$

This process has $\Delta E_{v i b}=-22 \mathrm{~cm}^{-1}$, as 101 has an energy of $3715 \mathrm{~cm}^{-1}$. In the exchange process flower line) a bending mode quantum is seen to be transferred. A dipole-dipole matrix element with much weaker strength is 
involved and the process is usually neglected (Pack, 80 ). The direct cross section has been studied experimentally (Finzi and Moore, 75) at a single lower temperature. A measurement at $700^{\circ} \mathrm{K}$ should establish the temperature dependence and provide a further check of the Pack calculation.

The upper level of the hot band transition is the 011 level at $3004 \mathrm{~cm}^{-1}$. The $v_{3}$ transfer process

$$
\begin{aligned}
\left(00^{0} 1, j\right)_{A}+\left(01^{1} 0, j\right)_{B} & \leftrightarrow\left(00^{0} 0, j^{\prime}=j \pm 1\right)_{A}+\left(01^{1} 1, J^{\prime}=J, J \pm 1\right)_{B} \\
& \leftrightarrow\left(01^{1} 1, j^{\prime}=j, j \pm 1\right)_{A}+\left(00^{0} 0, J^{\prime}=J, J \pm 1\right)_{B}
\end{aligned}
$$

has $\Delta E_{v i b}=-12 \mathrm{~cm}^{-1}$ and also has a much weaker $v_{2}$ exchange reaction (lower line). This is a well established, resonant $V-V$ process (Burak et al., 73) (Doyennette et al., 75) although the agreement with theory (Pack, 80) is not good in the room temperature range. An interesting aspect of the cross section is that it would appear that molecule B could undergo a $Q$ transition $(\Delta J=0)$ with $\Delta l=0$ as shown in the upper line. As discussed in Section $A$ of this chapter, ho:rever, the $Q$ transition with $\Delta l=0$ is weak, so leaving it out is probably a good approximation.

The last $v_{3} v-v$ reaction to be examined is really only influential in rotational relaxation. This is the 
ground-state $V-V$ collision

$$
\begin{aligned}
(001, j)_{A}+(000, j)_{B} & \leftrightarrow\left(000, j^{\prime}=j^{\prime} \pm 1\right)_{A}+\left(001, j^{\prime}=j \pm 1\right)_{B} \\
& \leftrightarrow\left(001, j^{\prime}\right)_{A}+\left(000, j^{\prime}\right)_{B}
\end{aligned}
$$

The net number of molecules in the 000 and 001 vibrational states is seen to be unchanged. The exchange process is, curiously, a pure $R-R$ process so is not of interest as a $v_{3}$ transfer. In the direct process (upper line), the final rotation state of the 001 molecule is dependent on the initial $\mathrm{J}$ of the ground-state collision partner. This very resonant $\left(\Delta E_{v i b}=0\right)$ reaction must make some contribution to 001 rotational relaxation (Sharma and Kern, 71) (Moore, 73).

C. Rate Constants and Rate Equations

In gas experiments bulk properties are measured which are related to cross sections by event rates. For a sample in translational equiliorium, the rate at which a molecule $A$ undergoes a specified transition, with cross section $\sigma$, due to a collision with molecule $B$ is

$$
N_{B}<\sigma v>
$$

in which $N_{B}$ is the molecule $B$ density and the brackets 
indicate velocity distribution averaging. This is a pure rate so is independent of the reference frame, but centerof-mass quantities are normally used. Thus $\sigma$ is the center-of-mass cross section, and $v$ is the initial relative velocity. For averaging, then, the relative velocity distribution is required.

The rate constant is defined as

$$
k=\langle\sigma v\rangle
$$

The standard cgs unit is $\mathrm{cm}^{3} \mathrm{sec}^{-1}$ but for lab work torr ${ }^{-1} \sec ^{-1}$ is more convenient. Conversion requires multiplication of the latter by $k_{B} T / 1.333 \times 10^{3}$. A typical total collision rate at 1 torr is $10^{7} \mathrm{sec}^{-1}$. This provides an approximate upper limit that is useful in fast process studies. Determination of the rate constant for a process is usually the end point of experiments although a deconvolution is still involved in determining $\sigma$. A common approximation is $\langle\sigma v\rangle \approx\langle\sigma\rangle\langle v\rangle($ Pack, 80$)$.

The total event rate is obtained by multiplication of $N_{B} k$ by $N_{A}$. The observed total effect on molecules in a particular state depends strongly on the reverse reaction rate, $N_{B} N_{A}\left\langle\sigma^{\prime} v^{\prime}\right\rangle$. If the behavior of a level is to be modeled, a rate equation may be constructed by equating the rate of change of the level population to 
all the possible forward rates plus the associated reverse rates. This has the form

$$
\begin{aligned}
\dot{N}(\alpha, j)= & -N(\alpha, j)\left[N(\beta, J) k\left(\alpha j \beta j+\alpha^{\prime} j^{\prime} \beta^{\prime} J^{\prime}\right)\right. \\
& +\sum N\left(\alpha^{\prime}, j^{\prime}\right) N\left(B^{\prime}, J^{\prime}\right) k\left(\alpha^{\prime} j^{\prime} \beta^{\prime} J^{\prime}+\alpha j \beta J\right)
\end{aligned}
$$

In this equation $\alpha$ and $\beta$ represent the vibration quantum numbers and the sums are over all quantum numbers except $\alpha$ ano $j$. A complete description of a gas sample requires one such equation for each energy level. The result is a huge set of coupled nonlinear equations. Experiments are usually designed to reduce the number of levels of interest so that the equations can be managed.

Equilibrium for each reaction occurs when the densities involved reach values such that the forward and reverse rates balance. Thermal equilibrium, at some reservoir temperature $T$, occurs when all the levels reach their Boltzmann populations. At this point forward and reverse rates are equal in all reactions. For each reaction then,

$$
\begin{aligned}
N^{e}(\alpha, j) N^{e}(\beta, j) k\left(\alpha j \beta J+\alpha^{\prime} j^{\prime} B^{\prime} J^{\prime}\right) & =N^{e}\left(\alpha^{\prime}, j^{\prime}\right) N^{e}\left(B^{\prime}, J^{\prime}\right) \\
& \times k\left(\alpha^{\prime} j^{\prime} \beta^{\prime} J^{\prime}+\alpha j \beta J\right)
\end{aligned}
$$

This gives the so called microscopic reversibility 
relation between the forward and reverse rate constants

$$
k_{R}=k_{F} \frac{N^{e}(\alpha, j) N^{e}(\beta, j)}{N^{e}\left(\alpha^{\prime}, j^{\prime}\right) N^{e}\left(\beta^{\prime}, J^{\prime}\right)}
$$

The above rate equations determine the evolution of each leve? population. However, the changes in the densities are the real interest, so it is helpful to subtract the thermal equilibrium background. With $N(t)=N^{e}+n(t)$, the event rate becomes

$$
\left(N_{A}^{e}+n_{A}\right)\left(N_{B}^{e}+n_{B}\right) k_{A B}
$$

In most situations $N_{B} \simeq N_{B}^{e} \gg n_{B}$, i.e., the collision partner density is large and virtually unaffected by the process of interest. With this approximation and with the equilibrium terms subtracted, each equation takes the form

$$
\begin{aligned}
& \dot{n}(\alpha, j)=-n(\alpha, j) \sum N(\beta, J) k_{F} \\
& +\sum n\left(\alpha^{\prime}, j^{\prime}\right) N\left(\beta^{\prime}, J^{\prime}\right) k_{R}
\end{aligned}
$$

These steps cause linearization of the equations and allow simpler initial conditions since $n(t=0)=0$ for all but the initially perturbed state.

The discussion above implies some interesting characteristics for rate equation systems. For one thing equations like Eq. II-26 can be treated by linear vector space techniques. Matrix analysis, as an eigenvalue 
problem (Steinfeld, 78, p. 19), shows the general form of the solution is

$$
A+\sum_{i} B_{i} e^{-\gamma_{i} t}
$$

This is not an analytic solution unless one knows the eigenvalues, $Y_{j}$, and the associated eigenvectors, however, it is useful in that the explicit time dependence is exhibited.

Another important characteristic is that equilibrium for a reaction is a result of the forward and reverse characteristics of the collision, while $k$ determines the transient behavior in returning to equilibrium after a perturbation. If the levels involved in a reaction are essentially in equilibrium with each other, $k$ is no longer having an observable effect. Thus, if a specific $k$ is to be measured, its associated reaction must be knocked out of balance, i.e., the system must be perturbed on a time scale short compared to expected transient times. Also, this implies that different degrees of freedom can reach a quasi-equilibrium at different rates. For example, rotation exchange rates are large, so the transients are fast, and rotational relaxation occurs quickly. This means the ratios of the $J$ levels will have their equilibrium values although the whole vibrational leve? may still change in attaining vibrational equilibrium. 
This property can be used to advantage.

In $V-V$ studies it is normal to assume rotation is in equilibrium. This allows the sums over the rotational states to be performed for great simplification. In Pack's work (Pack, 80) the following sums are performed,

$$
k(T)=\sum_{j j} P_{j}(T) P_{j}(T) k(j J ; T) \quad I I-28
$$

with

$$
k(j J ; T)=\sum_{j \cdot J '} k\left(j J+j^{\prime} J^{\prime} ; T\right)
$$

This renders a pure $V-V$ rate constant. On the other hand, in rotational relaxation studies, vibration effects are usually assumed to be much slower and thus are neglected. Population conservation is also implied if the rate: equations are complete. This is of course a feature which holds regardless of equilibrium conditions. Since this requires the sums of the $i$ 's on the left of the equations to be zero, it must be that for every rate on the right side there is an equal but opposite rate elsewhere in the equations. This is indeed the case.

When numerically solving rate equations by computer, the concept of thermal equilibrium and population conservation provide aseful means of detecting errors. Once equilibrium populations have been calculated, the subprogram for the h's may be tested. The returned derivatives should be zero for equilibrium densities. 
Also total population can be computed and compared at initial and final times to assure oneself there is no significant error buildup in the many numerical steps leading to final solutions.

The rate constant is independent of pressure, but pressure still plays an important role in experiments. The transients observed depend on effective time constants,

$$
\tau=\left(N_{B} k_{A B}\right)^{-1}
$$

For kinetic processes, then, the time constants are inversely proportional to the pressure. In the lab it is wise to check that data have the appropriate linear pressure dependence. This is some assurance that the observations are being interpreted correctly.

In most rate constant experiments results of $\tau^{-1}\left(\sec ^{-1}\right)$ are plotted vs pressure (torr). Straight line fits to such data give $k\left(t_{0 r r}^{-1} \mathrm{sec}^{-1}\right)$ as the slope. The quality of the fit is also a good indication of experimental errors. The fitting errors are usually used to determine the quoted standard deviation. A systematic deviation from linearity in the data may also imply systematic experimental errors such as inadequate frequency response of the detection system.

A dependence of the $\tau$ 's on pumping intensity can be another indication that something is wry. During the 
pump pulse, coherent and nonlinear processes can affect the probe signal if the pump is very intense. After the pump pulse, there should be no intensity dependence as long as $N_{B}$ is not changing.

D. Double Resonance Spectroscopy

Any experiment which employs two sources of resonant electromagnetic waves in the sample can be called double resonance spectroscopy (DRS). The technique originated in the microwave spectroscopy of spin and rotation transitions in the $1950^{\circ} \mathrm{s}$. Its use in the infrared did not develop until lasers were invented making powerful monochromatic pump sources available.

In each stage of this experiment two $\mathrm{CO}_{2}$ lasers with different frequencies copropagate through a heated $\mathrm{CO}_{2}$ absorption cell 2.5 meters in length. Both frequencies undergo resonant absorption and the beams overlap as well as possible so the same group of molecules is sampled by each. One laser beam is very intense and has a short duration compared to relaxation times of interest. The other beam has constant intensity and is very weak. The former is called the pump; the latter is the probe. The probe has a negligible effect on the gas, but may undergo considerable attenuation in passing threugh the cell. The pump, on the other hand, must be intense enough to produce 
a substantial population transfer to the upper level associated with its aborption.

Thus, after the pump pulse, an excess of molecules in the upper energy state exists along the beam path. In returning to equilibrium the sample is expected to undergo, first rotational relaxation, then vibrational relaxation, and eventualiy give the excess internal energy up to translation. The probe beam acts as a monitor of the influence this process has on its two resonant ieveli. Population changes become transients on the derected transmission of the beam. In many cases the lower probe level is affected on a very slow time scale, so the signal reflects only the response of the upper level.

There are two general cases of pump and probe arrangems=nts; these are three-leve? and four-level colpling. In three-level measurements the pump and probe have a commmon upper level. In this case the probe directly measures the decay of the pumped level in its return to equilibrium.

The four-level arrangement employs no common level. If a sizeable energy transfer cross section exists for exchange of energy between the pumped level and the probe level, the probe transmission will reflect this transfer. The three-level signals depend strongly on the total 
rate out of the pumped level and are useful in rotational decay, or $\tau_{\text {rot }}$ measurements. The four-level signals depend on specific energy transfers and are useful for $J-d e p e n d e n t$ rotational relaxation and $V-V$ relaxation.

In this experiment many pump and probe combinations (designated by pump/probe) have been utilized in the study of 001 energy transfer. The three-level combinations $P(10)_{g} / P(10)_{10}, P(22)_{g} / P(22)_{g}, P(34)_{g} / P(34)_{10}$, and $P(22)_{10} / P(22)_{9}$ have been used to study the behavior of the rotational $\tau$ rot for the level 001 . A crude three-level measurement of $\tau_{\text {rot }}$ for 100 was also accomplished via $P(22) 10^{/ R(22)} 10^{\circ}$ Similarly, $\tau_{\text {rot }}$ for 020 was measured with the analogous 9-um lines. The response of neighboring 001 rotation levels was observed using the combinations $P(24)_{9} / P(24+\Delta J)_{10}$ with $\Delta J= \pm 2$, $\pm 4, \pm 6, \pm 10, \pm 14,-18$.

Vibration transfer of $v_{3}$ quanta from 001 to 002 , 101, and 011 was monitored by $P(24)_{g} / P(23), 10$ and $P(24)_{9} / P(24){ }_{1}$ (a) so $\left.P(24){ }_{1} / P(24), 1\right)$. These may be recognized as sequence-band $(002+101)$ and hot-band $(011+110)$ probe lines. The transitions used are schematically indicated in Fig. II-l.

The strength of the pump beam and the absorption of the probe do not explicitly affect the rate constants in DRS. However, they do determine whether a sufficient 
signal-to-noise ratio will result, so estimates are needed in experimental design. Also modeling and fitting programs require estimates of pump and probe properties. Thus some discussion of the formulas involved is required.

The absorption a laser beam experiences in traversing a length $\&$ of sample is determined by $e^{-a l}$, with the absorption coefficient

$$
\alpha=-\left(N_{l}-N_{u}\right) \sigma
$$

The absorption cross section, $\sigma$, depends on the transition lineshape in the absorbing gas. The $\mathrm{CO}_{2}$ laser produces a very narrow line at the line center absorption frequency of the gas. In this case $\sigma$ can be expressed in terms of the FWHM line width, $\Delta \nu(H z)$, as

$$
\sigma=\frac{\lambda^{2} A}{8 \pi \Delta \nu}
$$

Inhomogeneous Doppler broadening has a Gaussian lineshape while homogeneous pressure broadening has a Lorentzian shape. The Doppler width is given by

$$
\Delta v_{D}=(\langle v\rangle / \lambda)(.5 \pi \ln 2)^{\frac{1}{2}}
$$

with

$$
\langle v\rangle=\left(8 k_{B} T / \pi m\right)
$$

Here $m$ is the $\mathrm{CO}_{2}$ mass. At $700^{\circ} \mathrm{K}$ for $\mathrm{P}(20)_{10}$ $(\lambda=10.6 \mu \mathrm{m})$, this is about $57 \mathrm{MHz}$. The full pressure 
width is given by

$$
\Delta v_{p}=\left(\pi T_{2}\right)^{-1}
$$

in which $T_{2}$ is the collisional relaxation time of the polarization and is inversely proportional to the pressure. For $\mathrm{CO}_{2}, \mathrm{~T}_{2}^{-1}=2 \times 10^{7} \mathrm{sec}^{-1}$ torr ${ }^{-1}$. Thus

$$
\Delta v_{p}=6.7 \mathrm{MHz} / \mathrm{torr}
$$

In the present work operating pressures of 1 to 20 torr are needed so the two broadening mechanisms are comparable. The convolution of the two lineshapes is called the Voigt lineshape. The width can be approximated a s

$$
\Delta v^{2}=\Delta v_{D}^{2}+\Delta v_{p}^{2}
$$

The linecenter absorption coefficient is linear with pressure in the Doppler regime and independent of pressure when $\Delta v_{p}$ dominates, a condition which sets in above 50 torr.

At 1 torr and $700^{\circ} \mathrm{K}$, for example, $N_{\ell}-N_{u}$ is about $2 \times$ $10^{13}$ for $P(20)_{10}$, so the above formulas yield

$$
\begin{aligned}
& \sigma=2 \times 10^{-15} \mathrm{~cm}^{2} \\
& \alpha=0.4 \% / \mathrm{cm} \\
& \alpha \ell=1.0
\end{aligned}
$$


Any change in $N_{u}$ or $N_{\ell}$ may be detected as a change in the detected absorption cell transmission. If $\mathrm{N}_{\mathrm{u}}$ increases, highar transmission results; if $\mathrm{N}_{\ell}$ increases, the transmission is reduced. The observation of such changes is the task at hand.

If a high power laser beam, such as the pump, has sufficient intensity, each molecule will oscillate between the upper state and the lower state. For the whole sample then, half the molecules will be in one state and half in the other. This is a state of complete saturation of the absorption. An increase in intensity of the pump will then be transmitted with no further absorption. The characteristic intensity, at which saturation begins to set in, is called the saturation intensity, Is. For a Doppler broadened medium the expression for Is (Yariv, 75, p. 170) is

$$
I_{s}=\frac{\pi h \omega \Delta \nu}{\tau_{c} A \lambda^{2}}
$$

At one torr and $700^{\circ} \mathrm{K}$ with $\lambda=10.6 \mu \mathrm{m}, \tau_{c}=10^{-7}$ sec, $\Delta v=57 \mathrm{MHz}$, one finds $I_{s}=672 \mathrm{~W} / \mathrm{cm}^{2}$. This value indicates that for $1-\mathrm{cm}$ beams a probe of several watts will produce no undesirable effects on the populations while a pump of a megawatt or so will really make things happen. These are typical values for $\mathrm{CO}_{2}$ pump and probe 1 asers. 
For modeling the pump in rate equations an expression for the pumping rate is needed. The rate per molecule is given (Yariv, 75, p. 165) by

$$
W\left(\sec ^{-1}\right)=\frac{\lambda^{2} I A}{4 h \omega \Delta \nu}
$$

The pumping rate between $N_{\ell}$ and $N_{U}$ is then given by

$$
\left(N_{\ell}-N_{u}\right) W
$$

The sign of the population-difference coefficient will dictate whether absorption or gain will occur. As the pumping proceeds $N_{u}$ approaches $N_{\ell}$ in value and the transition rate saturates as it should.

In absorption experiments it is usually desirable to have the al large. The sample is then termed an optically thick medium and the analysis must take this into account.

An expression for the probe signal voltage in terms of population change can be derived by considering the transmission through the hot cell as depicted in Fig. II-3. The probe detector sigrial vs time is shown for three cases of interest.

In practice the probe is, indeed, turned on and off as indicated. However, the duration of the pulse is long compared to experimental times, so the probe is continuous for all practical purposes. 

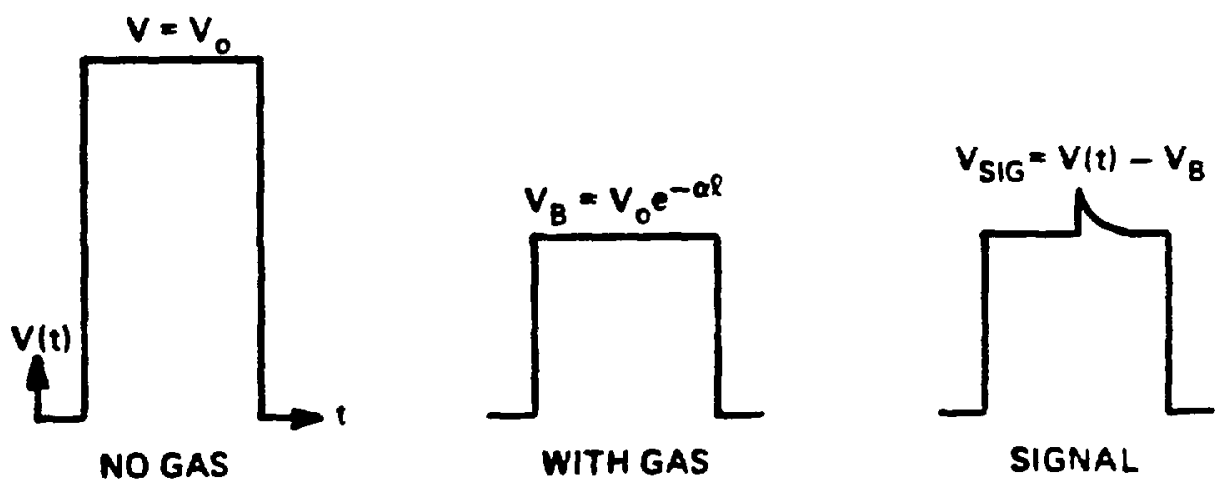

Figure II-3. Probe Transmission vs. Time

The probe intensity transmitted through the cell is shown for three conditions of interest: 1) no gas, 2) with gas, and 3 ) with a double resonance signal superimposed. 
With no gas in the cell there is complete transmission and $V=V_{0}$. With gas in the cell the probe experiences exponential absorption. The transmission signal is then $V=V_{0} \exp (-\alpha l)$ with $\alpha=\left(N_{l}^{e}-N_{u}^{e}\right) \sigma$ since equilibrium conditions prevail. This absorption will determine the baseline voltage, $V_{B}$, seen on an escilloscope trace.

The signal of interest is created when the pump produces a sudden disturbance in the upper level,

$$
N_{u}(t)=N_{u}^{e}+n(t)
$$

With the probe monitoring this level, the detection system will register the difference as the signal,

$$
v_{s}=v(t)-v_{B}
$$

If $N_{\ell}^{e}$ is unaffected

$$
\begin{aligned}
v_{s} & =v_{0} e^{-\left(N_{l}^{e}-N_{u}(t)\right) \sigma l}-v_{0} e^{-\alpha l} \\
& =v_{0} e^{-\left(N_{l}^{e}-N_{u}^{e}-n\right) \sigma l}-v_{0} e^{-\alpha l} \\
& =v_{0} e^{-\alpha l}\left(e^{n \sigma l}-1\right) \\
& =v_{B}\left(e^{n \sigma l}-1\right)
\end{aligned}
$$

This can be inverted to give an expression for population change,

$$
n=\frac{1}{\sigma l} \ln \left(1+\frac{V_{S}}{V_{B}}\right)
$$


In most cases $V_{S} \ll V_{B}$ so

$$
n=\frac{V_{s}}{\sigma \ell V_{B}}
$$

The linearity of the rate equations is a sufficient condition for the above arguments to hold. If the subset of rate equations being dealt with is not linear, the arguments may not be true. On the other hand, the linearity may not be a necessary condition. As long as the lower energy level populations are unaffected, to a good approximation, by the pumping, it seems reasonable that the time dependence of the relaxation would not depend on $z$ (the probe beam coordinate through the cell). 


\section{CHAPTER III}

\section{RELAXATION MODEL STUDIES}

Extensive use of computer simulation was not intended initially; simple beginnings evolved into heavier and heavier usage. Numerical modeling was such an aid to understanding the relaxation phenomena that it became an indispensible tool. What was first regarded as an unnecessary complication was soon viewed as a powerful technique for gaining insight into the behavior of the complex processes.

Four collision models of $R-R$ transfer were studied. The results have indicated how observations could be interpreted in terms of large and small $\Delta \mathrm{J}$ transfer and which characteristics of the signals should be most carefully measured. A multilevel model for $V-V$ transfer has also proven to be useful. The intuition gained from it, ultimately, resulted in a simple but accurate analytic model for the reactions of interest.

These model studies are discussed in the following sections of this chapter. All in all the computer modeling required has been a very satisfying aspect of the investigation. 
A. Rotation Transfer Models

The rotation transfer reaction is

$$
(001, j)_{A}+(B, j)_{B} \rightarrow\left(001, j^{\prime}\right)_{A}+\left(B, J^{\prime}\right)_{B} \quad \text { III-J }
$$

With $n(j)$ representing the change in $(001, j)$, the rate equation is

$$
\begin{aligned}
& \dot{n}(j)=-n(j) \sum N_{G}(J) k\left(j \jmath+j^{\prime} J^{\prime}\right) \\
& +\sum n\left(j^{\prime}\right) N_{G}(J \cdot) k\left(j^{\prime} J^{\prime}+j J\right)
\end{aligned}
$$

Here $N_{G}$ is the total gas density and the sums are over $j^{\prime}, J$, and $J '$. Also from Eq. II -25 ,

$$
\begin{aligned}
k\left(j^{\prime} J^{\prime}+j J\right)= & k\left(j J+j^{\prime} J^{\prime}\right) \frac{g(j) g(J)}{g\left(j^{\prime}\right) g\left(J^{\prime}\right)} \\
& x \exp \left[\varepsilon\left(j^{\prime}\right)+\varepsilon\left(J^{\prime}\right)-\varepsilon(j)-\varepsilon(J)\right]
\end{aligned}
$$

with

$$
\varepsilon(J)=B J(J+1) / k_{B} T
$$

The approach employed for numerical modeling of this system has been previously published (Preston and Pack, 78). Their procedure incorporates both of the vibration levels associated with the pump source. The $J$ levels for each vibration are included as are pumping rate terms which couple the two vibrations through the appropriate two rotation states. The rest of the gas provides a reservoir of collision partners. The rate constants are supplied in the form of a k-matrix, the elements of which 
may be read in or calculated. The reverse elements are calculated by Eq. III-3, so microscopic reversibility is assured. A copy of the original code was available* and revisions of it have been used in the studies to be described. The program utilizes a predictor-corrector subprogram with error checking to solve the rate equations. All runs were on the CDC 7600 with execution times of about 0.3 seconds.

Some general changes in the code were required. An accurate value of the $700^{\circ} \mathrm{K}$ partition function, when compared to Eq. II-5, demanded an increase in the number of $\mathrm{J}$ levels from 26 to 35 . Additional coding, to check thermal equiliorium and population conservation, was also added.

A total of four collision models were investigated. These are the reservoir model, the $\Delta J=2$ model, the 000 $V-V$ model, and the $\mathrm{He}+\mathrm{CO}_{2}$ model. The reservoir model equations can be solved analytically. This provides insight into the relaxation process and also serves as a convenient check on the code. This model will be discussed first.

*The code was made avallable by R. T. Pack and J. C. Goldstein of Los Alamos National Laboratory. 
1. The Reservoir Model

If the assumption of no $J$ selectivity is made for rotation transfer, the reservoir model results. It is widely used in laser simulations (Smith and Thompson, 78, p. 119) because of the simplification it allows while still giving an adequate representation of the relaxation for most applications.

The results follow if the basic assumption is made that $k\left(j J+j^{\prime} J^{\prime}\right)$ is proportional to the final state Boltzmann factor; that is,

$$
k\left(j J+j^{\prime} J^{\prime}\right)=k_{0} g\left(j^{\prime}\right) g\left(J^{\prime}\right) \exp \left[-\varepsilon\left(j^{\prime}\right)-\varepsilon\left(J^{\prime}\right)\right] \quad \text { III }-4
$$

with $k_{0}$ independent of rotation. The reverse constant is thus

$$
k\left(j^{\prime} J^{\prime}+j^{\prime}\right)=k_{0} g(j) g(J) \exp [-\varepsilon(j)-\varepsilon(J)] \quad \text { III-5 }
$$

The proper equilibrium behavior is assured as the ratio $k_{R} / k_{F}$ is seen to satisfy the reversibility condition, Eq. III 3 .

When these expressions are substituted into Eq. III-2, the sums become decoupled, and

$$
\begin{aligned}
& \dot{n}(j)=-n(j) k_{0} \sum_{j} N_{G}(J) \sum_{j} g\left(J^{\prime}\right) e^{-\varepsilon\left(J^{\prime}\right)} \sum_{j^{\prime}} g\left(j^{\prime}\right) e^{-\varepsilon\left(j^{\prime}\right)} \\
& \left.\left.+k_{0} g(j) e^{-\varepsilon(j)}\right\}_{j} g(j) e^{-\varepsilon(J)}\right\}_{V_{G}} N_{G}\left(J^{\prime}\right) \sum_{j,} n\left(j^{\prime}\right)
\end{aligned}
$$


The sums may each be identified as a total density or a rotational partition function (discussed in Section II.A.). Thus the rate equation rejuces to

$$
\dot{n}(j)=-n(j) N_{G} Q_{r}^{2} k_{0}+g(j) e^{-\varepsilon(j)} N_{G} Q_{r} k_{0} \sum_{j} n\left(j^{\prime}\right)
$$

The final sum on the right side is the total change in the vibrational level,

$$
n_{v}=\sum_{j,} n\left(j^{\prime}\right)
$$

When rotational equilibrium is reached the density change for a given level is

$$
n e(j)=\frac{n_{v}}{Q_{r}}(2 j+1) e^{-B j(j+1) / k_{B} T}
$$

With these relations the rate equation may be further simplified to

$$
\dot{n}(j)=-N_{G} Q_{r}^{2} k_{0}\left[n(j)-n^{e}(j)\right]
$$

The general solution of this equation is

$$
n(j)-n^{e}(j)=C \exp \left(-N_{G} Q_{r}^{2} k_{0} t\right)
$$

in which $C$ is the integration constant. In the present experiment an amount of population is quickly pumped to some level in 001 causing it to be out of equilibrium. As rotational relaxation proceeds, the pumped density will 
spread to all other 001 states until they are in equilibrium again. The total 001 population is increased to $N_{001}^{e}+n_{v}$. For fast pumping there are two boundary conditions. For the pumped level at $t=0, n(j)=n_{v}$, thus

$$
\left.n(j)=\left[n_{v}-n^{e}(j)\right)\right] \exp \left(-N_{G} Q_{r}^{2} k_{0} t\right)+n^{e}(j) \quad \text { III }-7
$$

For all the unpumped states at $t=0, n(j)=0$, so

$$
n(j)=n^{e}(j)\left[1-\exp \left(-N_{G} Q_{r}^{2} k_{0} t\right)\right]
$$

The quantity $N_{G} Q_{r}^{2} k_{0}$ is seen from $E q$. III-6 to be the total rate out of the pumped level. The pumped level decays with a time constant traditionally called $\tau_{r}$, the rotational relaxation time. For this case

$$
\tau_{r}=\left(N_{G} Q_{r}^{2} k_{0}\right)^{-1}
$$

For an effective rate constant of $N_{G} Q_{r}^{2} k_{0}=10^{7}$ torr $^{-1}$

$\sec ^{-1}$ and a gas pressure of 4 torr, for example, $\tau_{r}=25$ ns.

The rate coefficients from Eq. III-4 and Eq. III-5 may also be used to provide k-matrix elements so a numerical solution can be performed. The results of such an exercise are shown in Fig. III-2(a) for the pumped level and in Fig. III-3(a) for representative unpumped levels. The $k_{0}$ value is normalized so that $\tau_{r}=25 \mathrm{~ns}$. In the figure the exponential transients and final equilibrium behavior are evident. 
(a)
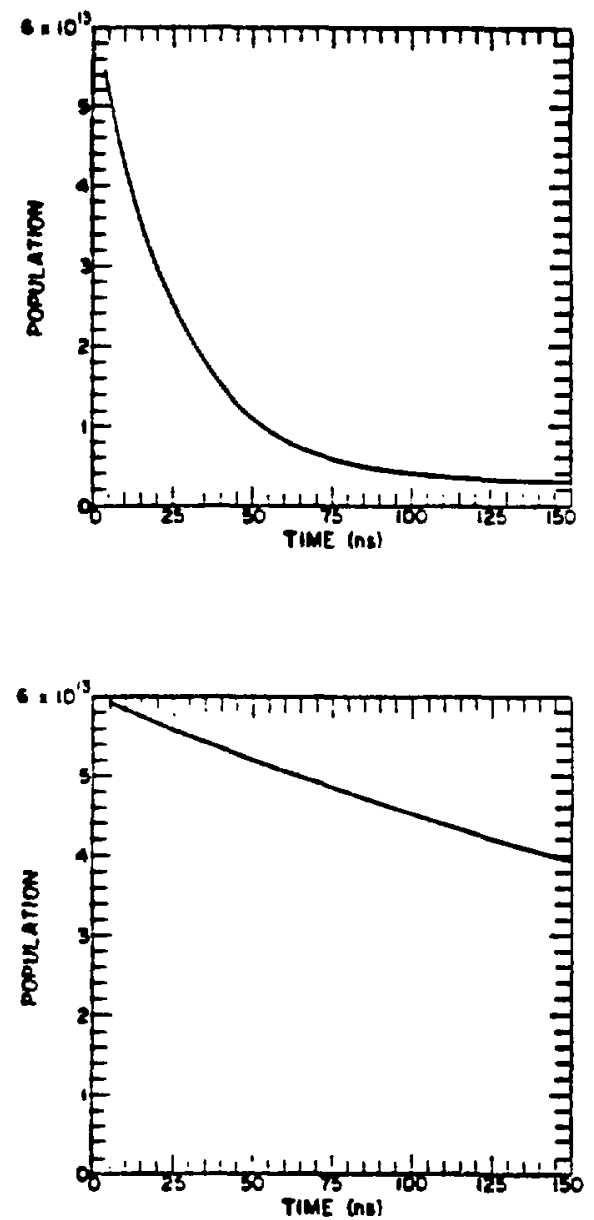

(c) (b)
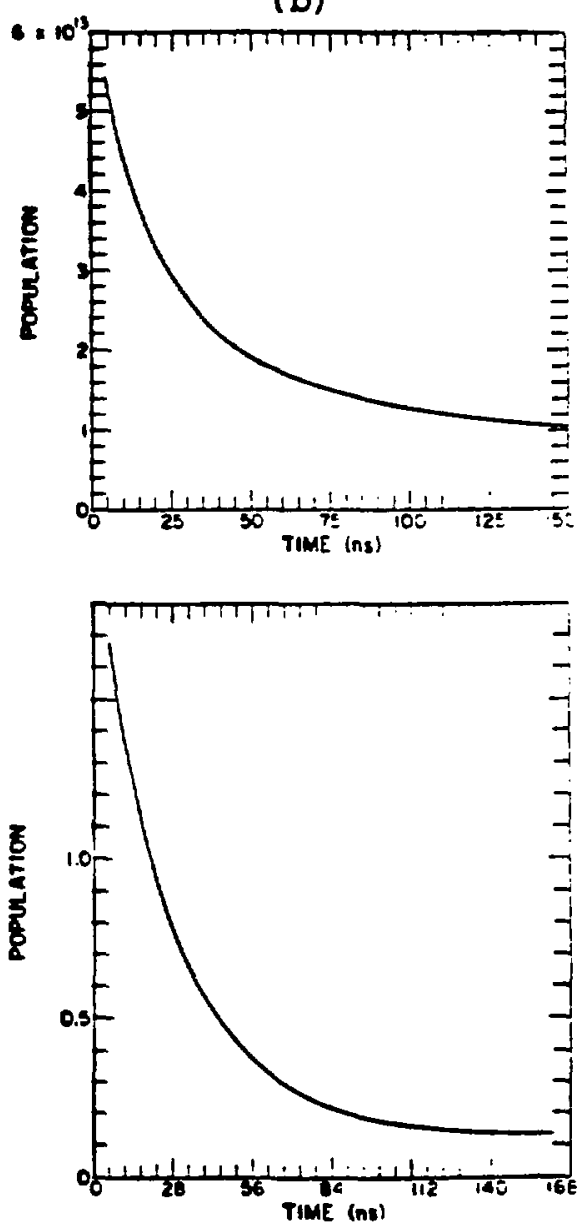

(d)

Figure III-2. The Pumped Level Response at 4 Torr

The pumped level transient is shown for four models:

(a) reservoir model, (b) $\Delta J=2$ model, (c) ground state $V-V$ model, and (d) $\mathrm{He}+\mathrm{CO}_{2}$ model. 
(a)

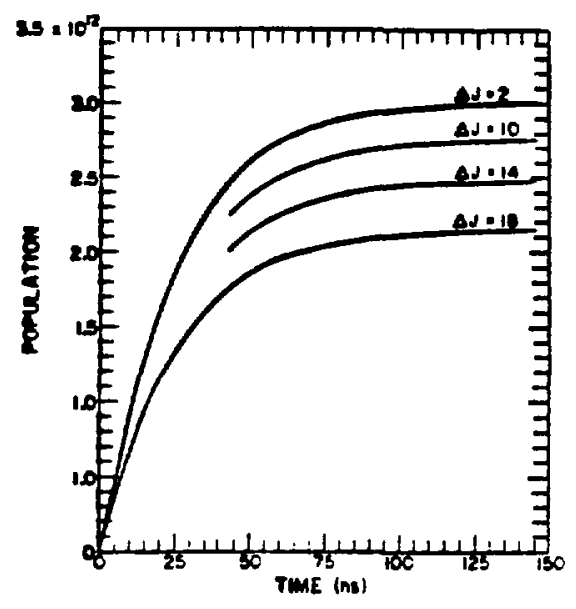

(b)

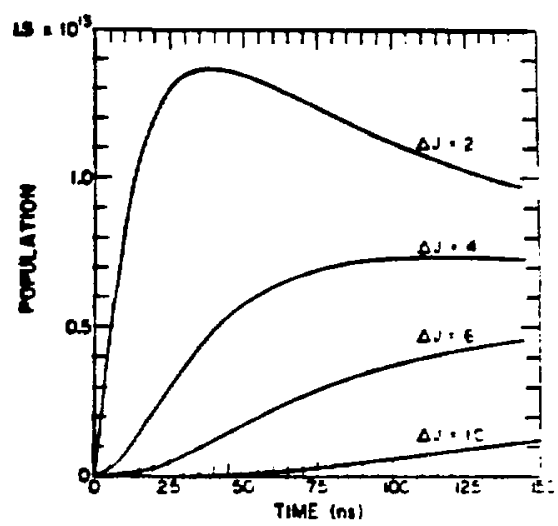

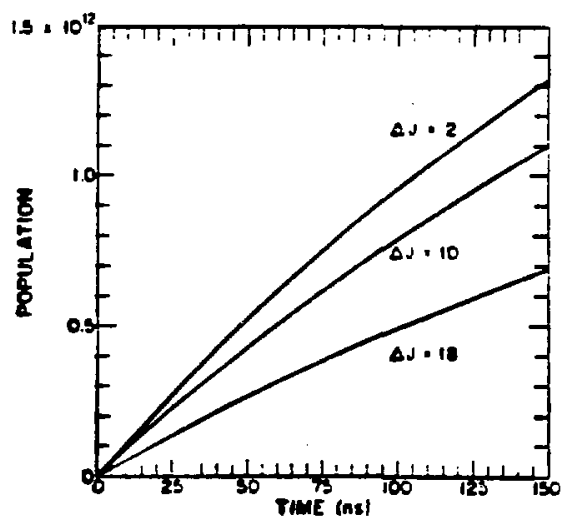

(c)

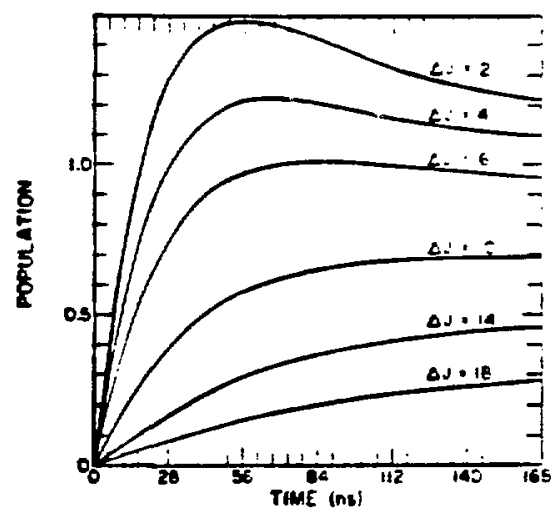

(d)

Figure III-3. The J-Dependence of the Relaxation at 4 Torr The response of neighboring rotational levels, with representative values of $\Delta J$, is shown for four models: (a) reservoir model, (b) $\Delta J=2$ model, (c) ground state $V-V$ model (note vertical scale), and (d) $\mathrm{He}+\mathrm{CO}_{2}$ model. 
2. The $\Delta \mathrm{J}=2$ Model

For this type of selectivity the reaction would be

$$
(001, j)+(B, J) \rightarrow\left(001, j^{\prime}=j \pm 2\right)+\left(B^{\prime}, J^{\prime}\right)
$$

Two previous investigations have used this model in their analysis (Carrol and Marcus, 68) (Jacobs et al., 74b).

The k-matrix for such a selection rule is completely determined if three assumptions are made:

$$
\begin{aligned}
& \text { 1. } k\left(j^{\prime}=j^{+2}\right)=k\left(j^{\prime}=j-2\right) \text {, } \\
& \text { 2. microscopic reversibility is obeyed, and } \\
& \text { 3. } \tau_{r}=\left[N_{G}\left(k_{+2}+k-2\right)\right]^{-1} \text { is fixed for the } \\
& \text { pumped level. }
\end{aligned}
$$

The procedure for construction of the matrix is illustrated in Fig. III-1 for a hypothetical system with 19 levels, in which the pumped level is $\mathrm{J}=9$. Each row represents an initial state, JJ, while the columns determine the final state, $J_{f}$. The diagonal elements play no role so are equated to zero. The two elements, $k$, shown for level 9, are determined by assumption 3 with $\tau_{r}=25 \mathrm{~ns}$. Then condition 2 fixes the diagonally opposite elements. At that point assumption 1 may be applied to get the second element in the new row. Iteration of conditions 1 and 2 fill the matrix to the upper left and to the lower right as indicated by the small arrows in the figure. The other off-diagonal elements are zero. 


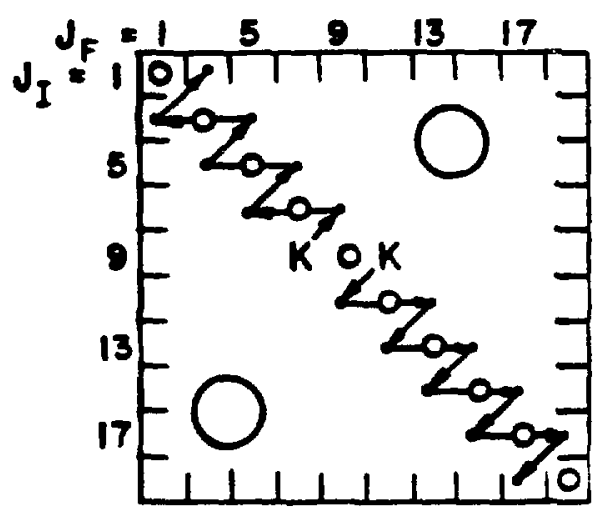

Figure III-I. The $\Delta J=2$ Rate Constant Matrix

The construction procedure for the $\Delta J=2$ rate matrix is indicated for a hypothetical system with $\mathrm{J}=1$ through $J=19$ for which $J=9$ is the pumped level. 
The results of the numerical solution with $\tau_{r}=25 \mathrm{~ns}$ and $J_{\text {pump }}=23$ are shown in Fig. III-2(b) for the pumped level. Fig. III-3(b) shows the neighboring levels. The very long risetimes for large $\Delta J$ and the oversnoot for small $\Delta J$ are characteristic features of the model.

3. The Ground State V-V Mode?

As discussed at the end of Section II-B, the reaction in this process is

$$
\begin{aligned}
\left(001, j_{A}\right)_{A}+\left(000, j_{B}\right)_{B} & \leftrightarrow\left(000, j_{A}^{\prime}=j_{A} \pm 1\right)_{A} \\
& +\left(001, j_{B}^{\prime}=j_{B} \pm 1\right)_{B}
\end{aligned}
$$

The important point is that this $V-V$ transfer is a rotational relaxation mechanism. No overall change in the 001 or 000 population is induced, but a change in the 001 rotational distribution does occur. The strength of this $v_{3}$ transfer cannot be estimated by comparison with Pack's final results as he sums over rotational distributions to get pure $V-V$ rate constants. The rotational, state-tostate $k$ values are needed to generate a $k$ matrix. These may be obtained from the cross-section formulas, Eq. II-16 and Eq. II -18 .

The notation for states becomes a little confusing at this point. The k-matrix elements are for transitions of the type $(001, j)+\left(001, j^{\prime}\right)$, for which $j=j_{A}$ and $j^{\prime}=j^{\prime}{ }_{B}$. 
A more convenient way to write the reaction is

$$
\begin{gathered}
\left(001, j_{A}+\left(000, j=j^{\prime} \pm 1\right)_{B} \leftrightarrow\left(000, j^{\prime}=j \pm 1\right)_{A}\right. \\
+\left(001, j^{\prime}\right)_{B}
\end{gathered}
$$

The rate equation for this process is

$$
\begin{aligned}
\dot{n}(j) & =-n(j) \sum N_{0}(J) k\left(j J+j^{\prime} j^{\prime}\right) \\
& +\sum n\left(j^{\prime}\right) N_{0}\left(J^{\prime}\right) k\left(j^{\prime} J^{\prime}+j^{j}\right)
\end{aligned}
$$

where $j$ and $j^{\prime} i m p l y 001$ states and $J$ and $J$ indicate 000 states with no reference to the $A$ and $B$ identifiers. Since $N_{0}(J)=N_{0} P(J)$, the desired forward coefficient may be expressed as

$$
k\left(j+j^{\prime}\right)=\sum_{j} P(J) \sum_{j,} k\left(j J+j^{\prime} J^{\prime}\right) \delta j, j^{\prime} \pm 1^{\delta} j,, j \pm 1 \quad \text { III }-13
$$

This is essentially an average over initial and sum over the final J states.

As was discussed in connection with Eq. II-19, the resonant behavior of such collisions is determined by the value of $d$ which in this case, with $\Delta E_{v}=0$, is

$$
d=K a \frac{\left|\Delta E_{r}\right|}{2 E}
$$

Large cross sections will result when one of the colliding pair increases its rotational energy while the other's decreases so significant cancellation occurs. This may happen in two ways, $\Delta E_{r}=\Delta E_{A}^{+}+\Delta E_{B}^{-}$or $\Delta E_{r}=\Delta E_{A}^{-}+\Delta E_{B}^{+}$. 
The superscripts indicate whether rotational energy increases or decreases. In the first case

$$
\begin{aligned}
\Delta E_{A}^{+} & =B j_{A} \cdot\left(j_{A}{ }^{+}+1\right)-B j_{A}\left(j_{A}+1\right) \\
& =B\left(j_{A}+1\right)\left(j_{A}+2\right)-B j_{A}\left(j_{A}+1\right) \\
& =2 B\left(j_{A}+1\right)
\end{aligned}
$$

and

$$
\Delta E_{B}^{-}=-2 B j_{B}
$$

In terms of $j+j$ ' notation, $j_{A}=j$ and $j_{B}=j '+l$ for this combination. Thus $\Delta E_{r}=-2 B \Delta j$ with $\Delta j=j^{\prime}-j$. For the other case, $\Delta E_{A}^{-}+\Delta E_{B}^{+}, j_{A}=j$ and $j_{B}=j^{\prime}-1$ so $\Delta E_{r}=+2 B \Delta j$. Both result in

$$
d=K a \frac{B|\Delta j|}{E}
$$

because of the absolute value.

When the colliding molecules, $A$ and $B$, have energy changes of the same sign, similar steps give $\left|\Delta E_{r}\right|=$ $2 B(j+j '+1)$. For $j$ 's of interest this value is out of resonance so can be neglected for some simplification.

The cross section is given by Eq. II-16 and Eq. II-18. As stated in relation to Eq. II-20 and Eq. II-2I, the Clebsch-Gordon coefficients are each $\simeq 1 / 2$ if $j_{A}$ and $j_{B}$ are not too smail. Therefore all the $j$ dependence in $\sigma$ is contained in $d$. 
The $k\left(j+j^{\prime}\right)$ equation can now be witten with the more restrictive delta functions,

$$
\delta_{J, j^{\prime}+1^{\prime} \delta^{\prime}, j+1}+\delta_{J, j^{\prime}-1^{\delta} J^{\prime}, j-1}
$$

Once the indicated sums are performed,

$$
k\left(j+j^{\prime}\right)=P\left(j^{\prime}+1\right) k\left(j, j^{\prime}+1+j^{\prime}, j+1\right)+P\left(j^{\prime}-1\right) k\left(j, j^{\prime}-1+j^{\prime}, j-1\right)
$$

The first $k$ on the right has $\Delta E_{r}=\Delta E_{A}^{+}+\Delta E_{B}^{-}$while for the second $\Delta E_{r}=\Delta E_{A}^{-}+\Delta E_{B}^{+}$. For these two cases, the associated $d$ values and crass sections are equal so

$$
k\left(j+j^{\prime}\right)=\langle\sigma\rangle\langle v\rangle\left[P\left(j^{\prime}+1\right)+P\left(j^{\prime}-1\right)\right]
$$

in which $k \simeq\langle\sigma\rangle\langle v\rangle$ has been substituted. An approximation for $\langle\sigma\rangle$ may be obtained by simply evaluating $\sigma(v)$ at $v=\langle v\rangle$. That is, with $E=1 / 2 m_{R}\langle v\rangle^{2}$ and $k=2 \pi m_{R}\langle v\rangle / h$. In addition, $a \simeq 4 \mathrm{~A}$ and $\mu=0.316 \times 10^{-18}$ esu may be used. The latter is from Pack's work (Pack, 80) while the former is the standard $\mathrm{CO}_{2}$ value.

With the resulting elements the desired $k$ matrix can be geneiated. Since $\sigma$ is only a function of $\Delta j$, only the $\sigma$ elements of the first row need be calculated. Column shifts can then be made to create the remainder of the upper right half of the array. Multiplication of the elements of a given column by $\langle y\rangle$ and the probabilities 
in Eq. III-15 completes this portion of the $k$ matrix. The lower left elements can then be generated by the reversibility condition.

Numerical solution results at 4 torr for this model are given in Fig. III-2(c) for the pumped level and in Fig. III-3(c) for neighboring levels. The relaxation is very slow relative to the other $R-R$ mechanisms. If the approximations are not too crude this reaction should produce a negligible effect. Calculation of $\tau_{\text {out }}$ by summing over the elements of the $j=23$ row gives

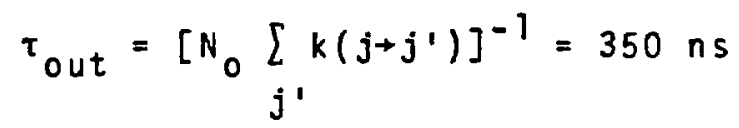

which is indeed long compared to $25 \mathrm{~ns}$.

4. The $\mathrm{He}+\mathrm{CO}_{2}$ Model*

The collision process described by this model is $(001, j)+\mathrm{He} \leftrightarrow\left(001, j^{\prime}\right)+\mathrm{He}$

This is a relatively simple reaction since the He has no internal degrees of freedom and no static moments. It is not obvious that this process has relevance to the present

*The k-matrix data was made available by R. T. Pack and J. C. Goldstein of Los Alamos National Lab. 
investigation. Its study has proven useful, however, in that it provided a starting point for coding the other $R-R$ studies, and the results do indicate the behavior of a realistic system.

The $k$ matrix in this instance was calculated for a gas temperature of $340^{\circ} \mathrm{K}$. The He pressure was 4 torr. Only 51 levels were required. Calculation of the total rate constant out of $j=23$ showed that $\tau_{\text {out }}=28 \mathrm{~ns}$. The closeness to 25 ns is coincidental but does allow easy comparison to the other models.

The numerical results are plotted in Fig. III-2(d) and Fig. III-3(d) for the pumped level and the $\Delta \mathrm{J}$ levels, respectively. The curve for the $J=23$ decay has the familiar exponential character. The unpumped level plots show a pronounced overshoot for small $\Delta J$ and also show the effect of the lower temperature on the final equilibrium densities.

5. Discussion of the $R-R$ Models

The first point is that the $000+001 V-V$ mechanism will probably be unimportant unless the approximations involved are really inappropriate. The three other systems show several important features.

First the pumped level decays are very similar regardless of the collision mechanism. Study of this type 
of data is thus expected to yield little information about mechanisms but is an easy way to get $\tau_{r}$. Information relevant to the energy transfer mechanism does seem to exist in the j-dependent relaxation. The reservoir model and the $\Delta J=2$ model represent two extreme cases. For the first, all risetimes are equal and the responses are monotonic. The second shows a pronounced overshoot with a very strong dependence of the risetime on $\Delta \mathrm{J}$. The selection rule apparently produces a cascade relaxation in which complete equilibration would take a very long time.

The realistic system, i.e., the $\mathrm{He}+\mathrm{CO}_{2}$ model, shows a somewhat intermediate behavior. There is overshoot for $\Delta J=2,4$, and 6 . The risetimes for larger $\Delta J$ are definitely on the increase,but the effect is not nearly as dramatic as in the $\Delta \mathrm{J}=2$ model. It seems that small $\Delta \mathrm{J}$ transfers are important but very large transfers are still possible.

The above studies of $R-R$ transfer indicate that the most important measurements to be made are $\tau_{r}$ by pumped level decay and the $r$ isetimes of the $\Delta J$ levels. The existence of overshoot, or its absence, is also important information. 


\section{B. Resonant $v_{3}$ Transfer Studies}

The primary objective of this experiment is the measurement of $k_{002}$. The intended method is to pump 001 with conventional $\mathrm{CO}_{2}$ laser lines while watching for population transfer to 002 with a sequence-band laser. From such data the value of $k_{002}$ should be obtainable by some type of fitting procedure. If $001+002$ were dominant in 001 relaxation, the measurement would be straightforward. But in order to use the $\mathrm{CO}_{2}$ laser as a pump source, the gas must be raised to a sufficient temperature to get some population into the lower pump level. This fact creates the principal difficulty with the method. Having many of the low lying vibrational levels populated means one cannot assume the ground state molecule is the only important collision partner. The increased number of important collision reactions creates a complicated relaxation system. Finding a convincing fitting model in such cases becomes a problem.

1. The Multilevel Computer Model

The pumping procedure described above causes the $\mathrm{CO}_{2}$ to divide into two systems which relax essentially independent of each other. The lower level, 020, is depleted and 001 becomes overpopulated. The extra 001 energy is dissipated mainly by populating higher lying states by $v_{3}$ exchange. The repopulation of the 020 
level proceeds via energy pickup from other low levels. These collisions are generally nonresonant and thus the relaxation is slower. If the pump pulse is kept short compared to $\tau_{r}$, the pumped population transfer will amount to approximately one $J$ level of the lower pump state, this is a few per cent of the total 020 density. The other low levels are thus expected to respond to the 020 deficit slowly with small net changes.

It would be expected that the migration of pumped energy from 001 would be dominated by reactions involving collisions with heavily populated low lying states. These would include 000 and 010 which account for $80 \%$ of the total density and possibly 020, $02^{2} 0$, and 100 which make up another 15\%. As discussed in II.B the process $001 \rightarrow 011$ is well understood as a $v_{3}$ transfer with $k_{011} \simeq 1.7 \times 10^{6}$. The $001+101$ transfer is also described in II.B. Rate constants for this reaction, and the closely related $001+021$ process with $\Delta E_{v}=-21 \mathrm{~cm}^{-1}$, have recently been reported (Finzi and Moore, 75). Those results are for a lower temperature, but calculations of $k$ vs $T(P a c k, 80)$, which agree with the experiment, predict $k_{101}=k_{021}=1.5 \times 10^{6}$ at $700^{\circ} \mathrm{K}$. Unfortunately, no measurements or predictions exist for $001+02^{2} 1\left(\Delta E_{v}=-25 \mathrm{~cm}^{-1}\right)$. However, the behavior of this transfer should be similar to $001+101$ and 021 . At first glance the reaction 
$00^{\circ} 1+02^{\circ} 0 \leftarrow 00^{\circ} 0+02^{2} 1$ might also seem important since $\Delta E_{v}=+25 \mathrm{~cm}^{-1}$, but it would require a $\Delta l=2$ transition which is dipole forbidden.

The above considerations imply that a rate model for 001 behavior could be devised providad the lower levels were also accounted for. The simplest way would be to assume they do not change at all. The more accurate alternative is to construct a rate system for them also. The lower level relaxation is important in $\mathrm{CO}_{2}$ laser action and has been extensively studied. The state of knowledge has been summarized previously (Judd, 75). Surprisingly, the processes are not well understood. A set of rate equations for the vibrational levels shown in Fig. II-i has been coded and incorporated into a series of programs which model the vibrational relaxation of the hot $\mathrm{CO}_{2}$ sample. The differential equations involved are solved by the Runge-Kutta method (4th order). To gauge numerical errors one must check that smaller step sizes give the same results. Population conservation and thermal equilibrium are also incorporated for debugging and error checking. The computer used in the $V-V$ studies was an Eclipse S-200 from Data General Corp.

The program had simple beginnings but was found so useful for interpreting preliminary data that frequent 
modifications were made to increase its capabilities. The levels included are those shown in Fig. II-l. Lower level relaxation is included. Also the levels $03^{7} 0,03^{3} 0$, and $11^{\prime} 0$ with about $5 \%$ of the density are included in the calculation of the partition function but are assumed constant in the solution, and none of their collisions are included. For the upper levels, only the aforementioned $v_{3}$ reactions are included. The rate constant for $001+02^{2} 1$ is assumed the same as $k_{101}$ and $k_{021}$. The value of $k_{002}$ used is $6 \times 10^{6}$, but it and the others were varied, as needed, for different studies.

Rotational equilibrium was assumed for the vibration states. The two rotation levels involved in the pumping were treated as two separate levels which relaxed via the reservoir model. These special levels were also allowed to undergo the same $V-V$ collisions as their parent populations 020 and 001 .

The program could estimate signal strength. This required absorption coefficients and detection parameters obtained from the lab.

The order of the preceeding paragraphs is a bit misleading as far as actual chronology is concerned. Understanding the vibrational relaxation, as described above, did not in fact occur until many comparisons of preliminary data with simulations were made. As the 
computer model evolved so did an intuition for which processes were important.

Early studies showed that the 011 level and the lower sequence-band levels played dominant roles in the 001 relaxation. At $700^{\circ} \mathrm{K}$ they are really more important than 002. The 011 level is so important it was deemed necessary to include, in the experiment, probe measurements at hot-band frequencies, so the model behavior of this state could be verified.

The system seemed so complex it was feared that the data from the sequence-band and hot-band signals would somehow have to be fitted directly from the computer model. As understanding developed, however, it became evident that 001 behavior was coupled to 011 and to the group $021,02^{2} 1$, and 101 while the $001+002$ process caused little drain on 007 . These insights allow a simple rate model, which can be solved analytically, for the various upper levels. The procedure is described in the following two sections.

2. The Three-Level Model

The output of the multilevel program for the levels relevant to this model is shown in Fig. III-4. The plots indicate the changes in the states $n_{001}$, n011. and $n_{F}$ after a saturating pump pulse. The curve labeled 


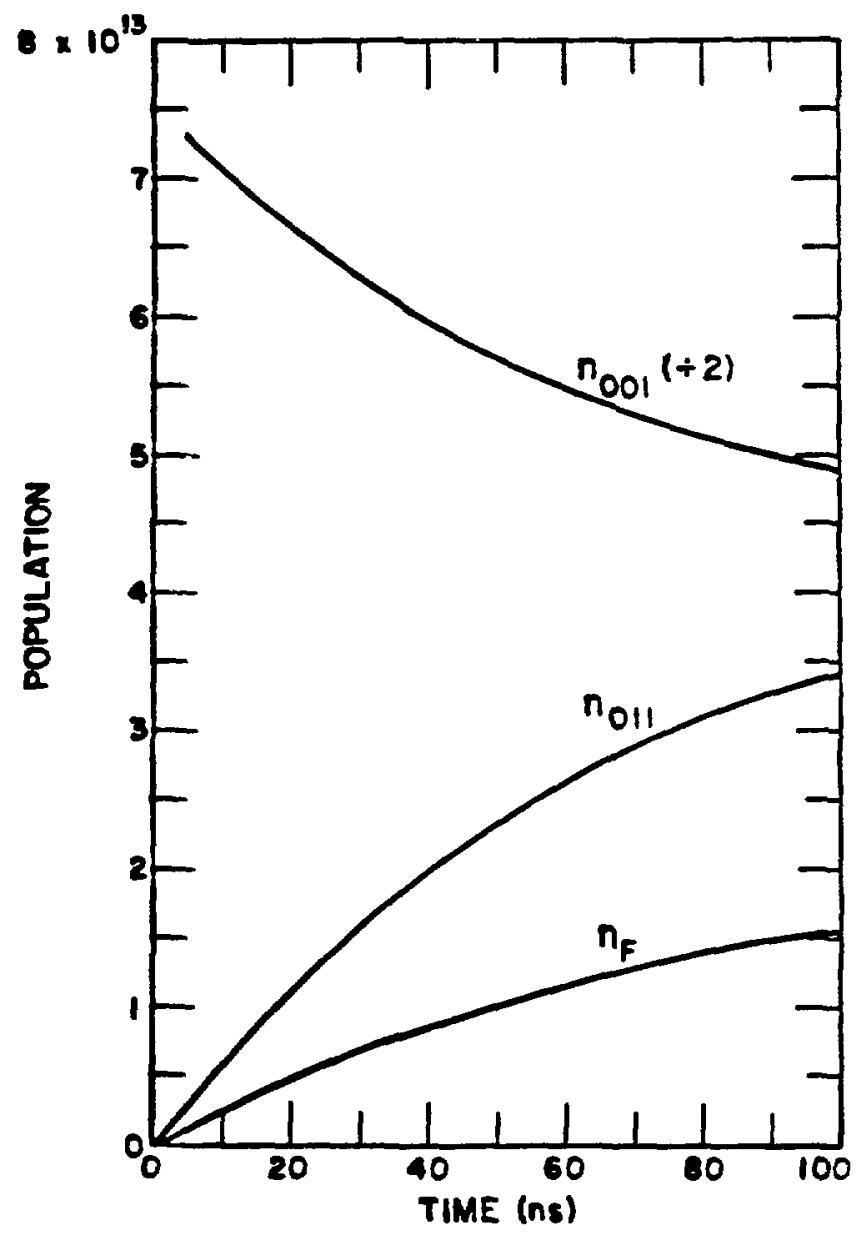

Figure III-4. The Three-Level Model Response

The behavior of $n_{001}, n_{011}$, and $n_{F}$ after a saturating pump pulse is shown (10 torr). The $n_{F}$ curve represents the density sum of $021+02^{2} 1+101$. 
by $n_{F}^{\star}$ is the sum of $021,02^{2} 1$, and 101 . The conditiuns for these simulations are $700^{\circ} \mathrm{K}$ and 10 torr. Each of these vibration states is seen to undergo a monotonic approach to some new quasi-equilibrium value. If the values of $n_{F}$ and $n_{01}$ are added at any time, the sum is found equal to the decrease in $n_{001}$ to within a few per cent. The difference indicates the small amount of population which is transferred to 002 .

With rotational equilibrium and constant lower levels assumed, a simple set of rate equations describes the behavior of $n_{001}, n_{F}$, and $n_{017}$. These are

$$
\begin{aligned}
& \dot{n}_{F}=-n_{F} N_{0} k_{F}+n_{001} N_{F} k_{F} \\
& \dot{n}_{011}=-n_{011} N_{0} k_{1}+n_{001} N_{1} k_{3} \\
& n_{001}=-n_{F}-n_{011}+n_{p}
\end{aligned}
$$

In these equations $n_{p}$ is the pumped density $\left\{n_{00}\right.$ at $\left.t=0\right)$ and $N_{0}, N_{1}$, and $N_{F}$ are $N_{000}, N_{010}$, and $N_{020}+N_{02}{ }^{2}+$ N100, respectively. These equations simply represent the important $v_{3}$ transfer rates and population conservation among the included levels. The forward and reverse rate coefficients are approximately equal, as shown, since

*The $F$ subscript means fermi group. The $10^{\circ} \mathrm{l}$ and $02^{\circ} 1$ states are heavily mixed like $10^{\circ} 0$ and $02^{\circ} 0$. 
$\Delta E \ll k_{B} T$. The first equation, for $n_{F}$, is really

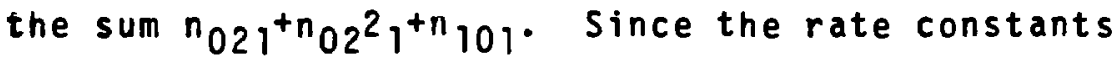
$\left(k_{F}\right)$ involved are assumed equal, they may be factored so the sums $n_{F}$ and $N_{F}$ result.

Substitution of the third expression gives

$$
\begin{aligned}
& \dot{n}_{F}=-n_{F}\left(N_{0}+N_{F}\right) k_{F}-n_{011} N_{F} k_{F}+n_{P} N_{F} k_{F} \\
& \dot{n}_{011}=-n_{011}\left(N_{0}+N_{1}\right) k_{1}-n_{F} N_{1} k_{1}+n_{P} N_{1} k_{1}
\end{aligned}
$$

By redifferentiation and appropriate substitutions, two uncoupled second-order equations may be obtained,

$$
\begin{aligned}
& \ddot{n}_{F}+\left[\left(N_{0}+N_{F}\right) k_{F}+\left(N_{0}+N_{1}\right) k_{1}\right] \dot{n}_{F}+\left(N_{0} N_{T} k_{F} k_{1}\right) n_{F} \\
& =n_{P} N_{F} N_{0} k_{F} k_{1} \\
& \ddot{n}_{011}+\left[\left(N_{0}+N_{F}\right) k_{F}+\left(N_{0}+N_{1}\right) k_{1}\right] \dot{n}_{011}+\left(N_{0} N_{T} k_{F} k_{1}\right) n_{011} \\
& =n_{D} N_{1} N_{0} k_{F} k_{1}
\end{aligned}
$$

with $N_{T}=N_{0}+N_{1}+N_{F}$. These levels apparently behave as identical damped oscillators with constant, but slightly different, driving forces which are turned on at $t=0$. The solutions have the form $Y(t)+F$, with $F$ the particular solution and $Y(t)$ the complementary solution, 
or the solution of the homogeneous equation. The particular solutions, for constant driving terms (Murphy, 60, p. 146), are $n_{p}\left(N_{F} / N_{T}\right)$ for the first equation and $n_{p}\left(N_{1} / N_{T}\right)$ for the second. The homogeneous solution has the form (Murphy, 60, P. 84)

$$
c_{1} e^{r} t^{t}+c_{2} e^{r_{2} t}
$$

in which $r_{1}$ and $r_{2}$ are the roots of the characteristic equation

$$
r^{2}+\left[\left(N_{O}+N_{F}\right) k_{F}+\left(N_{0}+N_{1}\right) k_{1}\right] r+N_{O} N_{T} k_{F} k_{l}=0
$$

The solution for $r$ is

$$
\begin{aligned}
r & =-\frac{1}{2}\left[\left(N_{0}+N_{F}\right) k_{F}+\left(N_{0}+N_{1}\right) k_{1}\right] \\
& \pm \frac{1}{2}\left\{\left[\left(N_{0}+N_{F}\right) k_{F}+\left(N_{0}+N_{1}\right) k_{1}\right]^{2}-4 N_{0} N_{T} k_{F} k_{1}\right\}^{\frac{3}{2}}
\end{aligned}
$$

With some manipulation the discriminant may be brought to the form

$$
\left[\left(N_{0}+N_{F}\right) k_{F}-\left(N_{0}+N_{1}\right) k_{1}\right]^{2}+4 N_{F} N_{1} k_{F} k_{1}
$$

This quantity is always positive, so $n_{F}$ and $n_{01}$ are overdamped. Thus monotonic responses are to be expected. 
If the substitutions

$$
\begin{array}{ll}
\Gamma=\frac{1}{2}\left[\left(N_{0}+N_{F}\right) k_{F}+\left(N_{0}+N_{1}\right) k_{1}\right] & \text { II I-23 } \\
\gamma=\frac{1}{2}\left\{\left[\left(N_{0}+N_{F}\right) k_{F}+\left(N_{O}+N_{1}\right) k_{1}\right]^{2}-4 N_{O} N_{T} k_{F} k_{1}\right\}^{\frac{3}{2}} & \text { II I }-24
\end{array}
$$

are made so that $r_{1}=-\Gamma-\gamma$ and $r_{2}=-\Gamma+\gamma$, and the initial conditions $n_{F}=n_{011}=0$ are used, the solutions which result are

$$
\begin{aligned}
n_{F} & =n_{p} \frac{N_{F}}{N_{T}}\left[1-\frac{\left(N_{T} k_{F}-\Gamma+\gamma\right)}{2 \gamma} e^{-(\Gamma+\gamma) t}\right. \\
& +\frac{\left(N_{T} k_{F}-\Gamma-\gamma\right)}{2 \gamma} e^{-(\Gamma-\gamma) t]} \\
n_{011} & =n_{p} \frac{N_{1}}{N_{T}}\left[1-\frac{\left(N_{T} k_{1}-\Gamma+\gamma\right)}{2 \gamma} e^{-(\Gamma+\gamma) t}\right. \\
& \left.+\frac{\left(N_{T} k_{T}-\Gamma-\gamma\right)}{2 \gamma} e^{-(\Gamma-\gamma) t}\right]
\end{aligned}
$$

The population conservation relation, Eq. III-18, then gives

$$
\begin{aligned}
n_{001}=n_{p} \frac{N_{0}}{N_{T}} & \left\{1+\frac{\left[N_{T}\left(N_{F} k_{f}+N_{1} k_{1}\right)-\left(N_{f}+N_{1}\right)(\Gamma-\gamma)\right]}{2 \gamma N_{0}} e^{-(\Gamma+\gamma) t}\right. \\
I I I-27 & -\frac{\left[N_{T}\left(N_{F} k_{F}+N_{j} k_{1}\right)-\left(N_{F}+N_{1}\right)(\Gamma+\gamma)\right]}{2 \gamma N_{0}} e^{-(\Gamma-\gamma) t\}}
\end{aligned}
$$


For times long after the pumping, the coefficients

(outside the braces) imply the new equilibrium conditions, $n_{001}^{e}: n_{011}^{e}: n_{F}^{e}:: N_{0}: N_{1}: N_{F}$. Thus the three-level system, with total density $n_{p}$, reaches a temporary equilibrium at the gas temperature with $n_{00}$ playing the role of the ground state.

Interpretation of sequence-band data requires the individual level $n_{10}$ of the $n_{F}$ group. A. reasonable estimate for this state would be to give it the $n_{F}$ time dependence but modify the amplitude coefficient to give the appropriate equilibrium density. Thus

$$
\begin{aligned}
n_{101} & =n_{p} \frac{N_{100}}{N_{T}}\left[1-\frac{\left(N_{T} k_{F}-\Gamma+\gamma\right)}{2 \gamma} e^{-(\Gamma+\gamma) t}\right. \\
& \left.+\frac{\left(N_{T} k_{F}-\Gamma-\gamma\right)}{2 \gamma} \in-(\Gamma-\gamma) t\right]
\end{aligned}
$$

Comparison of these expressions with the multilevel program output shows very good agreement if amplitude adjustments are allowed (by $n_{p}$ variations). It was also found that an origin shift of 1 to 2 ns was required to really match the curves in the comparison. This is probably needed because pumping and rotational relaxation are not included in the analytic model. It is remarkable that such simple relations are useful in what orlginally appeared to be a complex system. 
3. The $n_{002}$ Solution

The rate equation for the $v_{3}$ transfer $001 \rightarrow$

002 is

$$
\dot{N}_{002}=-N_{002} N_{0} k_{2}+N_{001}^{2} k_{2}
$$

in which $k_{2}=k_{002}$. If the assumption is made that

this reaction has negligible influence on 001 density, the equation can be solved, given $n_{001}(t)$. Care must be taken in converting to an $n_{02}$ equation because of the nonlinear coupling to 001 . The equilibrium condition is

$$
N_{002}^{e} N_{0}^{e}=N_{001}^{e} N_{001}^{e}
$$

With the substitutions $N_{002}=N_{002}^{e}+n_{002}(t)$ and $N_{001}=N_{001}^{e}+n_{001}(t)$, Eq. III-29 becomes

$$
\dot{n}_{002}=-N_{002}^{e} N_{0} k_{2}-n_{002} N_{0} k_{2}+\left(N_{001}^{e}\right)^{2} k_{2}+2 N_{001}^{e} n_{001} k_{2}+n_{001}^{2} k_{2}
$$

The first and third terms cancel because of the equiliorium condition. In the present experiment $n_{001}$ is expected to be a substantial fraction of $N_{00}$ so the last term must be retained. Thus

$$
\dot{n}_{002}+n_{002} N_{0} k_{2}=\left(2 N_{001}^{e} n_{001}+n_{001}^{2}\right) k_{2}
$$


With the definition

$$
u=n_{002} e^{N_{0} k_{2} t}
$$

one has

$$
\begin{aligned}
\dot{u} & =\left(\dot{n}_{002}+N_{0} k_{2} n_{002}\right) e^{N_{0} k_{2} t} \\
& =\left(2 N_{001}^{e} n_{001} k_{2}+n_{001}^{2} k_{2}\right) e^{N_{0} k_{2} t}
\end{aligned}
$$

in which Eq. III-30 has been used. Then since

$$
u=n_{002} e^{N_{0} k_{2} t}=\int \dot{u} d t+c
$$

the solution for $n_{002}$ is

$$
\begin{aligned}
& n_{002}=c e^{-N_{0} k_{2} t}+e^{-N_{0} k_{2} t} k_{2} \\
& x S\left(2 N_{001}^{e} n_{001}+n_{001}^{2}\right) e^{N_{0} k_{2} t} d t
\end{aligned}
$$

From Eq. III -27

$$
n_{001}=n_{p} \frac{N_{0}}{N_{T}}\left[1-A e^{-(\Gamma-Y) t}+B e^{-(\Gamma+Y) t}\right] \quad \text { III-32 }
$$

with

$$
A=\frac{N_{T}\left(N_{1} k_{1}+N_{F} k_{F}\right)-\left(N_{1}+N_{F}\right)(\Gamma+Y)}{2 Y N_{0}}
$$




$$
B=\frac{N_{T}\left(N_{j} k_{j}+N_{F} k_{F}\right)-\left(N_{\eta}+N_{F}\right)(\Gamma-Y)}{2 \gamma N_{0}}
$$

Straightforward integration leads to

$$
\begin{aligned}
n_{002} & =\frac{n_{p}}{N_{T}}\left(2 N_{001}^{e}+n_{p} \frac{N_{0}}{N_{T}}\right)\left(1-e^{N_{0} k_{2} t}\right) \\
& -\frac{2 k_{2}\left(n_{p} N_{0} / N_{T}\right) A}{N_{0} k_{2}-(\Gamma-\gamma)}\left(N_{001}^{e}+n_{p} \frac{N_{0}}{N_{T}}\right)\left(e^{-(\Gamma-\gamma) t}-e^{-N_{0} k_{2} t}\right) \\
& +\frac{2 k_{2}\left(n_{p} N_{0} / N_{T}\right) B}{N_{0} k_{2}-(\Gamma+\gamma)}\left(N_{001}^{e}+n_{p} \frac{N_{0}}{N_{T}}\right)\left(e^{-(\Gamma+\gamma) t}-e^{-N_{0} k_{2} t}\right) \\
& +\frac{k_{2}\left(n_{p} N_{0} / N_{T}\right)^{2} A^{2}}{N_{0} k_{2}-2(\Gamma-\gamma)}\left(e^{-2(\Gamma-\gamma) t}-e^{-N_{0} k_{2} t}\right) \\
& +\frac{k_{2}\left(n_{p} N_{0} / N_{T}\right)^{2} B}{N_{0} k_{2}-2(\Gamma+\gamma)}\left(e^{-2(\Gamma-\gamma) t}-e^{-N_{0} k_{2} t}\right) \\
& -\frac{k_{2}\left(n_{p} N_{0} / N_{T}\right)^{2} 2 A B}{N_{0} k_{2}-2 \Gamma}\left(e^{-2 \Gamma t}-e^{-N_{0} k_{2} t}\right)
\end{aligned}
$$

The solution is seen to be a sum of exponentials like the solutions for the other levels. Numerical studies showed agreement with the computer solution and also showed that the first and third terms are the most important.

4. Sequence-band Study

The determination of $k_{002}$ by sequence-band (002-101) probe suffers from a complication which makes the signals difficult to interpret. Because the probe 
absorption is governed by the quantity $n_{101}-n_{002}$, a change in either level affects the signal. However, if the $n_{002}$ transient were dominant, the data would still be expected to be sensitive to the $k_{002}$ value. Unfortunately, the reverse is true; $n_{101}$ receives considerably more of the 001 excess than 002. This situation is illustrated in Fig. III-5 which shows computer output of $n_{002}$ and $n_{101}$ versus time at 10 torr. The ${ }^{10} 10$ curve shows the overdamped exponential rise expected from Eq. III-28. The $n_{002}$ result shows it is not only smaller, but in addition it displays a faster risetime and a slight overshoot. The analytic solution shows the same behavior.

During the $n_{002}$ rise the signal should be most sensitive to $k_{002}$. The rest of the sequence-band signal should be quite sensitive to $k_{101}$, thus providing a means for its measurement. Examination of the figure (III-5) for $t<40 \mathrm{~ns}$ reveals an important effect. The $n_{002}$ value actually exceeds $n_{101}$ during the first 20 ns. A signal proportional to the transmission would be positive for the first 20 ns then would have a zero crossing and would be negative thereafter. Crossover times are easy to determine on scope traces, so a further model study of the phenomenon was undertaken. 


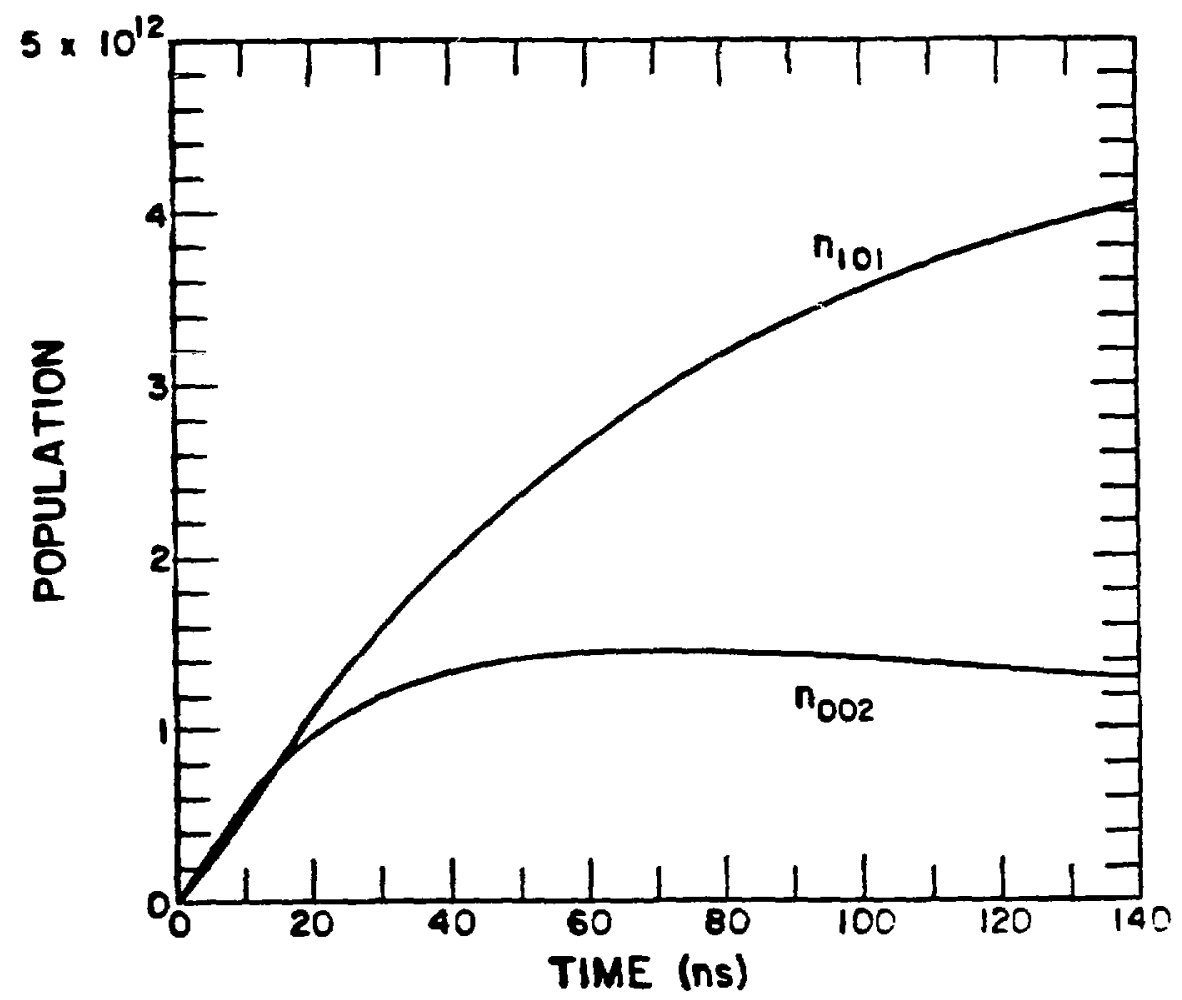

Figure III-5. Response of the Sequence Levels

The predicted behavior of $n_{002}$ and $n_{101}$ after the pump pulse is shown ( 10 torr). The sequence-band signal goes like the difference between the two. 
The results are shown in Fig. III-6. The curves each show predicted values of the signal for various conditions of interest. Curve a is for the usual case of $k_{002}=6$ $\times 10^{6}$. Curves $b$ and $c$ result from halving and doubling the value, respectively. The obvious conclusion is the crossover time is extremely sensitive to the desired rate constant. Curve d shows the result of doubling the pressure (20 torr). Reduction of the pump rate in the code showed the amplitude of the bump feature to be reduced also (for nonsaturating intensities), but the crossover time was unaffected.

The bump would be a very attractive feature to look for, in the $1 a b$, except for one thing; the estimated signal strength is very weak. A typical noise value at the scope is $5 \mathrm{mV}$. The simulation which gave the estimate in the figure for the signal was for laboratory conditions, of the lasers and detection system, which had already been achieved. These conditions appeared more than sufficient for the rotational relaxation measurements and also for determining $k_{011}$ and $k_{101}$. However, a good measurement of $\mathrm{K}_{\mathrm{OO} 2}$ appeared likely to require more detector sensitivity, more probe laser power, and also amplification and bandwidth games. 


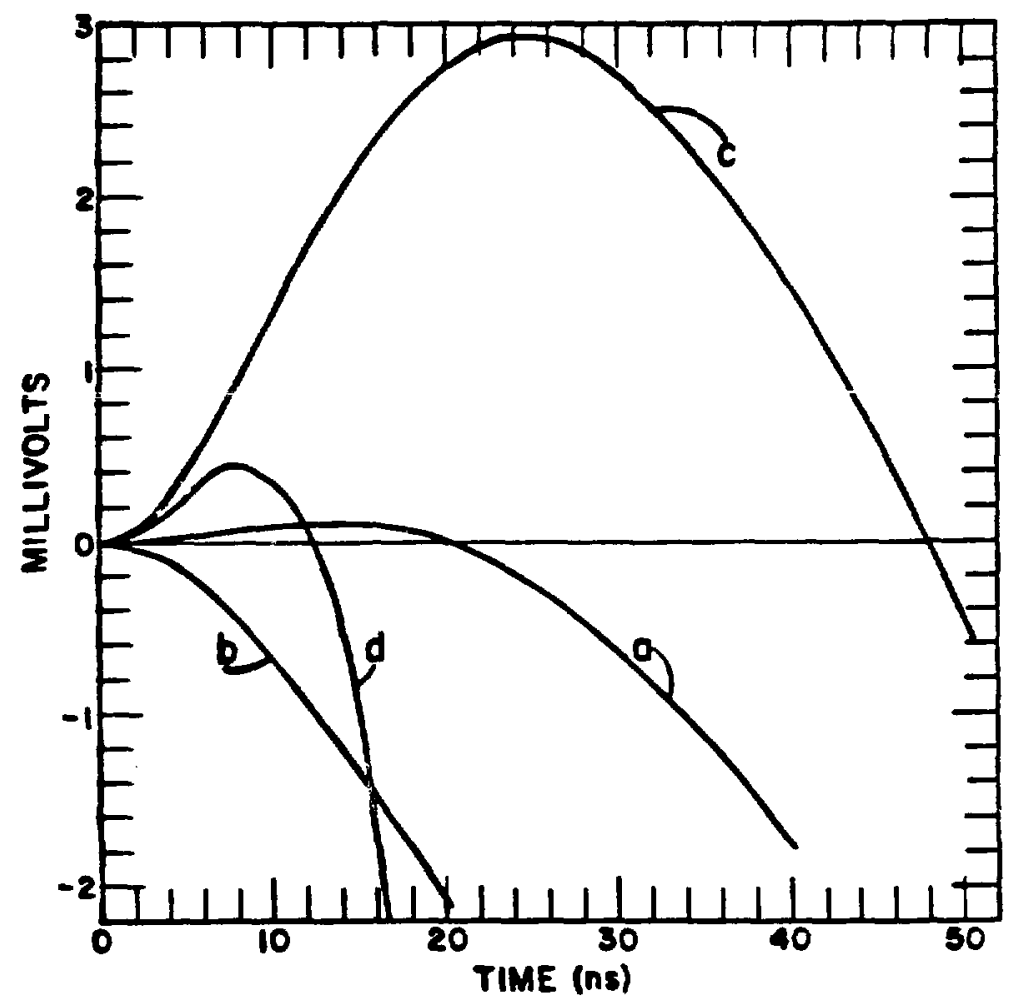

Figure III-6. The Sequence-Band Study

The results of the study of the sequence-band bump feature are shown. The four plots are for: (a) $k=6 \times 10^{6}$ torr ${ }^{-1} \sec ^{-1}, p=10$ torr; (b) $k=3 \times 10^{6}, p=10$; (c) $k=12 \times 10^{6}, p=10$, and (d) $k=6 \times 10^{6}, p=20$. 


\section{CHAPTER IV}

\section{EXPERIMENTAL APPARATUS AND PROCEDURES}

In this chapter the details of the experimental work are described. Included are descriptions of the equipment, the procedures, and the data reduction techniques.

Essentially the same apparatus is used for the $R-R$ and the $V-V$ measurements. Only minor modifications are required to change from one to the other. Thus the following sections generally apply to both experiments.

\section{A. Experimental Apparatus}

The double resonance measurements have required a system composed of two $\mathrm{CO}_{2}$ lasers, the pump and the probe. Also utilized are an electrooptic shutter to limit the pump pulse to $4 \mathrm{~ns}$ and a heated absorption cell. These items are shown in a system diagram in Fig. IV-l. At the top, the probe laser is shown to consist of a laser tube with Brewster windows, a grating, G, and an output coupler, $O C$. The element $H C$ is an intracavity hot cell used for sequence-band lasing. The pump laser is a high-power TEA laser (transverse discharge, electrically 


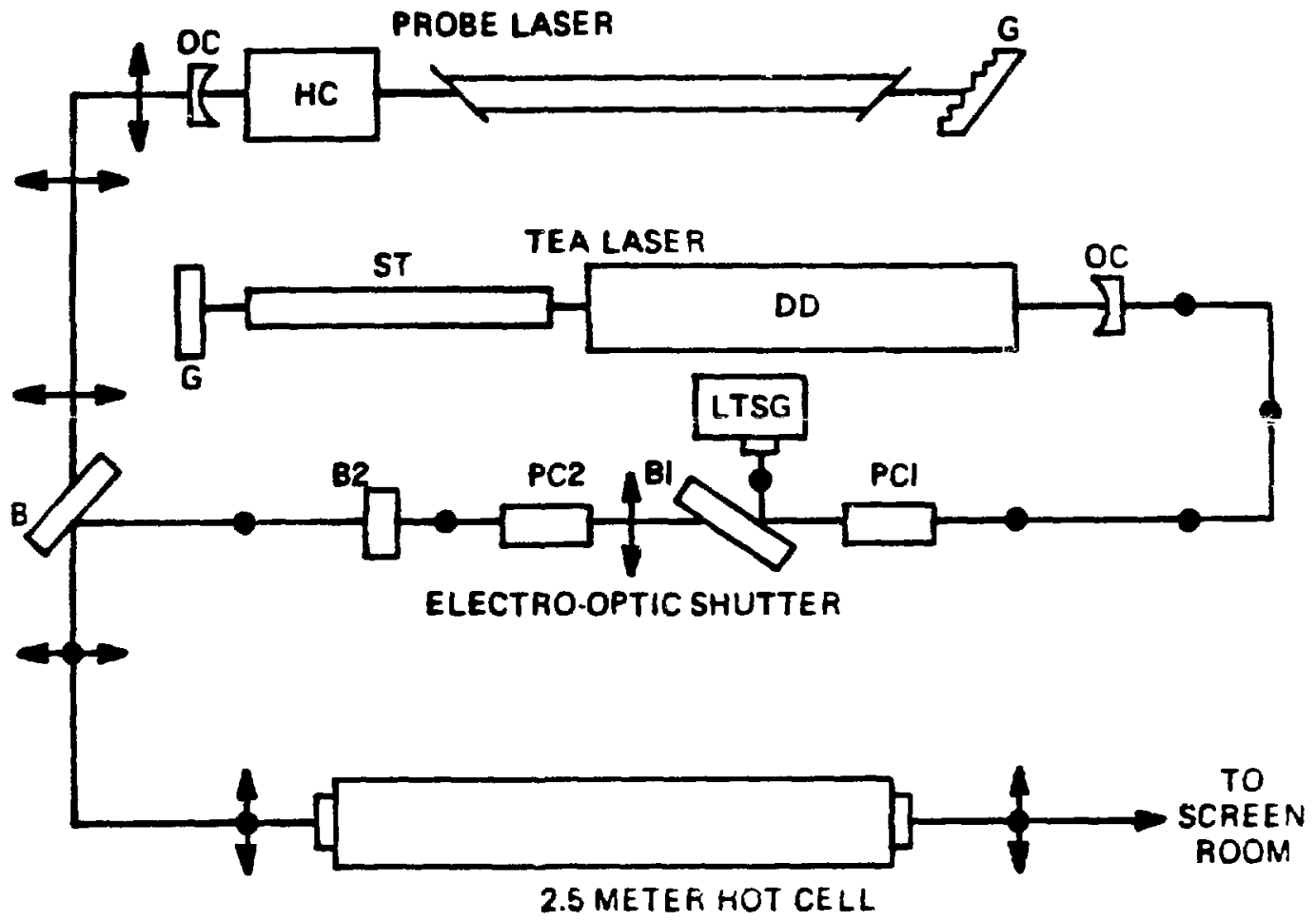

Figure IV-1. The Double Resonance System

The equipment shown consists of the probe laser, the TEA laser with an electrooptic shutter, and the heated absorption cell. 
excited, atmospheric pressure). The item DD represents the double-discharge device which is the high-gain element of the oscillator cavity. The smoothing tube, ST, forces single mode oscillation which results in a temporally smooth output pulse. Brewster angle windows are also utilized in the cavity, so the output is polarized. The two lasers have opposite polarizations; the probe is vertical, the pump horizontal. These lasers are described in greater detail in Section IV.C.

The 300-mJ output of the TEA laser is in the form of a half microsecond pulse. The $4-n s$ pulse, required for the experiment, is gated out of the long pulse by an electrooptic shutter (McLellan and Figuaira, 79). This device has three types of elements: two Pockels cells, PCl and PC2; two Brewster angle polarizers, $B 1$ and $B 2$; and a laser triggered spark gap, LTSG. By voltage pulse actuation, a Pockels cell can rotate the beam polarization by $90^{\circ}$. The element $B l$ only passes vertical polarization. Basically the shutter works by switching the horizontal polarization to vertical for 4 ns.

The initiation time of the voltage switch is determined by the LTSG. The leading edge of the incident light pulse is reflected from $B 1$ into LTSG causing the gap to break down. This initiates the 4-ns voltage switching of $P C 1$, thus the device is seen to be self triggering. 
The shutter consists of two stages for a better on/off extinction ratio. The end result is a $1-\mathrm{mJ}$, horizontaliy polarized, 4-ns pulse.

The electrical detalis of the shutter are indicated in Fig. IV-2. The solid lines are electrical pathways; the optical paths are indicated as dashed lines. The high voltage, +HV, charges the $2-n s, 50-\Omega$ charge line, $C L$, through a high resistance, $R$. The rest of the circuit, consisting of LTSG, PC), PC2, and HVT (high-voltage terminator), is also designed to approximate a $50-\Omega$ transmission-line system. When the leading edge of the incident light pulse is directed into the gap of LTSG, the gap breaks down shorting $C L$ to the rest of the line. $C L$ discharges, for twice its transit time, sending a 4-ns square voltage pulse of half the charge voltage down the line. This causes $P C 1$ to rotate the polarization of the light for $4 \mathrm{~ns}$. This vertically polarized light is transmitted through $B$ and is reswitched to horizontal polarization at PC2. The electrical line between $\mathrm{PCl}$ and PC2 has the appropriate length so the light and voltage arrive at PC2 simultaneously. The electrical pulse experiences a 50- $\Omega$ termination at HVT. The terminator also has a low voltage pickoff which furnishes a very conventent scope trigger.

The cross hatched portions of $P C 1$ and $P C 2$ are CdTe 


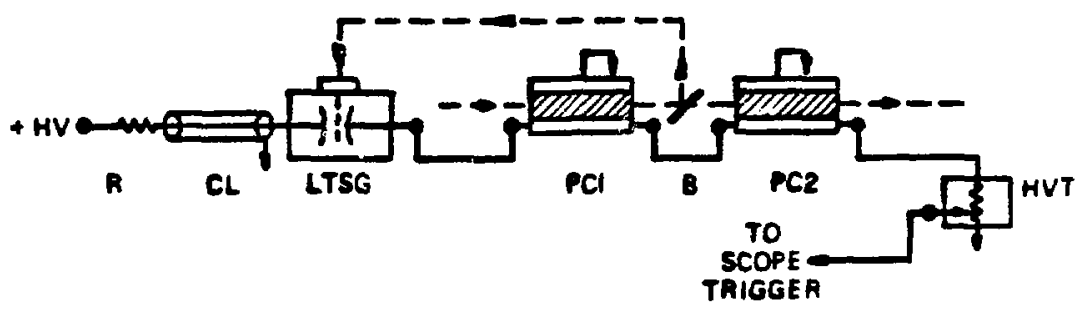

Figure IV-2. Electrical Diagram of the Shutter

The two Pockels cells are actuated by a high-voltage pulse which propagates along the 50- $\Omega$ system. The self-triggering of the system is due to the lasertriggered spark gap (LTSG). 
crystals. These elements are about $8 \mathrm{~mm}$ square by $40 \mathrm{~mm}$ in length and have a half-wave voltage of about $8 \mathrm{kV}$. The two-stage system consisting of these crystals and stacked germanium Brewster windows gave a measured extinction ratio of about $10^{-5}$.

Figure IV-I shows that the output of the shutter goes to another Brewster plate where the beams are joined. Here, if the beams approach each other at $30^{\circ}$ angle (not shown), advantage may be taken of the Ge Brewster angle properties, so that one beam is completely transmitted and 75\% of the other beam is reflected in their joining.

As depicted in the figure the joined beam paths continue to the heated absorption cell. This cell was designed and constructed (Tercovich, 78) to provide a $250 \mathrm{~cm}$ absorption length with excellent temperature uniformity. The entrance and exit ports have a diameter of $15 \mathrm{~cm}$ with Brewster-angle $\mathrm{NaCl}$ windows. The temperature was set at $427^{\circ} \mathrm{C}\left(700^{\circ} \mathrm{K}\right)$ for the experiment and was stable within the accuracy of the thermocouple sensor gauges.

The transmitted beams exit the cell and are then directed into the screen room to the signal detection system. 
B. Signal Detection

The screen room prevents electrical noise, generated by the high-voltage switching associated with the lasers, from interfering with the measurements. A schematic diagram of the optics and electronics, housed in the screen room, is shown in Fig. IV-3. The function of this apparatus is to eliminate the pump pulse from the beam path and detect the signal riding on the probe pulse.

The light pulses entering the enclosure are shown in the upper left corner of the figure. The pump pulse typically would be a square, 4-ns, half-MW pulse of $9-\mu m$ light. The probe, then, might be a $10-\mu \mathrm{m}$, 1 Watt, 6+10-ms pulse with the much weaker signal of interest superimposed on it, as fllustrated in the figure. As shown also, the two beams are cross polarized.

The first element encountered is an analyzer composed of four ZnSe plates and four Ge plates. These plates present Brewster angle surfaces to the light, and the horizontally polarized pump undergoes a substantial reflection at each interface ( $75 \%$ for GE, $50 \%$ for $2 n S e$ ). The pump intensity is reduced to the same order as the probe, so it can then be subjected to wavelength discrimination in the next element, a low-resolution grating monochomator. The pump pulse is essentially eliminated by this system while the probe beam is transmitted and directed to the Ge:Hg detector. 


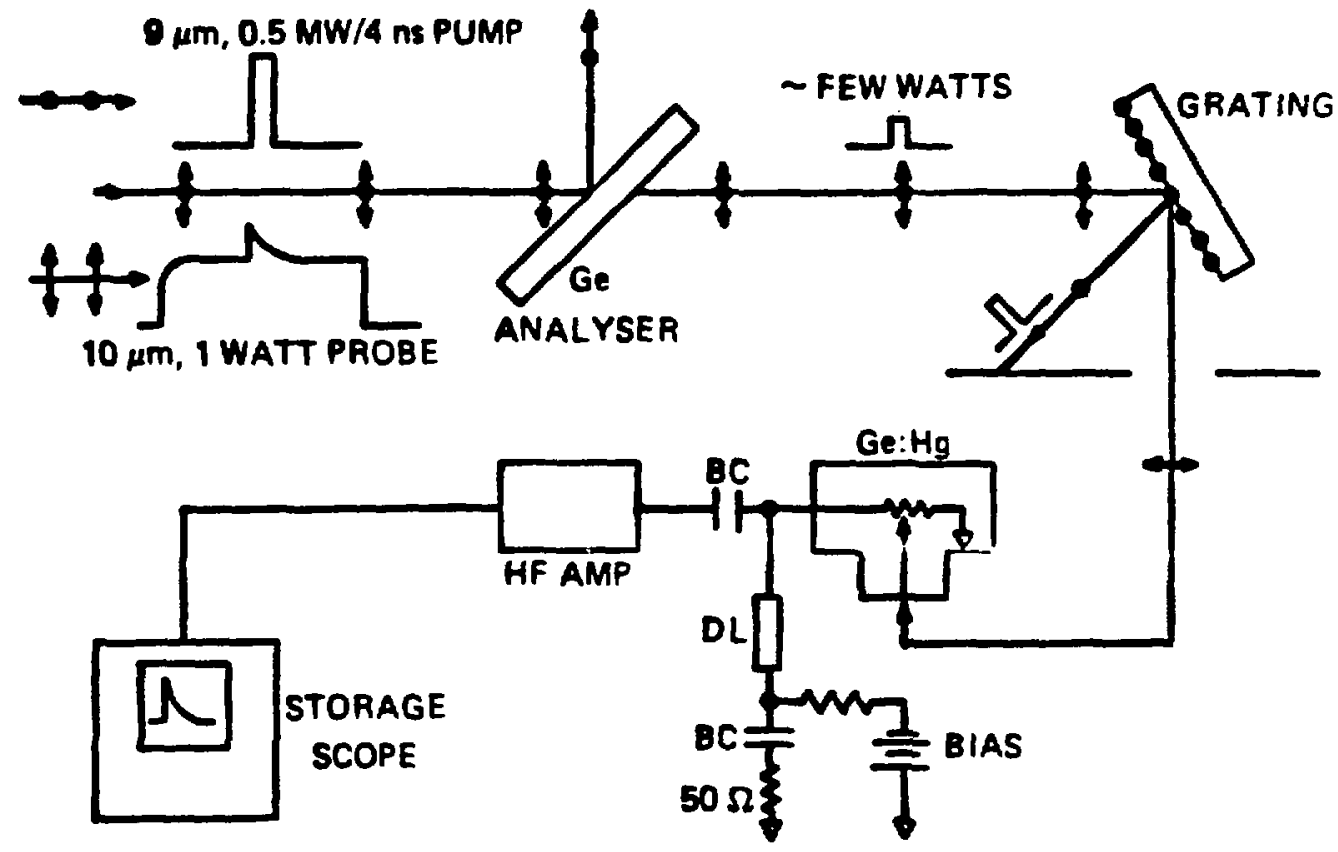

Figure IV-3. Screen-Room Optics and Electronics

The function of the optical elements and the electronics in the screen room is to eliminate the pump pulse from the beam path and to detect the signal riding on the probe pulse. 
The detector apparatus is comprised of four parts. These are the detector, the bias supply, the blocking capacitor, and the preamp.

The detector is a commercial $\mathrm{Ge}: \mathrm{Hg}$ photoconductor from Santa Barbara Research Corp. (Model 9145-2HS). The photoconducting element is housed in a liquid helium dewar. Measurements on the 4-ns pulse have indicated the detector has a subnanosecond risetime and about a 3-ns falltime. The linearity of the detector, at the appropriate bias, was checked and found to be adequate for the signal levels expected.

The bias roltage and blocking capacitor network is a conceptually simple requirement. The bias supply draws current through the detector element ( $2 \mathrm{M} \Omega$ at $4^{\circ} \mathrm{K}$ ) creating a voltage drop essentially equal to the bias voltage. The blocking capacitor keeps the dc voltage from the amplifier and scope but passes transients created by light pulses which reach the photoconductive element.

In the present case, the constraints imposed by the signals to be measured did require some ingenuity in the biasing. For one thing weak signals require the highest possible bias value which is about 200 volts. Beyond this voltage, low-level microsecond oscillations are produced which probabiy indicate breakdown at the element. The bandwidth is another important biasing concern. The probe 
output pulses which determine baseline transmission need good low frequency response while the common upper level data, in some cases, may make a few hundred MHz desirable. Also the high frequencies associated with the fast rise on the common upper-level data are reflected from every point in the circuit which does not have a 50- $\Omega$ impedance.

After many iterations a scheme was developed which was adequate electrically and stili simple enough to live with. Electrolytic capacitors seem made for this application as blocking capacitors. They provide high capacitance and high voltage standoff in a small package. The fact that single polarity voltage is required is no detriment since the bias is always greater than the signal. The blocking capacitor, BC, was rated at $10 \mu \mathrm{F}$ and $450 \mathrm{VDC}$. It was fitted into a small electronics box, so it would have good transmission properties at high frequencies. The response was adequate for the frequency range of interest.

The electrical reflections from the bias box were particularly troublesome. The arrangement which eliminated this problem is shown in Fig. IV-3. A 50splitter was placed at the detector. A $50-\Omega$ delay line of over $200 \mathrm{~ns}$ is shown as DL. This leads to the shielded bias box. The bias was provided by two $90-v$ batteries in most cases. The connection to the $50-\Omega$ line is through 
a 10-k $\Omega$ resistor which presents a high impedance for the battery branch. It also serves as a current limiter in case the detector warms to room temperature and becomes conducting. The second branch in the bias box consists of an electrolytic blockings capacitor, $B C$, and a $50-\Omega$ termination. The strategy was to make the bias arm look like a 50- $\Omega$ system to the signals, so no reflections would be produced there. This system was used for both high frequency and low frequency measurements, and high frequency reflections were greatly reduced. In addition persistent reflections were delayed by $\mathrm{DL}$ sufficiently long so they would not appear on the scope trace.

The electrical signal next encounters the high frequency preamp, HF AMP. Several models with $175 \mathrm{MHz}$ to 1.3 GHz bandwidth were used. The only real problem was in their low-end falloff characteristics. One function the preamp must perform is to transmit only the signal portion of the several millisecond probe pulse. Units with fair low-frequency response invariably are affested by the 6-ms probe pulse which causes the scope baseline to jump. This is an intolerable annoyance. On the other hand, the low-end cutoff must not be so high that flat portions of the signals are caused to droop.

The storage scope used for all data collection was a 400-MHz Tektronix 7834. The high and low-frequency pulses 
could usually be accommodated by the 350-MHz $7 A 24$ plug-in module. The low frequency measurements of $V_{B}$ were made without the preamp, so the low end cutoff was determined by the blocking capacitor at the detector in this case.

C. The $\mathrm{CO}_{2}$ Lasers

In Section IV.A. the laser cavities were briefly described. In the following paragraphs the details of the two lasers are given. In addition the techniques required for sequence-band $(002+101)$ lasing and hot-band $(011+110)$ lasing, by the probe laser, are discussed.

The pump laser consists of a high-gain, pulsed, double-discharge element and a low-pressure, dc-discharge tube and associated optics as shown in Fig. IV-l.

High gain is produced in a pulsed 55-kV discharge between two Rogowski electrodes $85 \mathrm{~cm}$ in length and separated by a few centimeters. The laser gas is a He- $\mathrm{CO}_{2}-\mathrm{N}_{2}$ mix at atmospheric pressure. Stability of the discharge is assured by uv preionization. The source is a 25-kV s rk-iine, or flashboard, located a few centimeters from the discharge volume and extending along the full length of the Rogowski electrodes.

The laser gas, being at atmospheric pressure, has a propagation linewidth of a few gigahertz. This bandwidth allows longftudinal mode spikes to form on the output 
pulse. These spikes are, in general, undesirable. The smoothing tube is a low-pressure, longitudinal discharge device with a 50-MHz baridwidth which acts as a narrow-band filter. This permits lasing on a single iongitudinal mode only. The combination of these two gain elements results in a temporally smocih high-energy output pulse.

The output coupler normally used was a Ge mirror with $80 \%$ reflectivity on the cavity side and AR coated for $10 \mu \mathrm{m}$ on the opposite side. At the other end of the cavity is a Littrow grating blazed for $10 \mu \mathrm{m}$ operation. The output was always a single rotational line. The $80-\mathrm{cm}$ smoothing tube also lases by itself with a continuous output. This mode of operation is indispensible for cavity tuning and pump beam alignment. The lossy cavity made cw lasing difficult on the weaker 9- $\mu$ m transitions. This annoying situatior was remedied by cooling the tube walls by flowing refrigerated methanol through the cooling jackets. The resulting wall temperature was less than $0^{\circ} \mathrm{C}$ as evidenced by ice buildup on the laser tube (which necessitated occasional defrosting). The smoothing tube could put out a couple of watts on strong lines.

The output of the pump is usually apertured down to give about $300 \mathrm{~mJ}$ in few-hundred nanosecond pulses. Damage threshold for the CdTe crystals is $10-20 \mathrm{MH} / \mathrm{cm}^{2}$. 
This is the factor which determines maximum allowable output for the lasers. The net output, after the shutter, was nominally a 1-mJ, 4-ns pulse. The oscillator could operate on 1 ines $P(10)_{10}-P(34)_{10}$ and a few of the 9-um lines.

The preceding few paragraphs describe the pump laser. The second $\mathrm{CO}_{2}$ laser was a low-power device which provided the probe beam. Several design variations were used during the early part of the experiment starting with a dc device similar to the smoothing tube. The insatiable need for higher and higher gain, especially for hot-band lasing, resulted in several changes in tube lengths, cooling schemes, and power supplies. The ultimate solution was to pulse the discharge for several milliseconds. This is accomplished by shorting the center electrode of the three-electrode 135-cm tube through a high-voltage vacuum tube (4PR60) with high voltage applied to the two end electrodes ( $15 \mathrm{kV}$ ). Ballast resistance of a few hundred $k \Omega$ limits the current through the tube. Puising the discharge in this manner resulted in a remarkable improvement ir lasing threshold. Cooling the tube was not required even when operating at $10 \mathrm{~Hz}$ which was necessary for alignment procedures.

The probe cavity grating is blazed for 11.6 um while the normal output coupler is a $96 \%$ reflector at $11 \mu \mathrm{m}$. 
Also in the cavity is the $75-\mathrm{cm}$ hot cell which is required for sequence-band lasing. This unit is a shortened replica of the 2.5-meter hot cell, and it also is normally kept at $700^{\circ} \mathrm{K}$. Absorption of the conventional $10-\mu \mathrm{m}$ lines is controlled by the pressure of $\mathrm{CO}_{2}$ to which the cell is filled. For normal and hot-band lasing the cell is simpiy evacuated.

The capabilities of the final version of this laser were never really investigated. By the time its evolution had reached the final stages, the impetus was only toward maximum sequence-band power. The interest in determining how many lines would lase had completely waned.

Part of the need for a high-performance probe laser is to provide lasing on the sequence-band transitions (Reid and Siemsen, 77). The strength of the gain coefficient, relative to 001 , may be estimated as the $002 / 001$ population ratio times a factor of two for the increased 002 transition moment. Since typical $v_{3}$-vibrational temperatures in laser discharges are $1500^{\circ} \mathrm{K}$ to $2000^{\circ} \mathrm{K}$, this gain ratio is about 0.3 . This means the gain is high and the transition shouid lase readily. The problem is the conventional bands overlap the sequence band with a wavelength shift of only a fraction of a micron. The result is the conventional lines with higher gain dominate the lasing action. Reid and Siemsen devised the technique 
of placing a cell of heated $\mathrm{OO}_{2}$ into the cavity as a selective loss element for the normal transitions. The sequence lines then have the higher net cavity gain and lase preferentially. This intracavity hot cell approach has been adopted in the present work and the sequence-band transitions have indeed been found to lase very well.

There is, however, a slight practical problem with hot-ceil lasers. The heated gas introduces some beam steering, so the cavity mirror settings required are quite different from those with the hot cell evacuated. The solution begins with the cavity optimized for conventional lasing and the cell evacuated. The hot- $\mathrm{CO}_{2}$ pressure is then increased by a few torr and then the cavity is readjusted. The procedure is iterated several times until the regular line is absorbed so badly it is near threshold. At this point the grating of the aligned cavity can be rotated slightly and the nearest sequence line should lase easily. Final pressures of 20 to 40 torr in the $70-\mathrm{cm}$ cell were appropriate at $400^{\circ} \mathrm{C}$.

The hot-band line: (Whitford et al., 77) have a still lower gain coefficient. The gain ratio $011 / 001$ involves a Boltzmann factor with the eriergy difference of the levels and the bending mode temperature. In typical discharges this temperature may be $2400^{\circ} \mathrm{K}$ which results in the gain coefficient ratio being $\sim 0.09$. Several cavity 
schemes netted only marginal lasing. The previously discussed pulsing of the discharge was then tried with very satisfying results.

Several 11-um lines were found to lase with this probe configuration. One line, $P(23)_{11}$, lased particularly well because it has a 42-MHz coincidental overlap with $P(56) 10$ (Reid and Siemsen, 73). This line must be avoided in the double resonance measurements, but its strength makes it useful for cavity optimization, beam alignment, and detection system alignment.

During the experiment the firing of the two lasers must be synchronized so the pump pulse occurs at the appropriate instant during the probe pulse. A schematic diagram of the electronics which accomplishes this timing and firing is shown in Fig. IV - 4. The sequence begins at the upper left. A momentary closure actuates the initial trigger generator, TG. The output is split with part going to the TEA laser and part to the pulse generator, $P G$. The output of this element causes the grid of the vacuum tube pulser, VP, to be grounded which essentially shorts one of the probe tube electrodes to ground. The duration of the laser output is controlled by the pulse generator. The pulse was usually 6 to $10 \mathrm{milliseconds.}$

The voltage pulse to the TEA laser is delayed for a few milliseconds by a delay generator, DG. Its output 


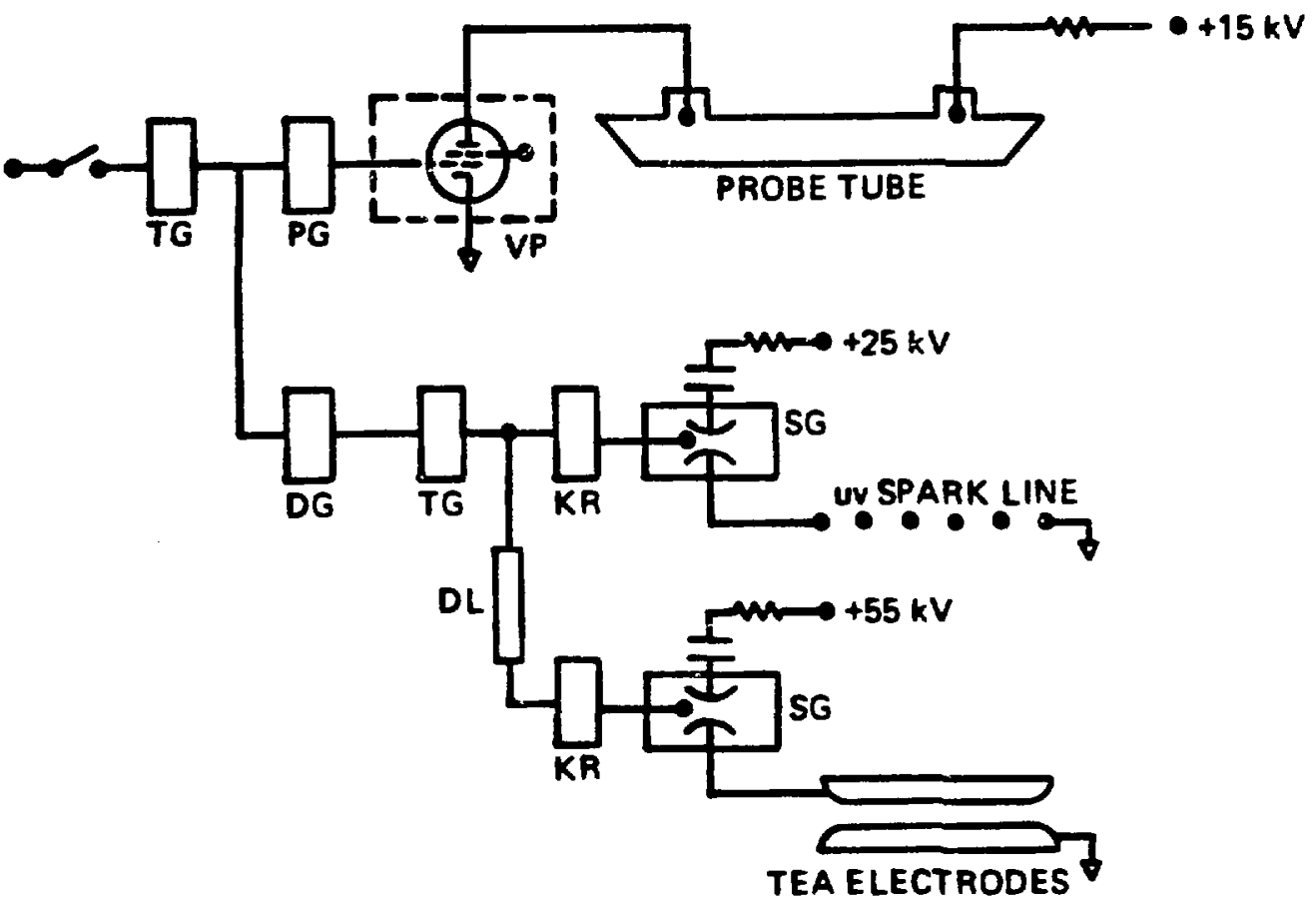

Figure IV-4. Laser Timing and Firing Diagram

A block diagram of the electronics which synchronizes the firing of the rasers is shown. A detailed description may be found in the text. 
actuates the pump trigger generator. This trigger is also split with part going to the main electrode gap and part to the uv preionizer. This uv trigger fires a krytron, $K R$, which generates a pulse with sufficient voltage to trigger the preionizer spark gap, SG. This shorts the 25-kV capacitor to the spark line. The ensuing breakdown between each of the isolated electrodes along this line causes a cascading spark to travel the length of the line. This floods the discharge volume with ionizing uv radiation. The trigger to the TEA electrodes is delayed a few microseconds and then a similar spark gap process causes a 55-kV breakdown across the Rowgowski electrodes. The preionized breakdown results in a uniform, arc-free, high-pressure discharge across the laser gas volume.

D. Experimental Procedures

Data taking sessions usually consisted of a series of many shots which produced storage scope traces which could be studied or photographed for later digitization and analysis. Each shot might test the effect of the variation of some gas parameter such as pressure or perhaps the change of a laser parameter. The following paragraphs describe first the general preparations for these sessions and then the detafls of each of the measurements. 
Several tasks had to be performed to prepare the equipment for data collection. These were very similar for each of the experiments. The lasers were found to require only minor adjustments each day if they were alloued to stabilize by operating for about an hour. This was usualiy the first step in the process. The time involved was not lost as there were other things to be done such as cooling the detector. This process began with a cool-down of the dewar by a filling with liquid nitrogen. When the rest of the system reached the point at which the detector was needed, the liquid nitrogen was emptied, and the liquid helium was transferred into the dewar from a large storage dewar.

Alignment of the beams, so they overlapped and hit the detector, was necessary each time the system was used and had to be checked each time a laser parameter was changed. The alignment was accomplished in two phases. First a red HeNe laser beam was made collinear with the probe beam, and the pair were directed through the system into the screen room. Once the screen room optics were adjusted for optimum probe signal into the Ge:Hg detector, the second phase began. For this, a second red beam was aligned to be collinear with the smoothing tube laser. The two were then directed to the electrooptic shutter. Half-wave plates, before the crystals, permitted the $\mathrm{CO}_{2}$ 
beam to be transmitted; the red beam was stopped. Two mirrors prior to the Brewster angle beam joiner were then adjusted to make the smoothing-tube beam collinear with the probe beam and its HeNe beam. Then, once the 2.5-meter hot cell was filled, the experiment at hand could begin.

The experiments performed were of two classes: $R-R$ relaxation, the first, and $V-V$ relaxation, the second. Each class divided also into two natural parts. For the $R-R$ work these two were the common upper-level, or three-level, measurements and the four-level, or $\Delta \mathrm{J}$, measurements. The $V-V$ measurements consisted of, first, the hot-band work and, then, the sequence band work. Each measurement will now be described in the above order.

The three-level $R-R$ measurements began with a series to study the j-dependence of the pump line. Scope traces of the relaxation were taken for the pump/probe combinations $P(10)_{10} / P(10)_{9}, P(22)_{10} / P(22)_{9}$, and $P(34)_{10} / P(34)_{9}$. In each case signals were recorded at pressures of 1,2 , 3 , and 4 torr. The effect of reversing the pump and probe was then checked with $P(22)_{9} / P(22) 10^{\circ}$ The decay time of this signal and that of the $P(22) g$ probe, mentioned above, sinould have been the same if there was truly no dependence on the lower probe level. The scope trace at two torr, for this 10-um probe experiment, is shown in Fig. IV-5. 


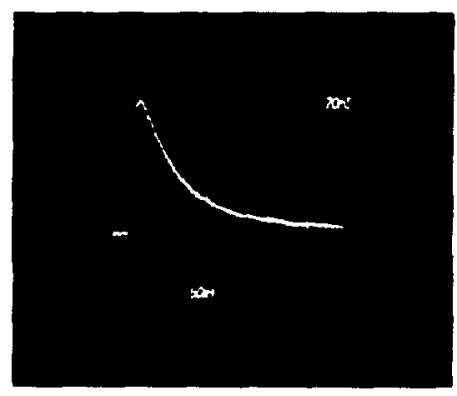

Figure IV-5. Observed Decay of the Pumped Level

Shown is the scope trace for the experiment with the pump/probe combination $P(22)_{g} / P(22)$, 0 at a pressure of 2 torr. Scales are $20 \mathrm{~ns}$ and $50 \mathrm{mV} / \mathrm{small} \mathrm{div.}$ 
At this point a study was also made to see that the fall times had no pump intensity dependence. Such a dependence could mean e::cessive leak-through at the shutter or perhaps a coherent propagation effect in the gas. No such dependence was observed, but it was decided that, for the analysis, decay rates should be measured only for the region of the signal greater than $8 \mathrm{~ns}$ after the rise. This is twice the width of the pump and would assure free decay for the population, allow for the possibility of pump signal in the detector, and still leave most of the signal for analysis.

A second type of three-level measurement was performed by utilizing the $R$ branch as a probe. Data were taken with $P(22)_{g} / R(22)_{g}$. This is essentially a common lower level $(020, \mathrm{~J}=22)$ configuration so is a measurement of relaxation of the lower pump level. The results should indicate if 001 and 020 relaxation are similar. This would have a bearing on the importance of the 000-VV rotational exchange mechanism which of course cannot play a part in the 020 process. The interpretation is somewhat complicated by the fact that the probe also samples an upper level $(001, J=23)$. However, the lower level is directly involved in the pumping and should dominate the signal behavior. The $10-\mu m$ analog, $P(22) / 10 / R(22) / 10$, was also checked. 
The second phase of the $R-R$ measurements consisted of four-level experiments to determine the character of the $\mathbf{j}$-dependence of rotational relaxation. Data were taken with laser configurations $P(24)_{9} / P(24+\Delta J)_{10}$. with $\Delta \mathrm{J}= \pm 2, \pm 4, \pm 6, \pm 10, \pm 14,-18$, a 11 at a pressure of 4 torr. Sample traces are shown in Fig. IV-6 for $\Delta J=$ $+2,+6,+10$. These show three features of interest: the risetime, the overshoot, and the flat rotational equflibrium region. Additional traces were taken at faster sweep rates, for each $\Delta J$, so the risetime of the response could be studied.

The $V-V$ measurements of $v_{3}$ transfer began with the hot-band probe studies. The purpose was basically to observe the $001+011$ process which can be compared with other work and which is expected to play an important role in the $001+002$ data. The first series of measurements was a pressure dependence study for $P(24)_{g} / P(22)_{1}$. The pressures were 5, 10, and 15 torr. A sample trace of the observed response at 10 torr is shown in Fig. IV-7. The signal rises in a smooth monotonic fashion to a temporary equilibrium value as expected. The dependence on the $011 \mathrm{~J}$ value was then examined to make sure there were no unexpected differences for different lines.

While continuing to look for the unexpected, the effect of using a 10-um pump was examined. An 

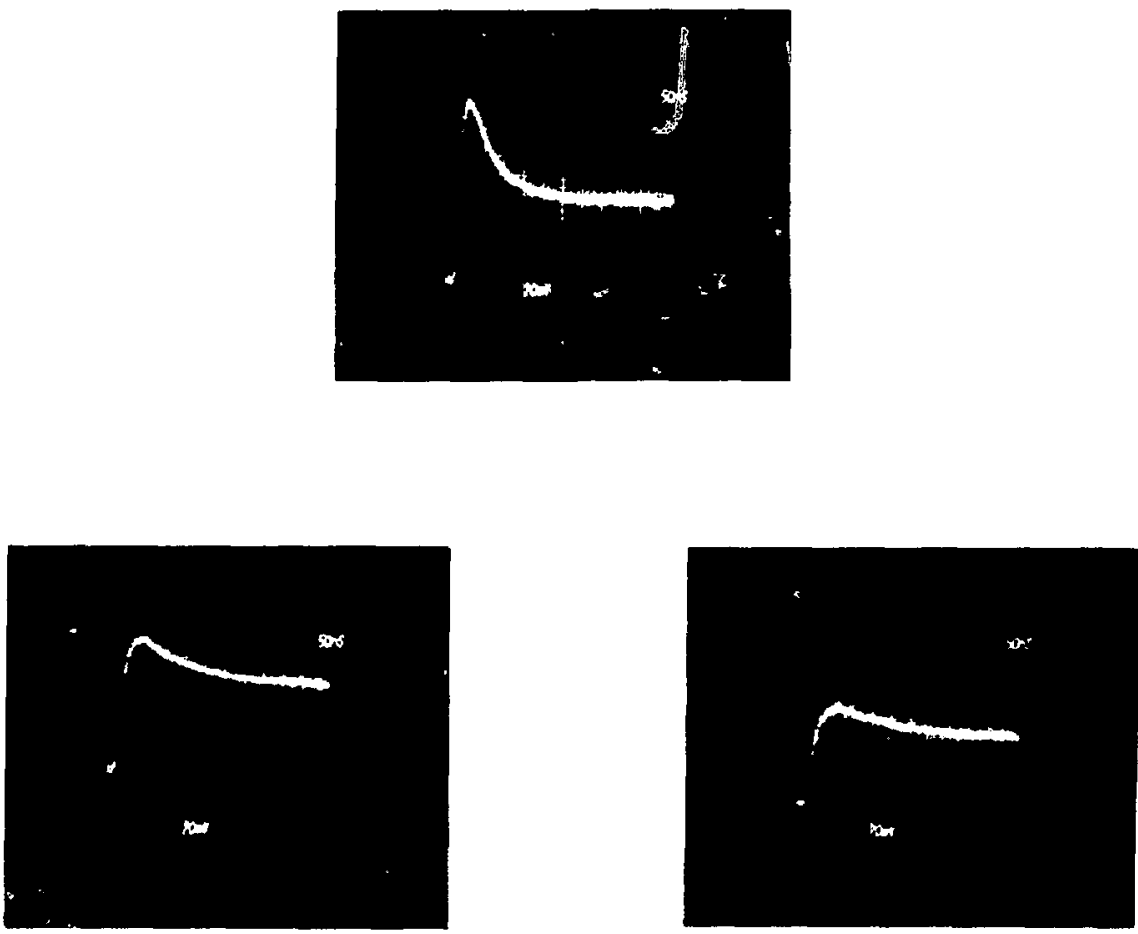

Figure IV-6. Samples of J-Dependent Relaxation

Rotational relaxation traces are shown for $\Delta \mathrm{J}=+2$, +6 , and +10 at 4 torr. The most interesting features are the fast risetimes, the overshoot, and the rotational equilibrium. Scales are $50 \mathrm{~ns}$ and $20 \mathrm{mV} / \mathrm{small} \mathrm{div}$. 


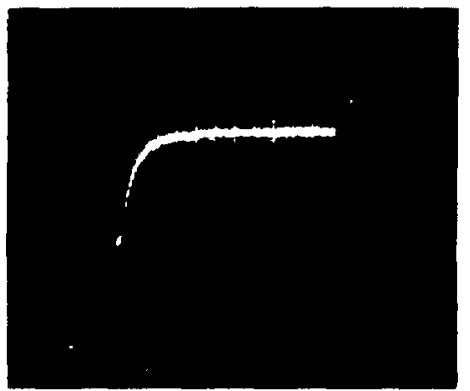

Figure IV-7. Hot-Band Probe Data

This signal shows the $n_{0} 1$ response in the $P(24)_{9} / P(22){ }_{1}$ experiment at 10 torr. The monotonic rise to equilibrium is the expected behavior. Scales are $100 \mathrm{~ns}$ and $20 \mathrm{mV} / \mathrm{small}$ div. 
unexpected and very interesting difference was, indeed, observed between the signals produced by $9-\mu m$ and 10-um pumps. Samples of these data at 10 torr are shown in Fig. IV-8. The rise in the 10-um pump case is seen to overshoot the equilibrium value and then fall back to it. An additional fast process must be occurring for this pump and probe combination.

The second phase of the $v_{3} V-V$ experiment was the sequence-band probe study. Since the $001+002$ rate constant was expected to be very sensitive to the bump feature discussed in Section III.B.4. and Fig. III-6, an effort was made to try to observe it. An initial attempt, with $P(24)_{g} / P(23)_{10}$, showed some evidence of the bump, but the signal to noise ratio, $S / N$, was definitely less than one.

In order to increase the $S / N$ ratio, several steps were taken. Straightening the sagging probe tube increased its power by a much needed factor of five. The probe beam overlap with the pump, through the hot cell, was also improved. In addition the optics in the screen room were adjusted to maximize the grating transmission. The signal amplification was also increased by a factor of two. In the bias circuit, the 50- $\Omega$ splitter was removed for a factor-of-two increase in the signal, and the bias was raised from $-180 \vee$ to $-300 \mathrm{~V}$. The risk of reflections and bias oscillations did not seem important at this point. 


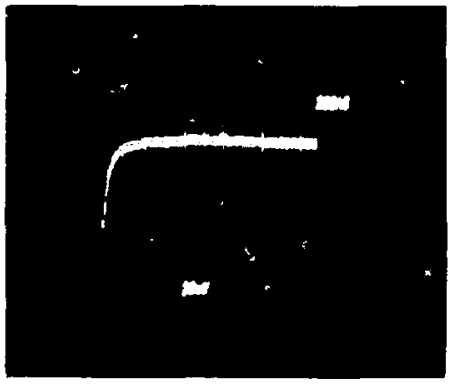

(a)

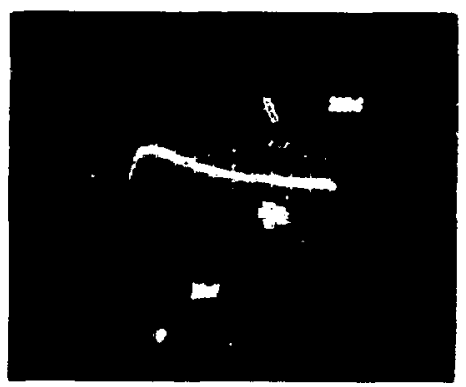

(b)

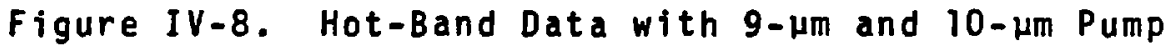
The difference in the observed signal when using

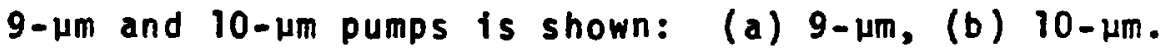
The overshoot in the $10-\mu m$ case indicates an additional fast process is involved. The pressure is 10 torr. Scales are $200 \mathrm{~ns}$ and $20 \mathrm{mV} / \mathrm{small} \mathrm{div}$. 
Convincing, reproducible data were finally obtained at 10 and 20 torr for $P(20)_{9} / P(19)_{10}$. A sample of the 10 torr results is shown in $F$ ig. IV -9 . The first $40 \mathrm{~ns}$ is just baseline ( $5 \mathrm{~ns} / \mathrm{small}$ box). At $10 \mathrm{~ns}$ before the center line, the response begins. This start time was precisely measured by opening the grating aperture and allowing some of the 4-ns pump into the detector. After this time point, the bump feature can clearly be seen, and then, the zero crossing, and finally, the strong negative increase.

A slower sweep speed was then used to easily obtain the $001+101$ relaxation traces at 5, 10, and 20 torr. The 10-torr trace is shown in Fig. IV-10.

This concluded the laboratory portion of the experimental program.

The final step in the data acquisition process was the digitization of the scope traces for later analysis. This was accomplished with a Tektronix 3954 Digitizer Tablet and the same Eclipse minicomputer used for the $V-V$ mode? studies. The digitizer system utilized an opaque projector to create an enlarged image on the tablet surface. The digitization was accomplished in two steps. First, a box of four-grid points, outside the trace, was digitized to provide full scale conversion factors and also to check for distortion and rotation in the 


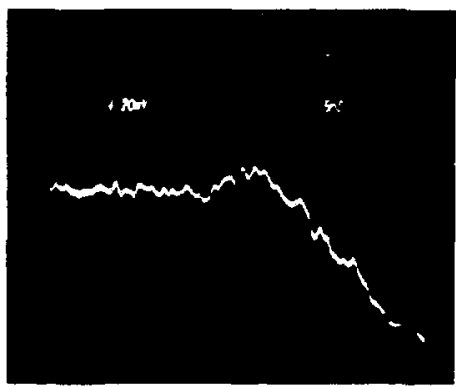

Figure IV-9. Fast Sweep Sequence-Band Data at 10 Torr The signal for $P(20)_{9} / P(19)_{10}$ with scales of 5 ns and $20 \mathrm{mV} / \mathrm{small}$ div. is shown. The bump and subsequent zero crossing can be seen after the $40 \mathrm{~ns}$ of iritial baseline. 


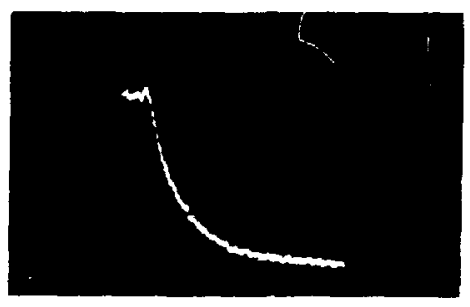

Figure IV-10. Sequence-Band Data with Slower Sweep

The trace shows the longer-term behavior of $n_{002}-$ ${ }^{n} 101$ at 10 torr. The initial baseline and bump feature are barely discernible. Scales are $50 \mathrm{~ns}$ and $50 \mathrm{mV} / \mathrm{small}$ div. 
projection. The second step was simply to digitize 50 to 100 points from the projected scope trace. The points, from each trace, were stored in a file with a file name constructed from the photo date and number. These files could be easily accessed by the analysis programs. 
CHAPTER $V$

\section{ANALYSIS AND RESULTS}

In this chapter the results of the investigation are given. In the preceding chapter the procedures were described by which the raw data were obtained. Extraction of rate coefficients from these data involves some mode? fitting. The details of these procedures are also described here. In all cases the fitting was accomplished by programs utilizing the method of least-squares minimization for arbitrary fitting functions (Bevington, 69, p. 204). The results of the rotation transfer experiments are discussed first and then the results for the vibration transfer.

A. Rotational Energy Transfer Results

The two categories of rotational relaxation experiments were the pumped-level decay measurements and the neighboring-level response measurements. From the former, effective rotational relaxation rate constants have been derived for the various pump/probe combinations. The data from the latter have been converted to population response plots similar to those which were useful in the model 
studies (Chapter III). The 10-90\% risetime for each $\Delta J$ was also determined.

1. Pumped-Level Relaxation

This experiment consists of a series of three-level double-resonance measurements with a common upper level. In each case an 001 rotational level was subjected to an overpopulating perturbation by the pump laser. The subsequent return to equilibrium, of the same level, was monitored by the probe transmission. This is a direct observation of the relaxation of the individual rotational level. A sample of the data for the level $(001, J=21)$ with pump/probe combination $P(22)_{9} / P(22)_{10}$ is shown in Fig. IV-5. The other data in the series were for $P(10)_{10} / P(10)_{9}, P(22)_{10} / P(22)_{9}, P(34)_{10} / P(34)_{9}$, $P(22)_{9} / R(22)_{g}$, and $P(22)_{10} / R(22)_{10}$.

The signal decays observed were analyzed with the fitting function described by Eq. II-40 and Eq. II-43. The time dependence of $n(t)$ was assumed to be an exponential decay from some initial pumped population to a final equilibrium. The initial and final amplitudes and $\tau_{r}$ were the three variables of the fit. The region of the trace fitted was trat portion between 8-10 ns after the peak and the end of the trace. The procedure was repeated at each of the four pressures studied, and the 
resulting values of $\tau^{-1}$ were plotted vs pressure in the manner shown in Fig. $V-1$ for $P(22)_{9} / P(22)_{10}$. The expected linear pressure dependence is evident.

The resulting four data points were subjected to a fit by a straight line through the origin. The one parameter allowed to vary was the slope. The fitted line is also shown in the figure. The slope gives the desired value of $k_{r}$. The statistical errors in the fit can be used to derive an expression for the standard deviation in the slope measurement (Bevington, 69, p. 114).

The data from each pump/probe experiment were analyzed by the above steps. The results are given in Table $V-l$ and are discussed in the following chapter.

2. J-Dependence of the Relaxation

This experiment consisted of four-level double resonance measurements in which one 001 rotational level was pumped and neighboring 001 rotational levels were probed to observe their response to the perturbation. The population plots from the model studies in Chapter III showed this type of experiment is sensitive to details of the collision mechanism. The difference between $\mathrm{J}$ for the probed level and the pumped level defines $\Delta J$. Samples of data are shown in Fig. IV-6. The experiment resulted in data for $\Delta J= \pm 2, \pm 4, \pm 6, \pm 10, \pm 14,-18$. 


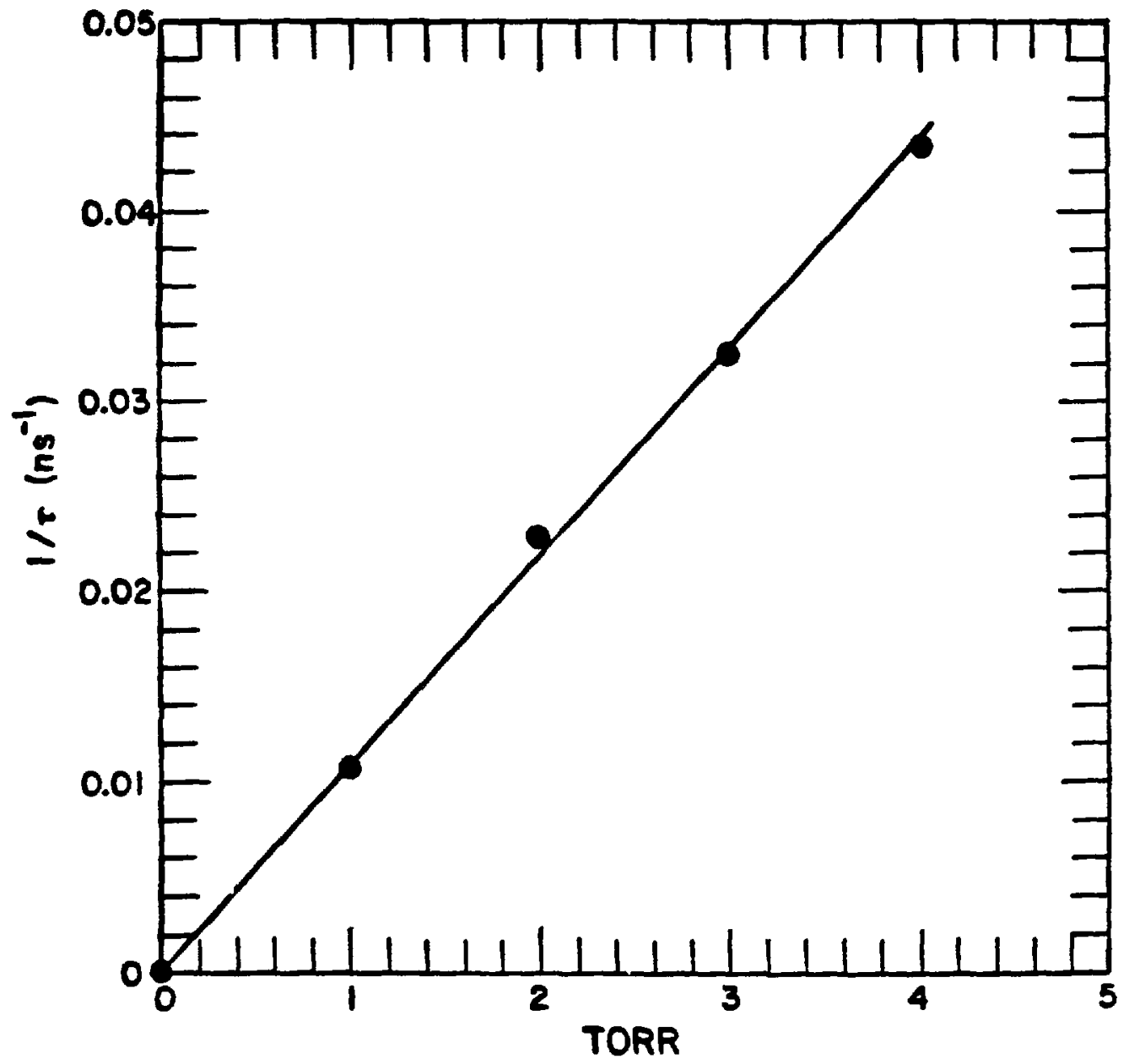

Figure V-1. Sample Plot of $:^{-1}$ vs Pressure

The daca points and the straight line fit through the orlgin are shown for $P(22)_{9} / P(22){ }_{10}$. The slope of the ifne determines the rotational relaxation rate coefficient. 
TABLE $V-1$

PUMP / PROBE

1) $P(10)_{10} / P(10)_{9}$

2) $P(22)_{10} / P(22)_{9}$

3) $\quad P(34)_{10} / P(34)_{9}$

4) $\quad P(22)_{9} / P(22)_{10}$

5) $P(22)_{9} / R(22)_{9}$

6) $P(22)_{10} / R(22)_{10}$

$$
k_{r}\left(\times 10^{7} \sec ^{-1} \operatorname{torr}^{-1}\right)
$$

$1.08 \pm .04$

$1.10 \pm .03$

$1.19 \pm .05$

$1.10 \pm .02$

$0.97 \pm .08$

$0.98 \pm .10$ 
The objectives of the analysis of the $\Delta J$ relaxation data were to obtain population response plots, as in Chapter III, and to extract risetimes. No rate constants were determined.

The data consists of two traces for each nearby level at 4 torr. One shows the long-term relaxation, and the other shows the short-term response. The first shows the response all the way to rotational equilibrium. The second shows, very clearly, the initial rise of the trace and extends through the overshoot region of the response.

To plot the various $\Delta J$ signals on the same graph, as population plots, requires an amplitude scaling of the data from each photo. This normalization was performed in the following way. The change in the pumped level density was assumed to reach an, approximately correct, equilibrium value of $10^{13} \mathrm{~cm}^{-3}$. The corresponding equilibrium populations for the other 001 levels of interest were then calculated. Each of the long-term traces was then normalized so it would have that value at a specific elapsed time well into the equilibrium regime. The resulting population plots are shown in Fig. V-2 and Fig. $V-3$ for $\Delta J>0$ and $\Delta J<0$, respectively. The results are clearly not independent of $J$. The overshoot for $\Delta \mathrm{J}= \pm 2$ is $100 \%$ or more. 


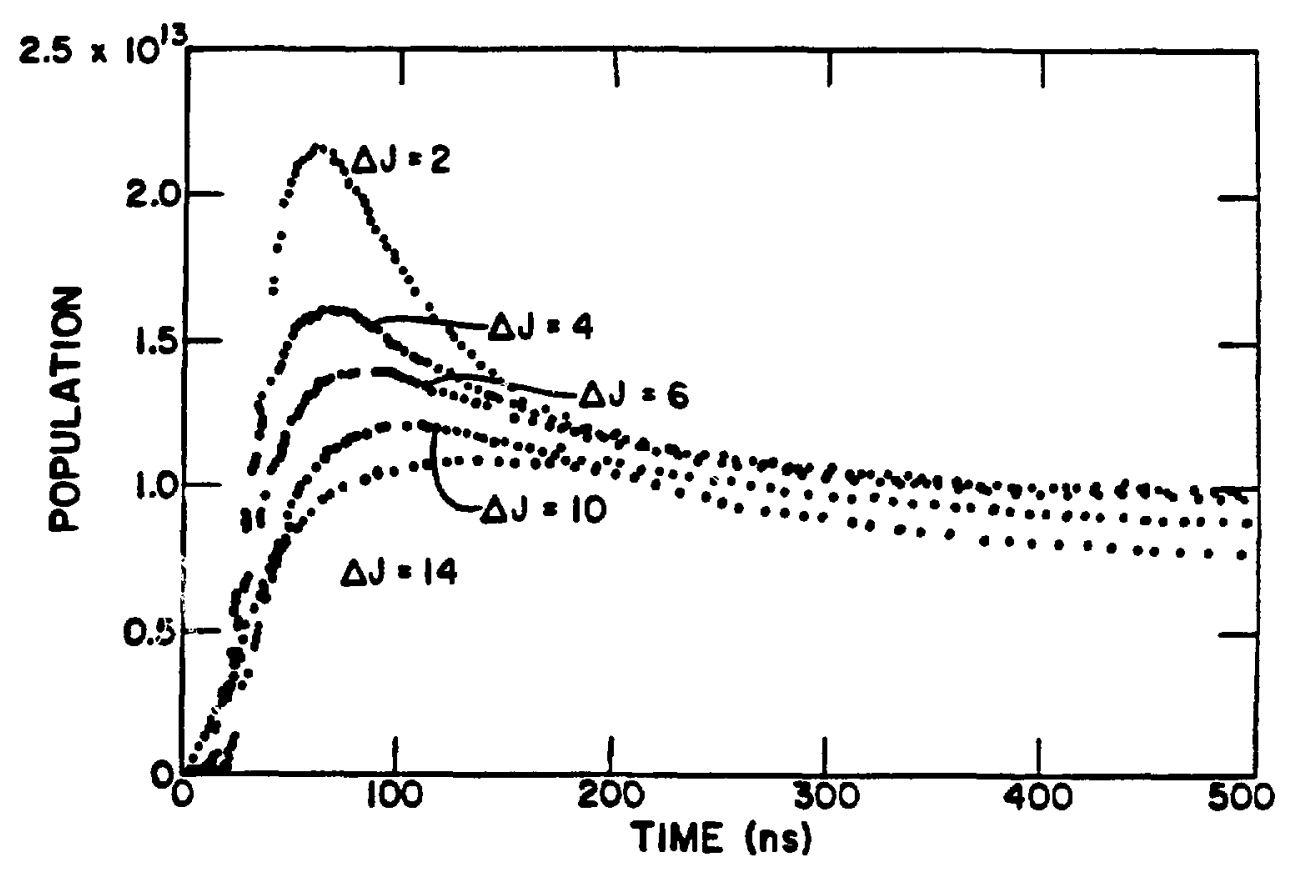

Figure V-2. Long-Term J-Dependence of Rotational Relaxation

Shown are plots of the response of nefghboring $j-1$ evels at 4 torr derived from the $P(24)_{g} / P(24+\Delta J)_{10}$ data with $\Delta J>0$. 


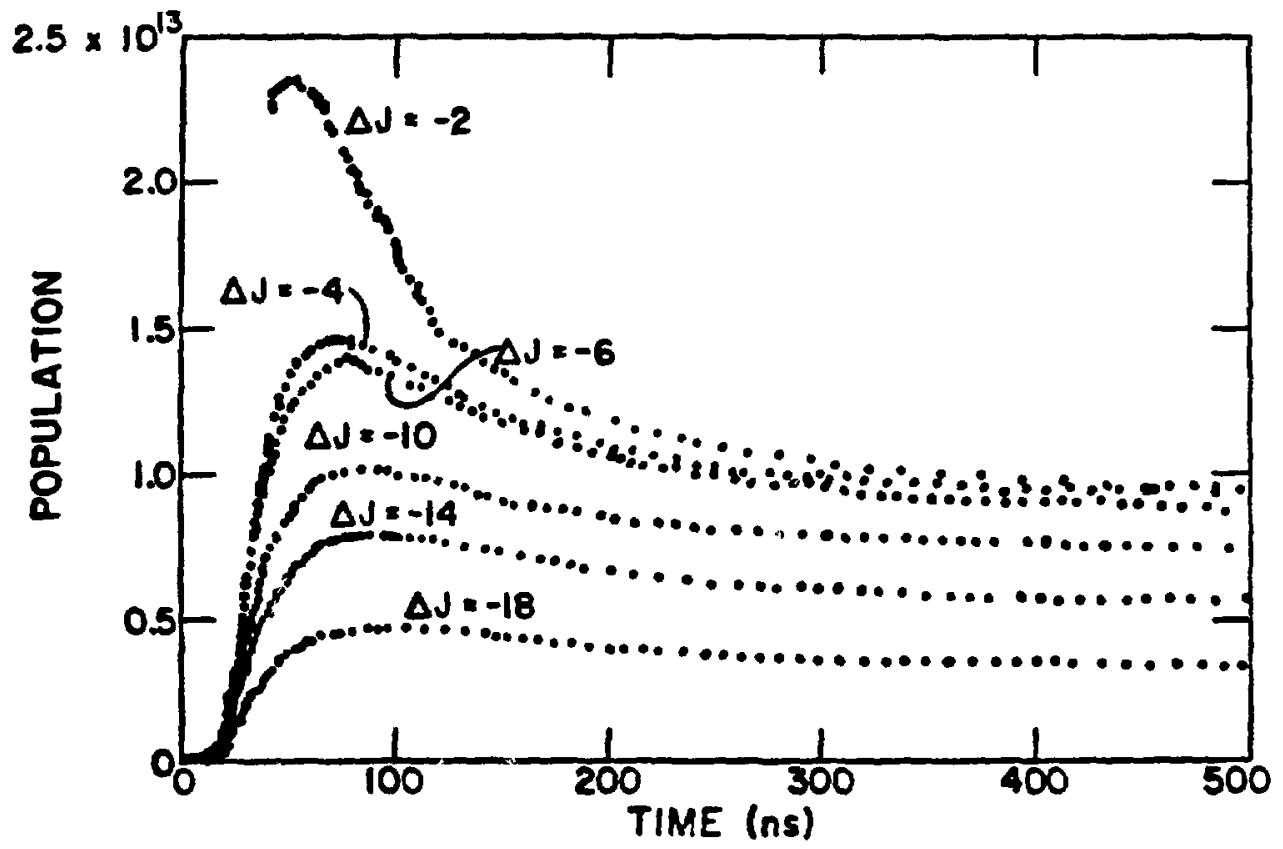

Figure V-3. Long-term J-Dependence of Rotational Relaxation.

Shown are plots of the response of neighboring $j-l e v e l s$ at 4 torr derived from the $P(24)_{9} / P(24+\Delta J)_{10}$ data with $\Delta J<0$. 
The normalization for the short term signals is more difficult since the traces do not reach the equilibrium regime. This problem was overcome by equating the peaks of the short term data to the peak populations obtained from the long-term responses. The resulting calculated populations are given in Fig. $V-4$ for $\Delta J>0$ and in Fig. $V-5$ for $\Delta \mathrm{J}<0$.

Both the long term and short term plots show an anomaly for the $\Delta \mathrm{J}=-4$ and -6 cases. A likely explanation is that, for the $\Delta J=-4$ measurement, the probe was accidentally adjusted to $\Delta j=-6$. If this were : case, two $\Delta J=-6$ traces would result with a slight difference in normalization, as sean in Fig. $V-5$.

The 10-90\% risetimes were easily obtained from the short term data. The plotted results for $\Delta \mathrm{J}>0$ and $\Delta J<0$ are shown in $F i g . V-6$. The $\Delta J= \pm 2$ values are very nearly equal. The $\Delta J=-4$ value has been purposefully omitted.

The results are further discussed in the next chapter.

B. Rapid Vibrational Energy Transfer

The main thrust of the vibrational transfer study was the determination of $k_{002}$. This has been accomplished a long with the the dietermination of $k_{101}$ and $k_{01}$. The techniques for orriving at these results are discussed 


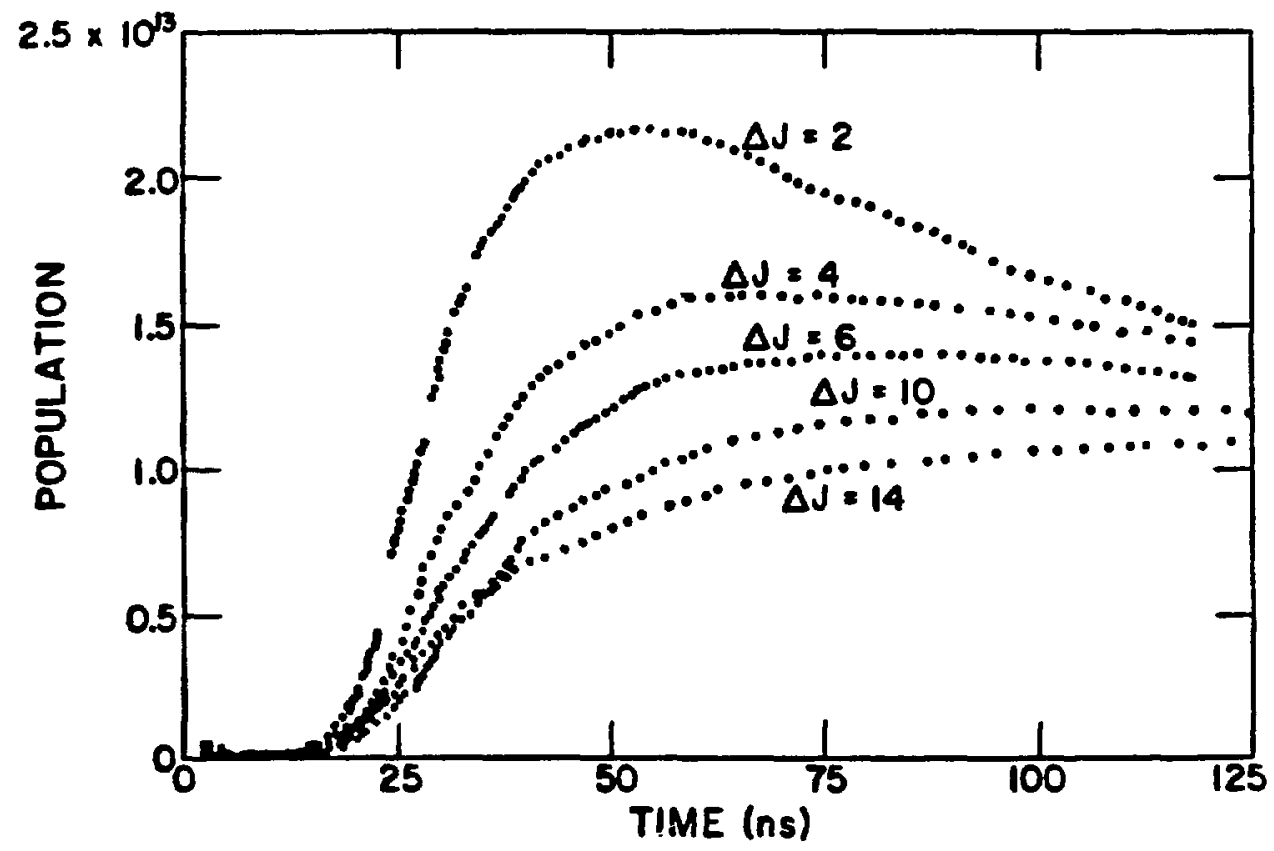

Figure V-4. Short-Term J-Dependence of Rotational Relaxation

Shown are plots of the response of neighboring $j-l e v e l s$ at 4 tor derived from the $P(24)_{g} / P(24+\Delta J)_{10}$ data with $\Delta J>0$. 


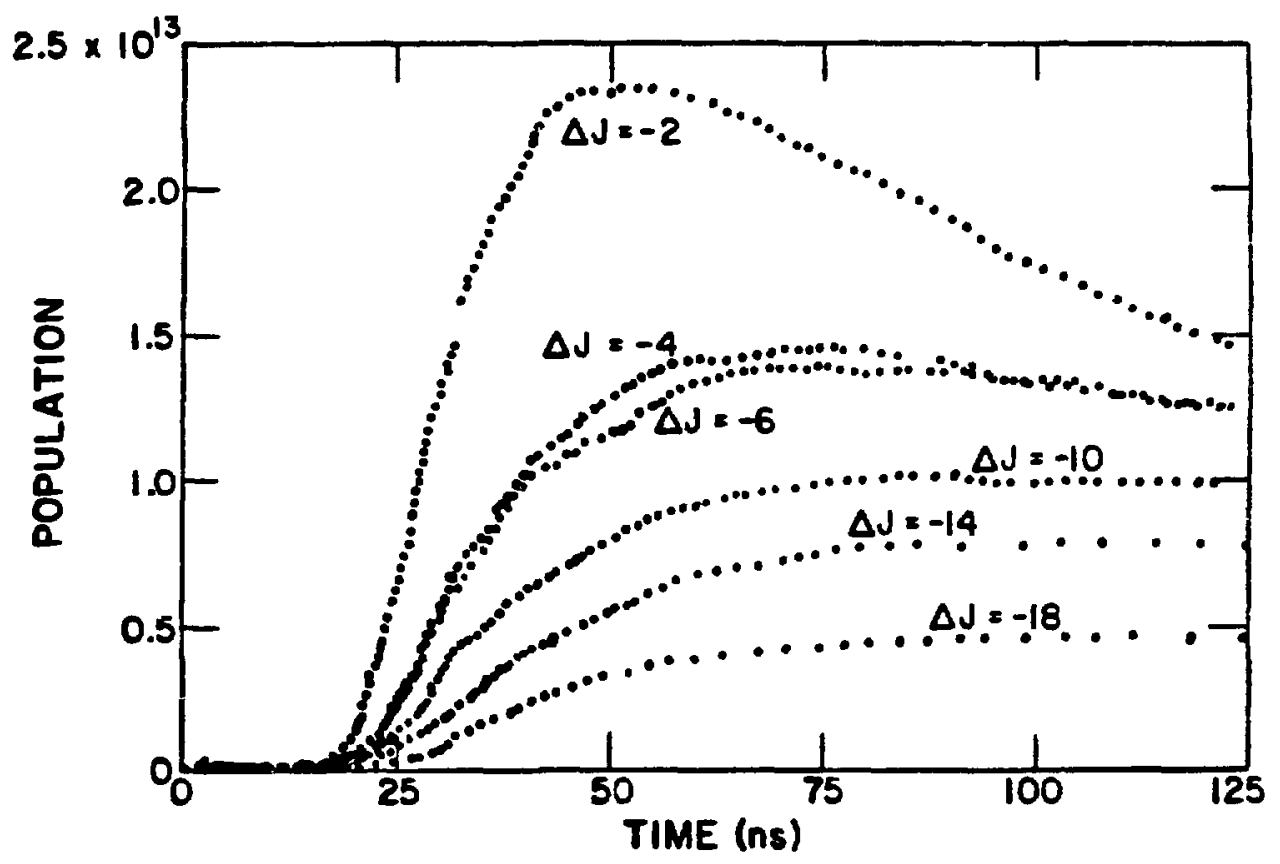

Figure V-5. Short-Term J-Dependence of Rotational Relaxation

Shown are plots of the response of neighboring $j-1 e v e 1 s$ at 4 torr derived from the $P(24)_{g} / P(24+\Delta J){ }_{10}$ data with $\Delta \mathrm{J}<0$. 


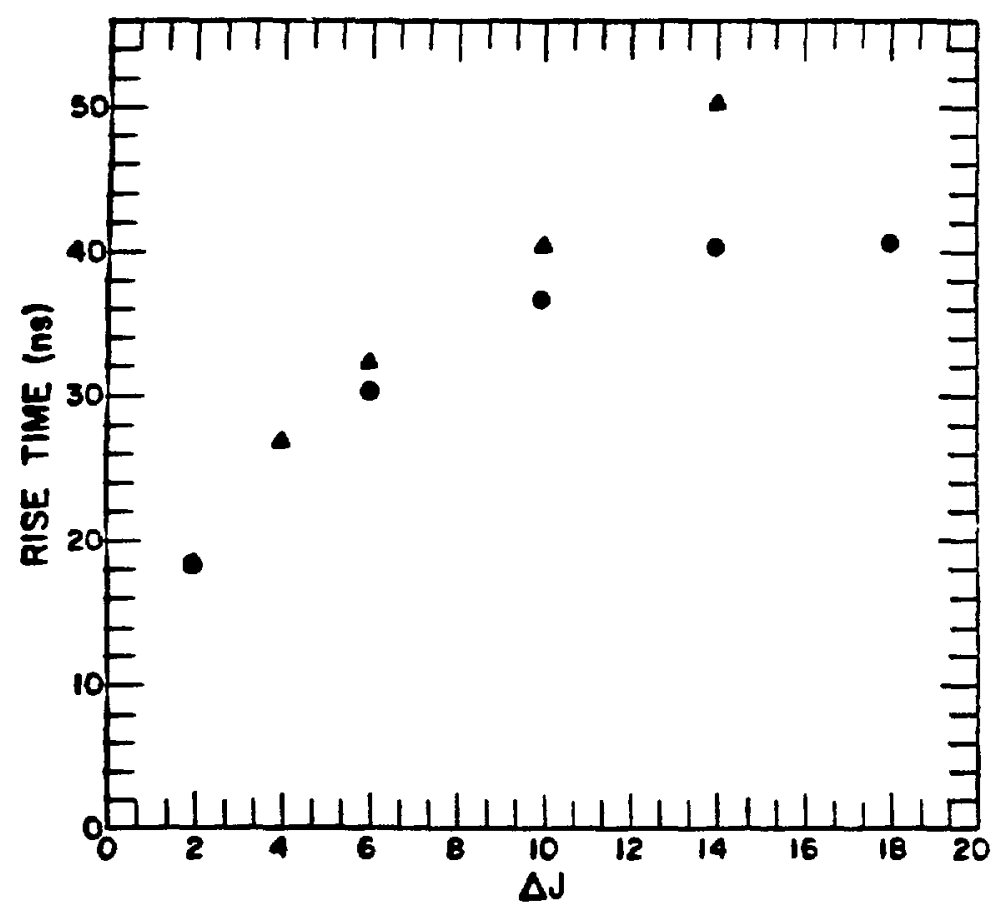

Figure $V-6$. Risetimes of the $\Delta J$ Responses

The 10-90\% risetimes are shown for the $\Delta J$ levels at 4 torr. Circles $+\Delta J<0$; triangles $+\Delta J>0$. The $\Delta J= \pm 2$ values are nearly equal. There is no $\Delta \mathrm{J}=-4$ point. 
below. In addition, some of the hot-band $(011+110)$ probe data has been interpreted as evidence for resonant dipole-dipole transfer of a $v_{2}$ quantum. This interpretation is also described.

1. The $\nu_{3}$-Transfer Results

The reactions and associated rate constants studied in this $700^{\circ} \mathrm{K}$ experiment are
a) $001+010 \leftrightarrow 011+000 ; k_{011}$
b) $001+100 \leftrightarrow 101+000 ; k_{101}$
c) $001+001 \leftrightarrow 002+000 ; k_{002}$

The investigation utilized hot-band $(011+110)$ and sequence-band $(002-1.01)$ probe lasers. The relevant experimental parameters in the rate constant measurements are summarized below.

$\begin{array}{lll}\text { Constant } & \frac{\text { Pump/Probe }}{k_{011}} & \frac{P(\text { Torr })}{k_{101}} \\ & P(24)_{g} / P(22)_{11} & 5,10,15 \\ & P(20)_{g} / P(19)_{10} & 5,10 \\ k_{002} & (\text { s low }) & \\ & P(20)_{9} / P(19)_{10} & 10,20 \\ & \text { (fast) }\end{array}$

In the second column, the relative sweep speed of the scope trace is indicated. The weak signal in the 
fast-sweep sequence-band data and the importance of the bump feature for determination of $k_{002}$ have been discussed, extensively, in the preceding chapters (II, III, and (V).

The analytic expressions for $n_{002}, n_{101}$, and $n_{0} 1$ given by Eqs. III-35, III-28, and III-26, respectively, have been utilized as fitting functions to extract values of $k_{002}, k_{101}$, and $k_{011}$ from the sequenceband and hot-band probe data. Initially it was not clear that this could be accomplished, in a convincing way, since there was concern about the quality of the bumpfeature data and about the validity of the ${ }^{n} 101$ model. In least-squares fitting, it is always a good idea to minimize the number of fitting parameters. The procedure that was developed for fitting was one in which as few parameters as possible were allowed for each step. In this process the 10 torr data from the hot-band probe, fo: the fast-sweep sequence band, and for the slow-sweep sequence band were used. The variables required are, in addition to the three rate constants, the amplitude of each trace and a time, $t_{0}$, indicating where, on each trace, the signal begins. It was decided that fitting each trace individually, allowing only the most sensitive 
$k$ to vary, was the wisest approach. The two other traces could then, successively, be fitted in the same manner, each with only one rate constant varying. The bumpfeature data, of course, did not need a $t_{0}$ parameter as its value had been measured by letting some pump signal through to the probe detector. For each successive trace the current fitted values of the other two rate constants were used in the fitting function. This three step process was then iterated until convergence resulted, i.e., until the parameters were no longer changing significantly.

A computer study, including the digitization of numerically generated data traces, was made to test the capabilities of the method. The rate coefficients used in the data generation were reproduced to about $5 \%$. This is an indication of the best accuracy that could be expected.

When this procedure was applied to the actual 10 torr data, convergence was found to occur with three iterations, for the chosen starting values. The results are given in Table $V-2$. The digitized cata and the final-fit curves are shown in Figs. $V-7, V-8$ and $V-9$. The first is for the hot-band probe case. The second and third are the slow and fast sweeps of the sequence-band data, respectively. 


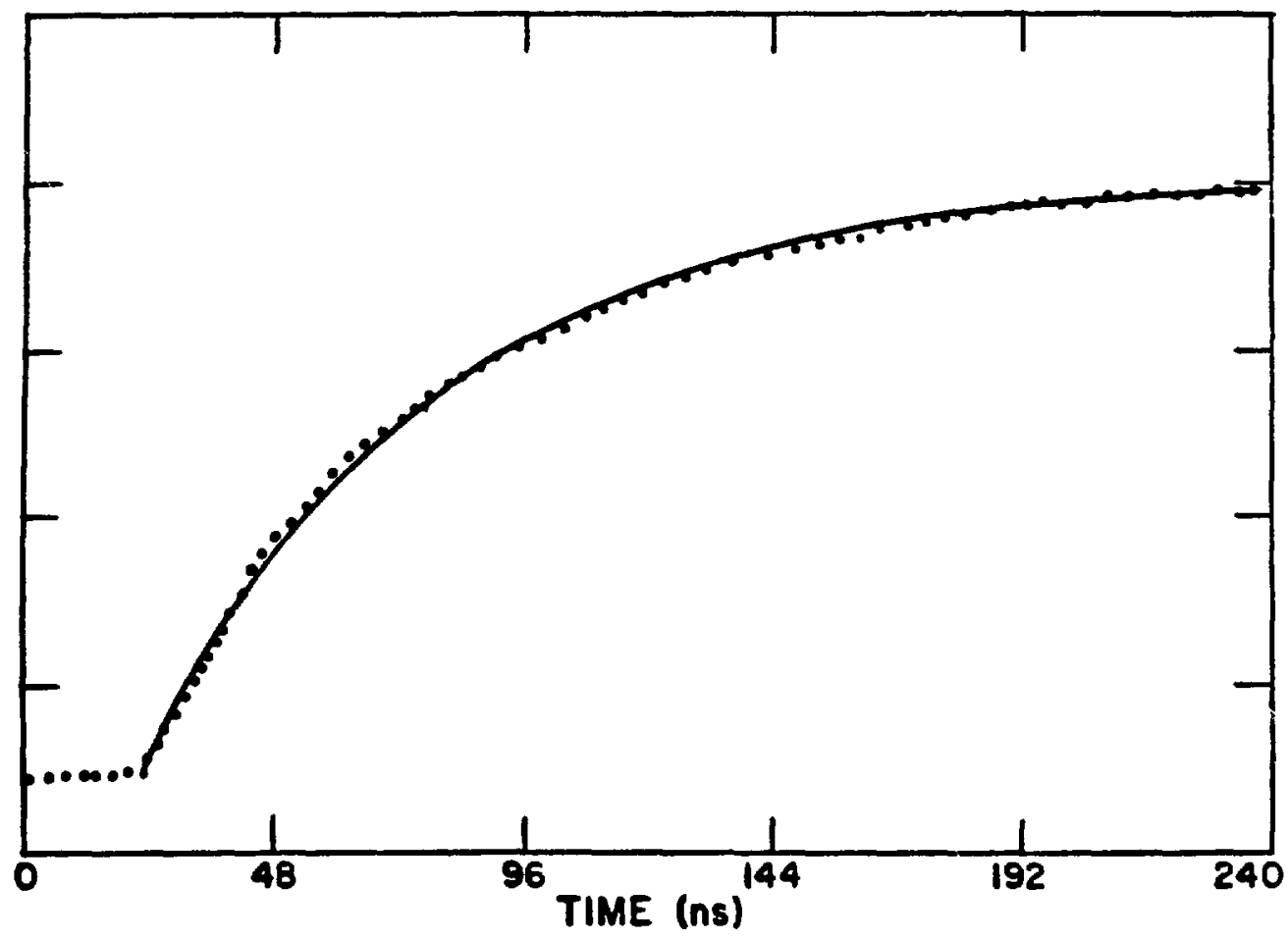

Figure V-7. Hot-Band Data and Fit

The digitzed hot-band probe signal is shown for the 10 torr case. Also shown is the final fitted solution from the three-level model. 


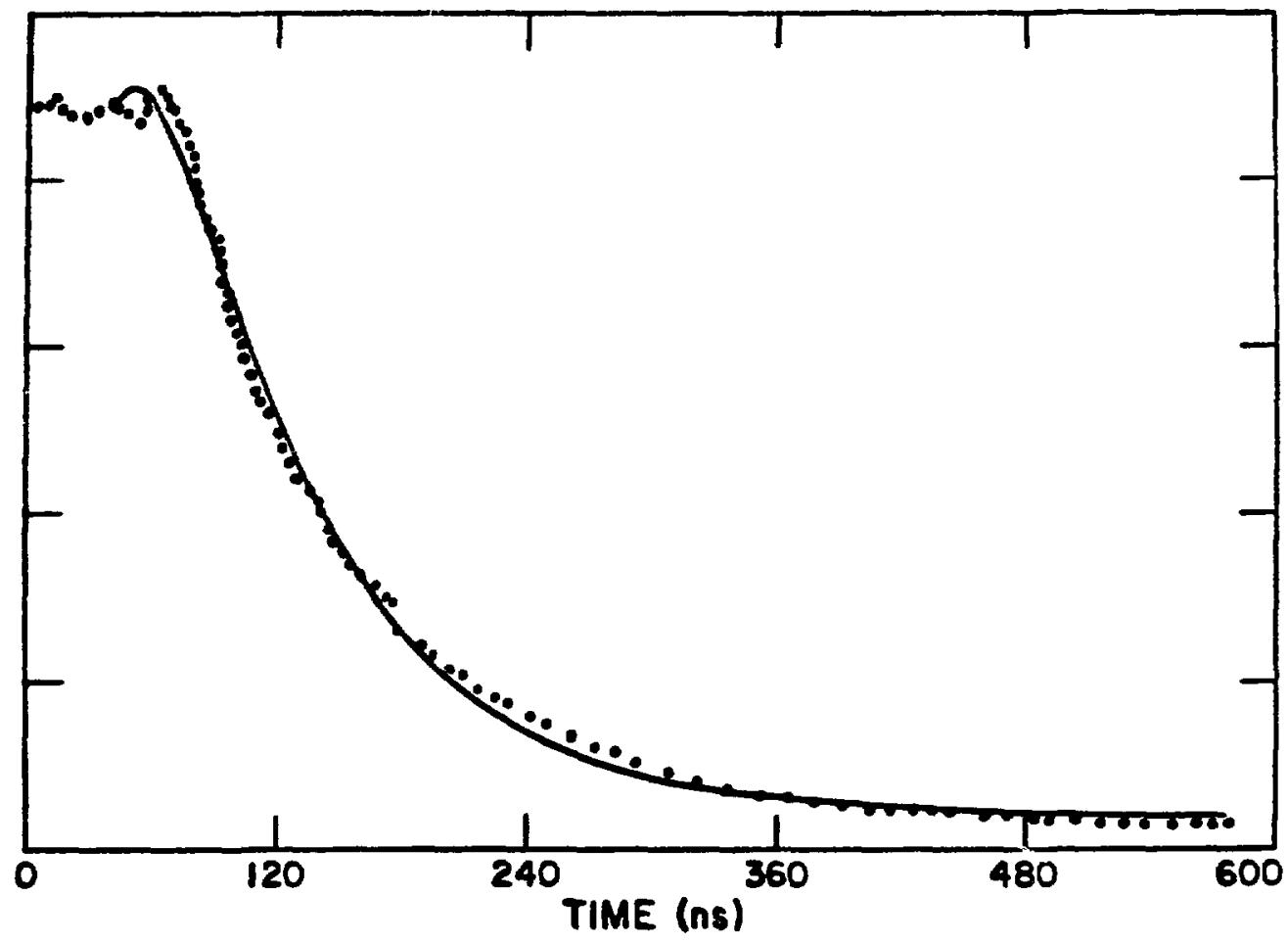

Figure V-8. Slow-Sweep Sequence-Band Data and Fit The digitized sequence-band probe signal is shown for the 10 torr case. Also shown is the final fitted solution from the combination of the three-level model and the $n_{002}$ solution. 


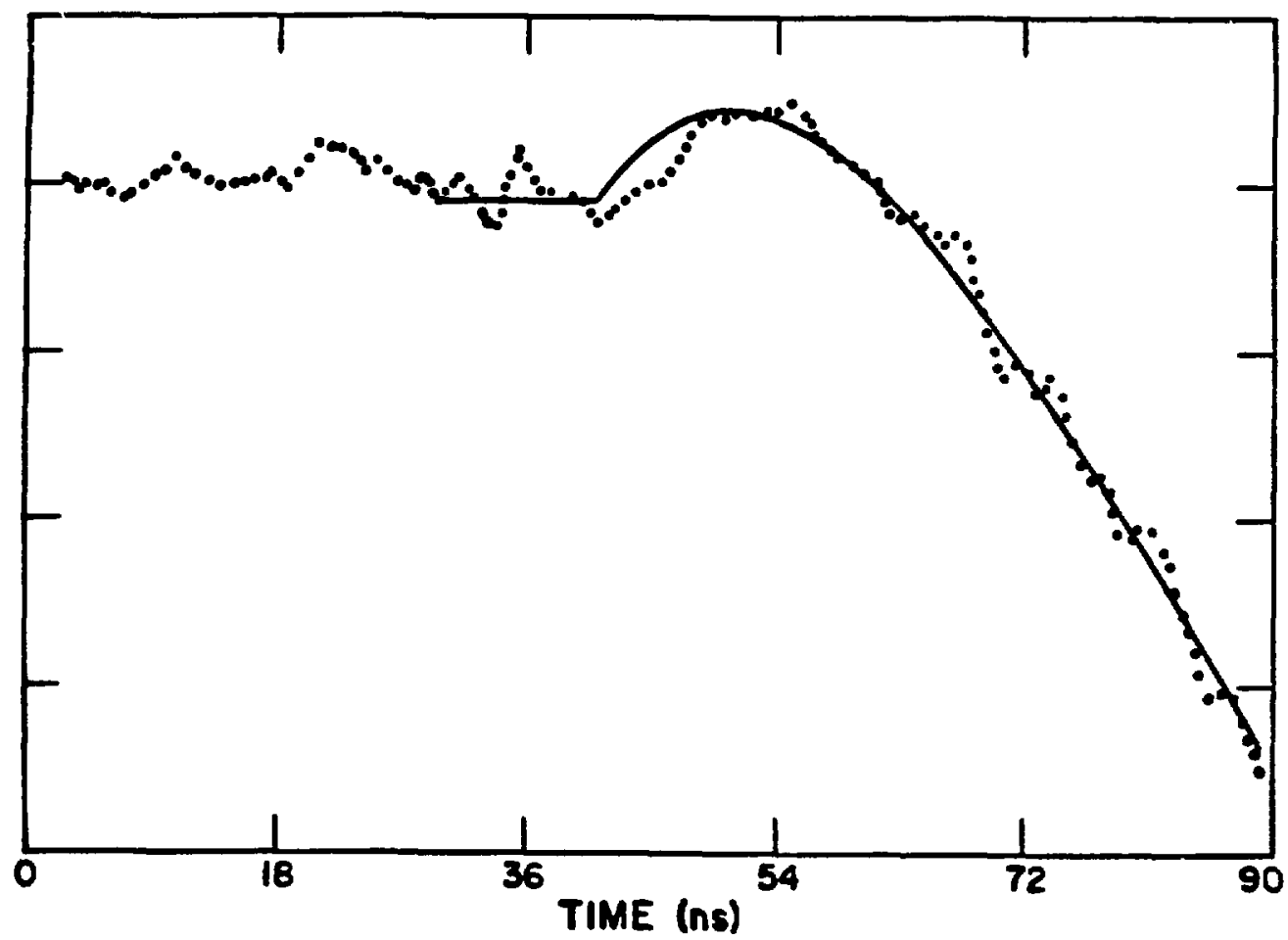

Figure V-9. Fast-Sweep Sequence-Band Data and Fit The digitized data for the bump feature of the sequence-band measurement at 10 torr is shown. The final-fit curve is also shown. The good reproduction of the zero crossing time is evident. 
In the least-squares fitting (Bevington, 69, $p$. 187) the function of merit which is minimized is called $x^{2}$ (chi-squared). It is a measure of the ratio of the deviations of the data from the fitted curve to the noise fluctuations in the data. A value of $x^{2}=1$ indicates the fit is as good as the data.

The noise must be estimated from the data traces and input to the fitting code. The relevant data are exemplified in the photos of Fig. IV-7, Fig. IV-9, and Fig. IV-10 for the hot-band probe, the fast-sweep sequence-band probe, and the slow-sweep sequence-band probe, respectively. The rms noise estimates were, in the same order, $3.0 \mathrm{mV}, 5.0 \mathrm{mV}$, and $7.0 \mathrm{mV}$. The values were obtained by taking $1 / 3$ of the peak-to-peak noise in analogy with the rms value of a sine curve.

The $x^{2}=0.2$ for $k_{011}(T a b l e v-2)$ indicates an overestimation of the noise, but, nevertheless, a very reasonable fit. The good quality of the fit $i$ also evident in the corresponding figure, $v-7$. On the other hand there appears to be a problem with $x^{2}$ in the k 101 case. The $x^{2}=1.7$ value is 1 arge. This may reflect a breakdown in the simple model and thus indicate that the fitting function is somewhat inappropriate. The figure, $V-8$, does not look that bad, however, except in the bump region. For $k_{002}, x^{2}=0.7$ is also reasonable. The 
third figure, $V-9$, shows the crossover point in the data is well reproduced. This is a very important feature and should indicate a reliable result for $k_{002}$.

A final concern, in the fitting, was whether or not the procedure led to unique results, independent of the starting values of the fitted parameters. This was tested by refitting with the initial values of $k_{002}$ and $k_{101}$ changed to $3.1 \times 10^{6}$ and $1.0 \times 10^{6}$, respectively. Convergence to the same final values occurrred, again in only three iterations. This was interpreted as evidence that, in each of the three cases, the function of merit was a smooth function of the parameters with a single minimum.

The rest of the $v_{3} V-V$ data consisted of two hot-band traces at 5 and 15 torr, slow-sweep sequence-band traces at 5 and 20 torr, and five fast-sweep sequence-band traces (three at 10 torr and two at 20 torr). Each of these was processed by fitting the appropriate single rate constant with the other two held fixed at the values obtained above. Thus there are three determinations, each, of $k_{011}$ and $k_{101}$, and six of $k_{002}$. These were used to estimate the statistical error for each coefficient. 
TABLE $V-2$
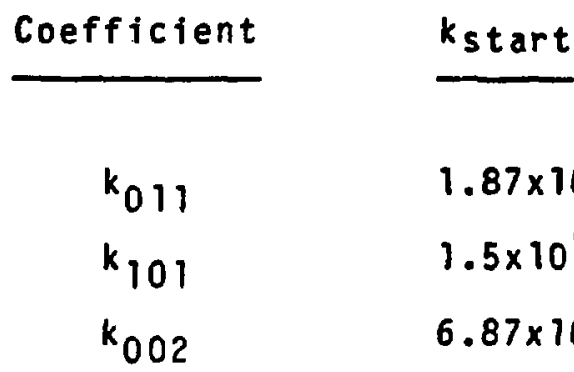

$k f i t$

$x^{2}$

$1.87 \times 10^{6}$

$1.95 \times 10^{6}$

0.2

1. $5 \times 10^{6}$

$1.54 \times 10^{6}$

1.7

$6.87 \times 10^{6}$

$7.62 \times 10^{6}$

0.7 
The final results were

$$
\begin{aligned}
& k_{011}=1.85 \pm .11 \times 10^{6} \mathrm{sec}^{-1} \operatorname{torr}^{-1} \quad v-1 \\
& k_{101}=1.60 \pm .22 \times 10^{6} \mathrm{sec}^{-1} \text { torr }^{-1} \quad v-2 \\
& k_{002}=7.61 \pm .24 \times 10^{6} \mathrm{sec}^{-1} \operatorname{torr}^{-1} \quad v-3
\end{aligned}
$$

In addition to statistical error, the $k_{002}$ error reflects a large contribution from an estimated $\pm 8^{\circ} \mathrm{K}$ temperature uncertainty. Model studies had shown the crossover time to be very sensitive to temperature. This surprising sensitivity was traced to the strong temperature depentence of the equilibrium 001 population which plays an important role in the 001+001 $\rightarrow 002+000$ collision process. The resulting $k_{002}$ error is stilla rather small $\pm 3 \%$. As discussed above, the computer simulation of the data fitting procedure could only reproduce the input $k-v a l u e s$ to $\pm 5 \%$. Thus the quoted $k_{002}$ error is, admittedly, probably too small.

The above results will be discussed further in the next chapter.

2. The $v_{2}$-Transfer Hypothes is

In the hot-band probe $(110+011)$ studies of $v_{3}$ transfer, the pump was normally the 9-um transition 
$(001+020)$. As a check that the lower probe level was having no effect on the signal, the signal was examined with the 10-um pump $(001+100)$. An unexpected difference,

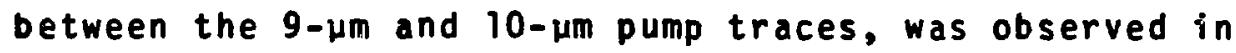
the hot-band transmission. Samples of the 10-torr data are shown in Fig. IV-8. The overshoot for the 10-um pump is clearly different from the 9-4m case. A study of pressure dependence, pump/probe line dependence, and laser-intensity dependence was consistent with an interpretation that an additional fast kinetic process was responsible.

The different behavior observed for 9-4m and

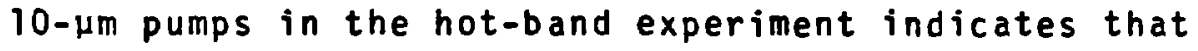
population is drained from the 110 level when the 100 level is depleted. A likely cause is the resonant, $v_{2}$ transfer, dipole-dipole reaction

$$
\left(10^{0} 0\right)+\left(01^{1} 0\right) \leftarrow\left(11^{1} 0\right)+\left(00^{0} 0\right)
$$

with $\Delta E_{v}=-22 \mathrm{~cm}^{-1}$. This is an especially interesting possibility because each molecule is required to undergo a $\Delta \ell= \pm 1$ transition. This means strong $Q$ transitions would be allowed unlike any of the $v_{3}$ reactions studied. These transitions are discussed in Section II.A. 
Assuming this reaction is responsible for the observations, the rate equation governing the process is

$$
\dot{n}_{110}=-n_{110} N_{0} k_{110}+n_{100}(t) N_{1} k_{110} \quad v-5
$$

As defined previously, $N_{0}=N_{000}$ and $N_{1}=N_{010}$. This expression and certain assumptions were used to obtain an estimate for the value of $k, 10^{\circ}$

If it is assumed, for the moment, that $n_{110}(t)$ has little effect on $n_{100}(t)$, the response of the latter $c$ an be estimated from the computer model (which does not include $n_{110}$ ). This was done and the response was found to be well approximated by

$$
n_{100}(t)=(A-B) e^{-\gamma t}+B \quad V-6
$$

The values of $A, B$, and $r$ were estimated from the code. The results of this esimate for $k 110$ are not sensitive to their exact values. Eq. V-5 was then solved by the same method used for the $n_{002}$ solution in Section III.B.3. This gives

$n_{110}=-\frac{N_{1} B}{N_{0}}\left(1-e^{-N_{0} k t}\right)-\frac{N_{1}(A-B)}{N_{0}\left(1-\gamma / N_{0} k\right)}\left(e^{-\gamma t}-e^{-N_{0} k t}\right) \quad V-7$

with $k=k 110^{\circ}$ This expression has the same behavior as the data. The second term on the right gives rise to a bump which appears superimposed on the monotonic rise of the first term. 
The hot-band signal is a measure of the population difference, $n_{011^{-n}} n_{10}$. Thus the $n_{011}$ behavior is

also needed for this estimate. A reasonable estimate for $n_{011}$ is a rising exponential with a very fast risetime which was determined from the 9-um pump data.

The time of the peak in the data was chosen as the feature to match by adjustment of $k_{110}$. This time was fairly easy to measure ( $375 \mathrm{~ns}$ at 10 torr), and the n110 expression, above, shows the peak time should be sensitive to $k 110^{\circ}$. The resulting estimate obtained was

$$
k_{110}=1.4 \times 10^{6} \sec ^{-1} \operatorname{torr}^{-1} \quad v-8
$$

The corresponding plot of $n_{01}-n_{110}$ is stown in Fig. $v-10$. The shape compares favorably with the data shown in Fig. IV-8. The crosses shown in Fig. V-10 are data points transferred from the scope-trace photo with a scale factor chosen to match the amplitudes at the peaks.

One assumption in the derivation was found not to be very good, however. The calculated density change, $n_{110}$. was a sizeable fraction of $n_{100}$, so the perturbation assumption was probably not reasonable. At this point it was decided that further analysis of this data was beyond the scope of the present study. Further discussion of this result is included in the next chapter. 


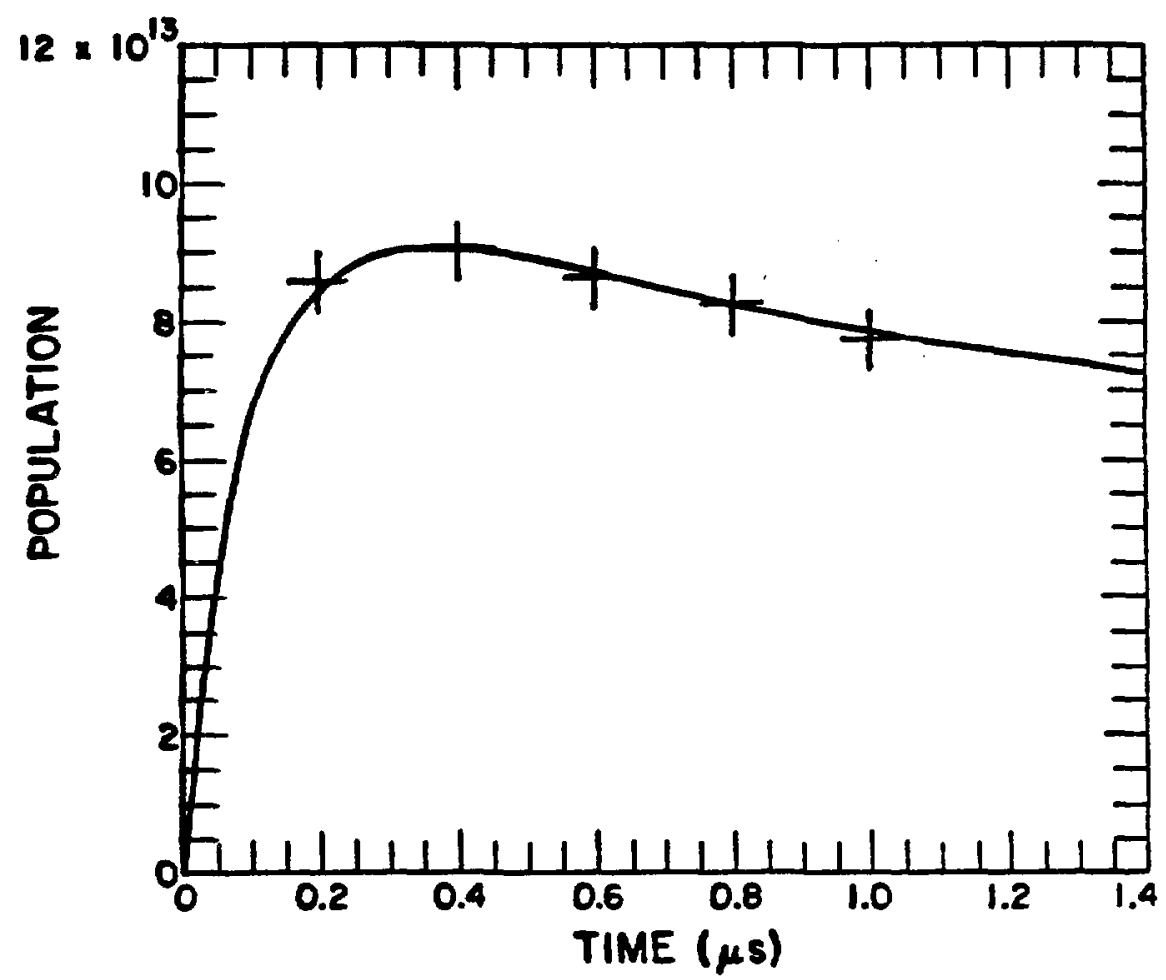

Figure V-10. Plot of the Final $n_{01} y^{-n}{ }_{110}$ Model

The resulting curve from the $n_{01} 1^{-n} 110$ interpretation described in the text is shown. The

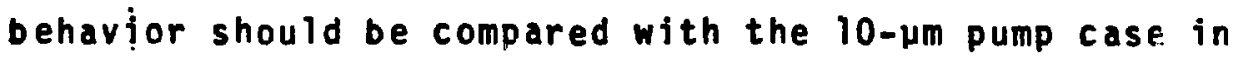
Fig. IV-8. The data points shown have been taken from that figure. 


\section{CHAPTER VI}

\section{DISCUSSION AND FINAL REMARKS}

\section{A. Rotational Energy Transfer}

The pumped level relaxation results have been given in Table V-1. The values show some important aspects of the relaxation relating to temperature dependence, the ground state $V-V$ mechanism, and the dependence on the level which is pumped.

The value of the rotational relaxation rate coefficient found in this study at $700^{\circ} \mathrm{K}$ is essentially the same (within the error bars) as that obtained in the experiments of Jacobs et al. and Cheo and Abrams. In those previous investigations the translational temperature was roughly half that in the present case. This means the rate constant, in torr $\mathrm{sec}^{-1}$, is approximately independent of temperature, thus $k\left(\mathrm{~cm}^{3}\right.$ $\sec ^{-1}$ ) is proportional to $T$. Since $k=\langle\sigma\rangle\langle v\rangle$, the cross section must then be approximately proportional to $T^{+\frac{1}{2}}$.

The importance of temperature dependence in the study of rotational relaxation was discussed in Chapter II.B. If the analysis with only two data points in the 
preceeding paragraph is valid, $\mathrm{CO}_{2}$ is among the molecules for which the rotational transfer probability increases with increasing temperature.

The R-branch probe results which are shown in lines 5 and 6 of the table $(V-1)$ show a constant which is lower than the corresponding P-branch results by about $10 \%$, but the error bars show the difference to be almost insignificant. The implication here is that 100 and 020 relax in essentially the same manner as 001 . This rules out the possibility that the ground state $V-V$ mechanism (Sharma and Kern, 71) is a significant process in 001 relaxation. The same conclusion was reached in the detailed model study of the mechanism (Section III.A.3).

The last point to be made concerning the pumped level experiment relates to lines $1-3$ in Table $V-1$. These indicate there is very little dependence of the relaxation constant on whether $j=9,21$, or 33 is pumped. These levels are below, near, and above the peak of the population probability curve of Fig. II-2. The relaxation is apparently not sensitive to different regions of the rotational distribution, at least within the span of the levels studied.

In addition to the pumped level decay, the $j$-dependence of the rotational relaxation was examined in this experiment. The results are dramatically different 
from what would be expected from the monotonic responses observed in the previous work of Cheo and Abrams.

The population response plots of the neighboring levels are certainly not monotonic in the present work. There is a large overshoot for small $\Delta \mathrm{J}$ transfers, especially for $\Delta J= \pm 2$. This is a feature that cannot be reproduced by reservoir models but is seen in the $\Delta J=2$ model. On the other hand, each of the levels studied has a substantial initial slope. This implies that a cascade process is not responsible for the large $\Delta J$ behavior. Each of these two models seems to oversimplify the true process.

The risetime plots lead to the same conclusion. The reservoir model predicts risetimes which are independent of $\Delta J$, while for the $\Delta J=2$ case, the risetimes would be an extremely strong function of $\Delta \mathrm{J}$. The measured risetimes do not show either of these characteristics. To reproduce the results a model would have to have both substantial cross sections for large $\Delta J$ transfers and enhanced small $\Delta J$ cross sections. The former would be needed to give sizeable initial slopes for large $\Delta J$ responses while the latter would be required to produce the observed nonmonotonic features. The development of such a model is beyond the scope of this study. 
The overshoot which has been observed cannot really be said to be in conflict with the Cheo and Abrams results because the two experiments were performed at very different temperatures. It is possible that the small $\Delta J$ transfer mechanism is strongly temperature dependent although there is no known theoretical basis to support this notion.

It is very interesting that the $\mathrm{He}+\mathrm{CO}_{2}$ model (Preston and Pack, 78) has general features similar to the present $\mathrm{CO}_{2}+\mathrm{CO}_{2}$ data. That model (see Fig. III-3(d))

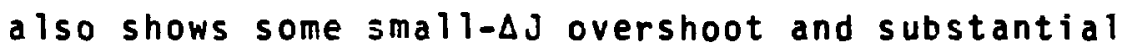
large- $\Delta \mathrm{J}$ transfer. These characteristics are more pronounced in the $\mathrm{CO}_{2}+\mathrm{CO}_{2}$ data, but the $\mathrm{He}+\mathrm{CO}_{2}$ model and the data are, for the most part, very similar.

B. Resonant Vibrational Energy Transfer

In the $V-V$ portion of the experiment resonant $v_{3}$ and $v_{2}$ transfers have been observed at $700^{\circ} \mathrm{K}$ by pumping the 001 level and monitoring the migration of population with sequence-band $(002+101)$ and hot-band $(011+110)$ lasers. The determination of the rate constant $k_{002}$ for the process $001 \rightarrow 002$ is the primary result of the $v_{3}$ study; $k_{10}$ and $k_{01}$ were also determined. The study of resonant $v_{2}$ transfer was not originally part of the experimental plan. However, an 
anomaly in the hot-band data was interpreted as due to the reaction 100+010↔110+000 which, in turn, allowed the determination of the associated rate coefficient, $k_{110}$.

In the $v_{3}$ study a multilevel rate equation model was developed and solved numerically by computer. Study of the resulting solutions showed that a simplified set of rate equations, which cou?d be solved analytically, could model the relevant levels. A least-squares fitting procedure utilizing the analytic solutions was developed to extract the rate constant results from the data. The same rate coefficients have been calculated by Pack at many temperatures including $700^{\circ} \mathrm{K}$. Table VI-l shows the appropriate theoretical values (Pack, 80) and their percent differences from the experimental results. The agreement for $k_{002}$ is fair especially considering the experimental difficulties. The agreement for $k_{011}$ and $k_{101}$ is very good.

These results represent the only experimental information available on the relaxation of the pure $v_{3}$ mode of $\mathrm{CO}_{2}$. Relaxation of the $v_{3}$ mode in $\mathrm{CO}_{2}$ lasers plays an important role in energy storage and extraction. The $k_{002}$ result will certainly contribute to increased understanding of $\mathrm{CO}_{2}$ laser processes.

The fact that the bump feature of the data was seen in the computer model and in the analytic solution leaves 
TABLE VI-1

Coefficient Theory

$K_{011}$

$k_{101}$

$\mathrm{k}_{002}$

1.57

6.38
Experiment

$1.85 \pm .11 \times 10^{6}$

$1.60 \pm .22$

$1.60 \pm .22$
$7.61 \pm .24$

Difference

$1.85 \times 10^{6}$
$7.61 \pm .24$

$-2 \%$

$-16 \%$ 
little doubt that it has been interpreted correctiy. The good reproduction of the crossover time by the fits indicates the value obtained for $k_{002}$ is reliable in spite of the poor signal to noise ratio.

The theoretical calculations of Pack were apparentiy * not completely trusted by him for the $k_{002}$ case. The large value of the computed cross section placed some doubt on the validity of the perturbation methods which were employed. The fair agreement with the measured result indicates the theory is still reasonable beyond the expected limits. The value of $k_{002}$ is remarkably large; it is over half the rotational relaxation constant at $700^{\circ} \mathrm{K}$. At lower temperatures, slightly above room temperature, the theory, if still valid, predicts the $v_{3}$ coefficient would be comparable to k rot.

The $k_{101}$ value is unexpectedly close to the theoretical result in light of its low quality fit. The value previously measured at $295^{\circ} \mathrm{K}, k_{101}=4.2 \pm .3 \times 10^{6}$ (Firizi and Moore, 75 ), and the present result at $700^{\circ} \mathrm{K}$ confirm the theoretically expected temperature dependence (Pack, 80). The present result is thus additional evidence that the energy transfer mechanism is the resonant dipole-dipole interaction.

Previous data, $k_{011}=1.6 \pm .2 \times 10^{6}$ at $700^{\circ} \mathrm{K}$ (Doyennette et al,, 75), and calculations (Pack, 80) existed for $k_{01}$, but it played such an important part 
in the relaxation studied that its confirmation was desirable. The agreement of the present $k_{011}$ result with those previous studies was satisfying and gives support for the accuracy of the $k_{002}$ and $k_{101}$ values.

The hot-band probe observation that 110 population is drained when 100 is depleted by the pump is quite likely due to the resonant process $110+100$. This interpretation has resulted in the estimate for the corresponding rate constant, $k_{110}=1.4 \times 10^{6}$ torr $^{-1} \mathrm{sec}^{-1}$. The $110+100$ process is somewhat analogous to other bending mode relaxation processes (Judd, 75$)$. The present value is larger than, but comparable to, some of the fast rate constants given in that summary.

The $v_{3}$ transition moments are considerably larger than those associated with the bending mode as can be seen in Table II-2. Thus one would expect smaller $v_{2}$-transfer rates. The large value for $k 110$ implies resonant $V-V$ transfer is important in bending mode relaxation also. The data is, in addition, somewhat unique in that it can be interpreted as due to a single bending mode reaction. Thus it should provide a straightforward test of the theory, if the appropriate calculation is ever performed. There are some qualitative aspects of the $110+100$ reaction which may contribute to the large result. First 
both colliding molecules can undergo strong $Q$ transitions, so in effect there are more reaction channels available. Also the $v_{2}$ part of the 110 wavefunction has three vibration quanta which increases the appropriate transition moment. Finally, as in the 011 state, all rotational levels are present in 110, so more resonant energy combinations may be possible.

\section{Final Remarks}

To summarize, double resonance spectroscopy has been used to observe rotational relaxation and $v_{3}$ and $v_{2}$ energy transfer in $\mathrm{CO}_{2}$ at $700^{\circ} \mathrm{K}$. The rotational results indicated enhanced cross sections for collisions involving small changes in the rotational quantum numbers, but large $\Delta J$ transfers were also possible and were important. The $v_{3}$ transfer results for $k_{01}$ and $k_{101}$ confirmed the theoretical predictions. Even for large cross sections, as was the case for $k_{002}$, the theory gave fair predictions. The $v_{2}$ study showed the $110 \rightarrow 100$ rate constant was almost as large as those found for $v_{3}$ transfer.

The technique of double resonance spectroscopy applied to the $\mathrm{CO}_{2}$ molecule has great potential for studying polyatomic collistons and laser processes. The relatively few transitions utilized in the present case have yielded 
important results, but much more information on $\mathrm{CO}_{2}$ collisions should be obtainable.

For instance, in a recent review (Mannucia, 79, p. 62) several $\mathrm{CO}_{2}$ laser transitions are described which would make interesting probes. The transition from $101+011$ at 17 um would have been valuable in the present study. Also the $9.4 \mu \mathrm{m}$ of the sequence band could have been easily incorporated into the study for additional information. The weak 9-4m line of the hot band was also a possibility which would have been useful in the $v_{3}$ study and, even more so, for the investigation of $v_{2}$ transfer.

The relaxation of the lower laser levels of $\mathrm{CO}_{2}$ is not understood on a state-to-state basis so is an area which is ripe for detailed study. It would be possible to do a more complete study now (than in the early 1970's) since the $100 \div 010$ laser at $13.9 \mu \mathrm{m}$ and the $020+010$ laser at $16.1 \mathrm{um}$ are available as probe lasers. These are cascade lasers, and the transitions are reported to lase readily in simple slow-flow dc discharges (Mannucia, 79, p. 84 ).

The pump lasers needed for such experiments could be the same as the $9 \mu \mathrm{m}$ and $10 \mu \mathrm{m}$ used in the present work, although this would require a high temperature sample, again, or an electrical discharge medium. More intriguing possibilities exist, however. It is possible 
with today's technology that pumping from the ground state could be accomplished. For the $v_{3}$ transfer one might find a coincidence among the several $\mathrm{CO}_{2}$ isotopic species which would allow direct 001 pumping via the HF-pumped isotopic- $\mathrm{CO}_{2}$ lasers at $4.3 \mathrm{\mu m}$. These can be fairly high-power devices (Drozdowicz et al., 81). Then the 001-002 process could be studied at lower temperatures. Great simplification in the analysis should then be possible.

Possibilities also exist for ground-state pumping sources in bending mode relaxation studies. If the $03^{1} 0$ level at $5.2 \mu \mathrm{m}$ could be overpopulated, the various probes discussed above could be utilized to monitor the population flow to several other bending mode levels. Coincidences might be found, for accomplishing this, with the high-power co laser. Another, more exotic, possibility would be to frequency double the output of the existing 10-um $\mathrm{CO}_{2}$ pump.

Ground state pumping would not require high-temperature samples, so that parameter would again be available as a variable in the studies. The $001+002$ ccllision process would be interesting to study as a function of temperature, both to confirm an inverse temperature dependence of the cross section and also to determine how the simple theory (Pack, 80) breaks down for the larger cross sections at lower temperatures. 
Temperature dependence might also make a valuable contribution to the study of bending mode relaxation and rotational relaxation. The temperature behavior of bending mode processes could be useful information for determining which theoretical descriptions are more appropriate for the collisions (Judd, 75 ). In rotational transfer it would be very interesting to measure the temperature dependence of the neighboring level relaxation, especially, those for small $\Delta J$ transfers.

As a final point it should be stated again what unique features $\mathrm{CO}_{2}$ has for the double-resonance study of molecular collisions. Theoretically, the molecule is very interesting as it displays polyatomic characteristics, yet its structure and spectroscopy are simple and well understood. Also $\mathrm{CO}_{2}$ has many laser transitions available which makes double resonance spectroscopy highly attractive. The sometimes complicated analysis required to extract rate coefficients and cross sections is the only shortcoming of the technique. An additional practical point is that the $\mathrm{CO}_{2}$ laser is one of the most widely used in research and industry, therefore, support for its study should be strong. The polyatomic collision processes of $\mathrm{CO}_{2}$ should, thus, be among the first to be understood. 


\section{Acknowledgments}

It is a pleasure to express my gratitude to the many people who have helped me accomplish this project. I would like to give special thanks to Russell Pack (Los Alamos National Laboratory) for sharing his knowledge, to Stanley Sobottka (University of Virginia) for making the right things happen, and to Barry Feldman (Los Alamos National Laboratory) for showing me experimental physics. 
Bibliography

1. Alexander, M. H., 1979. Sudden Theories of Rotationally Inelastic LiH-HCl ind LiH-DCl Collisions, J. Chem. Phys. 71:1683-1691.

2. Bernstein, R. B. ed. 1979. Atom-Molecule Collision Theory: A Guide for the Experimentalist, Plenum.

3. Bevington, P.R. 1969. Data Reduction and Error Analysis for the Physical Sciences, McGraw-Hill Book Co.

4. Burak, I., Noter, Y. and Szöke, A. 1973. Vibration-Vibration Energy Transfer in the $v_{3}$ Mode of $\mathrm{CO}_{2}$, IEEE J. Quaritum Electron., QE-9:541-544.

5. Carroll, T. 0. and Marcus, S. 1968. A Direct Measurement of the Rotational Relaxation Time in $\mathrm{CO}_{2}$, Phys. Lett. 27A:590-591.

6. Cheo, P. K. and Abrams, R. L. 1969. Rotational Relaxation Rate of $\mathrm{CO}_{2}$ Laser Levels, Appl. Phys. Lett. 14:47-49.

7. Doyennette, L., Margottin-Maclou, M., Chakroun, A. and Henry, L. 1975. Vibrational Energy Transfer from the $\left(00^{\circ} 1\right)$ Level of ${ }^{14} \mathrm{~N}_{2} \mathrm{O}$ and ${ }^{12} \mathrm{CO}_{2}$ to the $(\mathrm{m}$, $n^{\ell}$, 1) Levels of These Molecules and Their Isotopic Species, J. Chem. Phys. 62:440-447. 
8. Drozdowicz, Z, Rudko, R. O., Linhares, S. J. and Lax, B. 1981. High Gain 4.3-4.5 um Optically Pumped $\mathrm{CO}_{2}$ Lasers, IEEE J. Quantum Electron., QE- $1: 1574-1580$.

9. Feldman, B. J., Fisher, R. A., Pollock, C. R., Simons, S. W. and Tercovich, R. G. 1977. Intense High-Pressure Sequence-Band $\mathrm{CO}_{2}$ Laser, Opt. Lett. $\underline{2}: 16-18$.

10. Finzi, J. and Moore, C. B. 1975. Relaxation of $\mathrm{CO}_{2}$ $\left(10^{\circ} 1\right), \mathrm{CO}_{2}\left(02^{\circ} 1\right)$, and $\mathrm{N}_{2} \mathrm{O}\left(10^{\circ} 1\right)$ Vibrational Levels by Near-Resonant $V \rightarrow V$ Energy Transfer, J. Chem. Phys. $63: 2285-2288$.

11. Gerry, E. J. and Leonard, D. A. 1966. Measurement of $10.6 \mathrm{\mu m} \mathrm{CO} 2$ Laser Transition Probability and Optical Broadening Cross Sections, Appl. Phys. Lett. 8:227-229. 12. Heil, T. G., Green, S. and Kouri, D. J. 1968. Coupled State Approximations for Scattering of Two Diatomics. J. Chem. Phys. 68:2562-2583.

13. Jackson, J. D. 1962. Classical Electrodynamics, John Wiley and Sons, Inc.

14. Jacobs, R. R., Pettipiece, K. J. and Thomas, S. J. 1974a. Rotational Relaxation Constants for $\mathrm{CO}_{2}$, App 1. Phys. Lett. 24: 375-377. 
15. Jacobs, R. R., Thomas, S. J. and Pettipiece, K. J. 1974b. J-Dependence of Rotational Relaxation in the $\mathrm{CO}_{2} \mathrm{OO}^{\circ} 1$ Vibrational Level, IEEE J. Quantum Electron. QE-10:480-486.

16. Judd, 0. P. 1975. On the Fermi Resonance Levels in $\mathrm{CO}_{2}$, Los Alamos National Laboratory Internal Report LA-5892-MS.

17. Lambert, J. D. 1977. Vibrational and Rotational Relaxation in Gases, Clarendon Press.

18. Mahan, B. H. 1967. Resonant Transfer of Vibrational Energy in Molecular Collisions, J. Chem. Phys. 46:98-101.

19. Manuccia, T. 1979. Molecular Lasers, in Methods of Experimental Physics, Vol. 15B, Academic Press. 20. Massey, H. S. H. 1971. Electronic and Ionic Impact Phenomena, Vol. III. Oxford University Press.

21. Mclellan, E. J. and Figueira, J. F. 1979. Ultrafast Pockels Cells for the Infrared, Rev. Sci. Instrum. 50: $1213-1217$.

22. Moore, C. B. 1973. Vibration-Vibration Energy Transfer, Adv. Chem. Phys. 23:41-83.

23. Murphy, G. M. 1960. Ordinary Differential Equations and Their Solution, D. Van Nostrand Co., Inc. 
24. Pack, R. T. 1980. Analytic Estimation of Almost Resonant holecular Energy Transfer Due to Multipolar Potentials. VV Processes Involving $\mathrm{CO}_{2}, \mathrm{~J}$. Chem. Phys. 72:6140-6152.

25. Preston, R. K. and Pack, R. T. 1978. Mechanism and Rates of Rotational Relaxation of $\mathrm{CO}_{2}(001)$ in He and Ar, J. Chem. Phys. 69:2823-2832.

26. Reid, J. and Siemsen, K. 1977. Laser Power and Gain Measurements on the Sequence Bands of $\mathrm{CO}_{2}, J$. Appl. Phys. 48:2712-2717.

27. Reid, J. and Siemsen, K. 1978. Gain of High-Pressure $\mathrm{CO}_{2}$ Lasers, IEEE J. Quantum Electron., QE-14:217-219. 28. Rose, M. E. 1957. Elementary Theory of Angular Momentum, John Wiley and Sons, Inc.

29. Rothman, L. S. and Benedict, W. S. 1978. Infrared Energy Levels and Intensities of Carbon Dioxide, Appl. Optics, 17:2605-2611.

30. Schiff, L. I. 1968. Quantum Mechanics, McGraw-Hill Book Co.

31. Sharma, R. D. and Brau, C. A. 1967. Near-Resonant Vibrational Energy Transfer in $\mathrm{N}_{2}-\mathrm{CO}_{2}$ Mixtures, Phys. Rev. Lett. 19:1273-1275. 
32. Sharma, R. D. and Brau, C. A. 1969. Energy Transfer in Near-Resonant Molecular Collisions Due to Long-Range Forces with Application to Transfer of Vibrational Energy from $v_{3}$ Mode of $\mathrm{CO}_{2}$ to $\mathrm{N}_{2}$, J. Chem. Phys. 50:924-930.

33. Sharma, R. D. and Kern, C.W. 1971. Theoretical Model for the Differential Quenching Rates of CO Fluorescence by Ortho- and Para-hydrogen, J. Chem. Phys. 55:1171-1188.

34. Smith, K. and Thomson, R. M. 1978. Computer Modeling of Gas Lasers, Plenum, New York.

35. Statz, H., Tang, C. L. and Koster, G. F. 1966. Transition Probabilities Between Laser States in Carbon Dioxide, J. Appl. Phys . 37:4278-4284. 36. Steinfeld, J. I. 1978. Laser and Coherence Spectroscopy, Plenum Press.

37. Stephenson, J. C. and Moore, C. B. 1972. Temperature Dependence of Nearly Resonant Vibration $\rightarrow$ Vibration Energy Transfer in $\mathrm{CO}_{2}$ Mixtures, J. Chem. Phys. 56:1295-1308.

38. Van Kranendonk, 3. 1963. Calculation of the Self-Broadening of Raman Lines Due to Dipolar and Quadrupolar Forces, Can. J. Phys. 41: 433-449. 
39. Whitford, B. G., Siemsen, K. J. and Reid, J. 1977. Heterodyne Frequency Measurements of $\mathrm{CO}_{2}$ Laser Hot-Band Transitions, Opt. Commun. 22:261-264.

40. Yariv, A. 1975. Quantum Electronics, John Wiley and Sons, Inc. 